\section{$\begin{array}{llll}\text { I } & \mathbf{F} & \mathbf{I} & \mathbf{G}\end{array}$ R E S E A R C H $\begin{array}{llllll}\mathbf{R} & \text { E } & \mathrm{P} & \mathrm{O} & \mathrm{R} & \mathrm{T}\end{array}$}

INSTITUT FÜR INFORMATIK

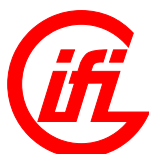

\title{
Existential SECOND-ORDER LOGIC OVER STRINGS
}

Thomas Eiter Georg Gottlob Yuri Gurevich

IFIG RESEARCH REPORT 9702

DECEMBER 1997

Institut für Informatik

JLU Gießen

Arndtstraße 2

D-35392 Giessen, Germany

Tel: $\quad+49-641-99-32141$

Fax: +49-641-99-32149

mail@informatik.uni-giessen.de

www.informatik.uni-giessen.de

JUSTUS-LIEBIG-

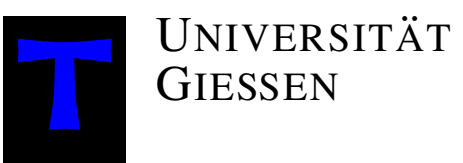



IFIG RESEARCH REPORT

IFIG RESEARCH REPORT 9702, DECEMBER 1997

\title{
EXISTENTIAL SECOND-ORDER LOGIC OVER STRINGS
}

\author{
- MODIFIED MARCH 1998 -
}

\author{
Thomas Eiter ${ }^{1} \quad$ Georg Gottlob $^{2} \quad$ Yuri Gurevich $^{3}$
}

\begin{abstract}
Existential second-order logic (ESO) and monadic second-order logic (MSO) have attracted much interest in logic and computer science. ESO is a much more expressive logic over word structures than MSO. However, little was known about the relationship between MSO and syntactic fragments of ESO. We shed light on this issue by completely characterizing this relationship for the prefix classes of ESO over strings, (i.e., finite word structures). Moreover, we determine the complexity of model checking over strings, for all ESO-prefix classes. Let $\operatorname{ESO}(\mathcal{Q})$ denote the prefix class containing all sentences of the shape $\exists \mathbf{R} Q \varphi$ where $\mathbf{R}$ is a list of predicate variables, $Q$ is a first-order quantifier prefix from the prefix set $\mathcal{Q}$, and $\varphi$ is quantifier free. We show that $\operatorname{ESO}\left(\exists^{*} \forall \exists^{*}\right)$ and $\operatorname{ESO}\left(\exists^{*} \forall \forall\right)$ are the maximal standard ESO-prefix classes contained in MSO, thus expressing only regular languages. We further prove the following dichotomy theorem: An ESO prefix-class either expresses only regular languages (and is thus in MSO), or it expresses some NP-complete languages. We also give a precise characterization of those ESO-prefix classes which are equivalent to MSO over strings, and of the ESO-prefix classes which are closed under complementation on strings.
\end{abstract}

\footnotetext{
${ }^{1}$ Institut für Informatik, Universität Gießen, Arndtstraße 2, D-35392 Gießen, Germany. Email: eiter@informatik.uni-giessen.de

${ }^{2}$ Institut für Informationssysteme, Technische Universität Wien, Paniglgasse 16, A-1040 Wien, Austria. E-mail: gottlob@dbai.tuwien.ac.at

${ }^{3}$ Electrical Engineering and CS Department, University of Michigan, Ann Arbor, MI 48109-2122, USA. Email: gurevich@eecs.umich.edu
}

An extended abstract of this paper will appear in the Proceedings of the 13th IEEE Symposium on Logic in Computer Science (LICS '98), June 21-24, Indianapolis, Indiana, 1998.

Copyright (c) 1998 by the authors 


\section{Introduction}

Second-order logic over finite structures has attracted the interest of logicians, mathematicians, and computer scientists for a long time. In particular, several important results have been obtained which link logic to automata theory and complexity theory.

Two fundamental results in this context are the famous Büchi Theorem [7], which says that monadic second-order logic (MSO) over strings precisely characterizes the regular languages, and Fagin's Theorem [14], which states that existential second-order logic (ESO) exactly expresses the NP properties over finite structures (in particular, over finite strings). Thus, over strings, ESO is a much more expressive logic than MSO. However, little was known about the relationship between syntactic fragments of ESO and MSO.

In this paper we shed light on this issue by investigating prefix classes of (nonmonadic) ESO over finite strings. Before explaining in more detail both the problem studied and the obtained results, we spend a few paragraphs describing the context of our research and the most important earlier results related to it.

Logical Characterization of NP. The class NP is one of the most well-known classes of problems, and has been attracting much interest from both the practical as well as the theoretical side. To date, a large and steadily increasing number of problems in practice is known to be complete for this class, while no polynomial time algorithm for any of these problems is known; the $\mathrm{P}=\mathrm{NP}$ question is one of the main challenging open problem in computer science.

This question has been tackled from different directions in the hope of utilizing tools and the rich body of knowledge from different, well-developed areas. One such attempt was a reduction of the $\mathrm{P}=\mathrm{NP}$ question to problems in logic. In this context, Fagin [14] gave a purely logical characterization of NP in terms of second-order logic, where there is no notion of machine, computation, or time. He proved that over finite structures, the properties which are decidable in NP are precisely those which are definable in existential second-order logic (ESO), i.e., expressible through a sentence of the form $\exists \mathbf{R} \varphi$, where $\exists \mathbf{R}$ means existential quantification over a list $\mathbf{R}=R_{1}, \ldots, R_{n}$ of relational variables $R_{i}$ and $\varphi$ is a first-order formula.

Fagin's Theorem was successfully used in various areas for establishing different types of results. For example, it has been exploited in database theory for assessing the expressive power of query languages, cf. [28, 45, 43], or in computation theory to characterize subclasses of NP or establish logically defined hierarchies of nondeterministic complexity classes within NP [2, 19, 33, 35]. Another use is in the area of optimization theory, where based on Fagin's Theorem, logical definitions of optimization problems were given, cf. [38, 37, 29].

Prefix Classes. In the above investigations, syntactic subclasses of ESO were studied. In particular, prefix classes play an important role. Prefix classes are the most natural and the most commonly studied fragments of predicate logic. A prefix class is a class of formulas in prenex normal whose quantifier prefixes obey a certain pattern. Denote by $\operatorname{ESO}(\mathcal{Q})$ the prefix class consisting of all ESO-sentences $\exists \mathbf{R} \varphi$, where $\varphi$ is in prenex normal form with a quantifier prefix from a first-order prefix class $\mathcal{Q}$. Then, Fagin's Theorem actually characterizes NP as the class $\operatorname{ESO}\left(\forall^{*} \exists^{*}\right)$.

The interest in prefix-classes dates back a long time ago. Actually, the classical decision problem of Hilbert is the following problem, where $\operatorname{FO}(\mathcal{Q})$ denotes the set of prenex first-order formulas, possibly containing free occurrences of predicate variables: ${ }^{1}$

\footnotetext{
${ }^{1}$ Note that in the context of model checking, the only free predicate variables of a FO formula may be the predicate symbols in
} 
Instance: formula $\varphi$ in $\mathrm{FO}(\mathcal{Q})$

Question: Is $\varphi$ (finitely) satisfiable?

Reformulated in the context of second-order logic, this is equivalent to whether for a given $\mathcal{Q}$ :

Instance: formula $\varphi$ in $\operatorname{ESO}(\mathcal{Q})$

Question: Is $\varphi$ (finitely) satisfiable?

This question has been studied in depth over the past decades, and an exhaustive classification of decidable and undecidable prefix classes is known (see [5]); there are huge complexity gaps between elementarily decidable and undecidable classes. The $\operatorname{ESO}(\mathcal{Q})$ classification played an important role in the identification of fragments of ESO which obey the 0-1 law, i.e., the property that over finite structures, a sentence is almost surely true or almost surely false, cf. $[30,36]$. It turned out that there is a close relationship between decidable classes and those satisfying the 0-1 law.

Ordered Structures, Strings, and Büchi's Theorem. While the above results are on arbitrary (finite) structures, computer science mainly deals with ordered structures. In fact, the input to a computing device such as a finite automaton or a Turing machine is implicitly ordered by the position of the data in the input stream or on the input tape, respectively. Accordingly, many important issues in finite model theory were considered in the context of ordered structures (see below for a short description of results and references).

Strings, i.e., words over a finite alphabet, are ordered structures of particular importance. The set of strings satisfying a given formula $\Phi$ is a formal language. We can thus directly compare classes of formal languages to logical formalisms.

In order to do this formally, we need to confine to a logical representation of strings. There are several possibilities and we have chosen the simplest here. We represent a string over an alphabet $A$ as a structure over a finite universe $U=\{1, \ldots, n\}$ (representing the positions of the string), equipped with the natural successor relation Succ over $U$, constants $\min$ and $\max$ for the first and the last position, respectively, and a predicate $C_{a}$ for each letter $a \in A$, such that $C_{a}(i)$ is true iff the $i$-th position of the string consists of letter $a$.

A fundamental result relating logic to formal languages is Büchi's Theorem [7] (also found by Trakhtenbrot [53]), which says that monadic second-order logic (MSO) expresses over finite strings precisely the regular languages. Hence, over finite strings, MSO is much weaker than ESO, which expresses all languages in NP.

In fact, over finite strings, even the class $\operatorname{ESO}\left(\forall^{*}\right)$ expresses all languages in NP; this follows from the more general result that over finite successor structures, i.e., finite structures equipped with a successor predicate, every $\operatorname{ESO}$ sentence is equivalent to some $\operatorname{ESO}\left(\forall^{*}\right)$ sentence $[32,13]$.

Main Problems Studied. Combining and extending the results of Büchi and Fagin, it is natural to ask: What about (nonmonadic) prefix classes $\operatorname{ESO}(\mathcal{Q})$ over finite strings? We know by Fagin's theorem that all these classes describe languages in NP. But there is a large spectrum of languages contained in NP ranging from regular languages (at the bottom) to NP-hard languages at the top. What can be said about the languages expressed by a given prefix class $\operatorname{ESO}(\mathcal{Q})$ ? Can the expressive power of these fragments be characterized? In order to clarify these issues, we investigated, in particular, the following problems:

the signature of the input structure. 
- Which classes $\operatorname{ESO}(\mathcal{Q})$ express only regular languages?

In other terms, for which fragments $\operatorname{ESO}(\mathcal{Q})$ is it true that for any formula $\Phi \in \operatorname{ESO}(\mathcal{Q})$ the set $\operatorname{Mod}(\Phi)=\{W \mid W \models \Phi\}$ of all finite strings (over a given finite alphabet) satisfying $\varphi$ constitutes a regular language? Any fragment fulfilling this condition is called regular. By Büchi's Theorem this question is identical to the following: Which prefix classes of ESO are (semantically) included in MSO?

Note that by Gurevich's classifiability theorem (cf. [5]) and by elementary closure properties of regular languages, it follows that there is a finite number of maximal regular prefix classes $\operatorname{ESO}(\mathcal{Q})$, and similarly, of minimal nonregular prefix classes; the latter are, moreover, standard, i.e., the quantifier prefix class $\mathcal{Q}$ is either the set of all prefixes or it can be described by a string over the alphabet $\left\{\forall, \exists, \forall^{*}, \exists^{*}\right\}$. It is our aim to determine the maximal regular prefix classes and the minimal nonregular prefix classes.

- What is the complexity of model checking (over strings) for the nonregular classes $\operatorname{ESO}(\mathcal{Q})$ ?

Model checking for regular classes $\operatorname{ESO}(\mathcal{Q})$ is easy: it is feasible by a finite state automaton. We also know (e.g. by Fagin's Theorem) that some classes $\operatorname{ESO}(\mathcal{Q})$ allow us to express NP-complete languages. It is therefore important to know (i) which classes $\operatorname{ESO}(\mathcal{Q})$ can express NP-complete languages, and (ii) whether there are prefix classes $\operatorname{ESO}(\mathcal{Q})$ of intermediate complexity between regular and NP-complete classes.

- Which classes $\operatorname{ESO}(\mathcal{Q})$ capture the class REG of all regular languages? A class of logical sentences captures REG, if all regular languages and only those can be expressed in it. By Büchi's Theorem, this question is equivalent to the question of which classes $\operatorname{ESO}(\mathcal{Q})$ have exactly the expressive power of MSO over strings.

- For which classes $\operatorname{ESO}(\mathcal{Q})$ is finite satisfiability decidable, i.e., given a formula $\Phi \in \operatorname{ESO}(\mathcal{Q})$, decide whether $\Phi$ is true on some finite string ?

- Which classes $\operatorname{ESO}(\mathcal{Q})$ are closed under complementation over strings?

Main Results. The present paper answers all the above questions exhaustively. Some of our results are rather unexpected. In particular, we prove a surprising dichotomy theorem which sharply classifies all $\operatorname{ESO}(\mathcal{Q})$ classes as either regular or intractable. Our main results are summarized as follows.

(1) The class $\operatorname{ESO}\left(\exists^{*} \forall \exists^{*}\right)$ is regular (Theorem 7.1). This theorem constitutes the technically most involved result of this paper. Given that this class is nonmonadic, it was not possible to exploit any of the ideas underlying Büchi's proof for proving it regular. The main difficulty consists in the fact that relations of higher arity may connect elements of a string that may be very distant from one another and it is not $a$ priori clear how a finite state automaton should be able to guess such connections and check their global consistency. To solve this problem, we had to develop completely new methods. In particular, we proved new combinatorial results on hypergraphs and applied them to logic.

Interestingly, model checking for the fragment $\operatorname{ESO}\left(\exists^{*} \forall \exists^{*}\right)$ is NP-complete over graphs. For example, the well-known set-splitting problem can be expressed in it. Thus the fact that our input structures are monadic strings is essential (just as for MSO).

(2) The class $\operatorname{ESO}\left(\exists^{*} \forall \forall\right)$ is regular (Theorem 8.1). The regularity proof for this fragment is easier but also required new techniques. Note that model checking for this class, too, is NP-complete over graphs.

(3) Any class $\operatorname{ESO}(\mathcal{Q})$ not contained in the union of $\operatorname{ESO}\left(\exists^{*} \forall \exists^{*}\right)$ and $\operatorname{ESO}\left(\exists^{*} \forall \forall\right)$ is not regular (Proposition 3.1). 
Thus $\operatorname{ESO}\left(\exists^{*} \forall \exists^{*}\right)$ and $\operatorname{ESO}\left(\exists^{*} \forall \forall\right)$ are the maximal regular standard prefix classes. The unique maximal (general) regular ESO-prefix class is the union of these two classes, i.e, $\operatorname{ESO}\left(\exists^{*} \forall \exists^{*}\right) \cup \operatorname{ESO}\left(\exists^{*} \forall \forall\right)=$ $\operatorname{ESO}\left(\exists^{*} \forall\left(\forall \cup \exists^{*}\right)\right)$ (Theorem 8.7).

It turns out that there are three minimal nonregular ESO-prefix classes, namely the standard prefix classes $\operatorname{ESO}(\forall \forall \forall), \operatorname{ESO}(\forall \forall \exists)$, and $\operatorname{ESO}(\forall \exists \forall)$. All these classes express nonregular languages by formulas whose list of second-order variables consists of a single binary predicate variable.

Therefore, results 1.-3. give a complete characterization of the regular classes $\operatorname{ESO}(\mathcal{Q})$. A picture of the situation is given in Figure 1. The picture also visualizes further results, and is explained in more detail below.

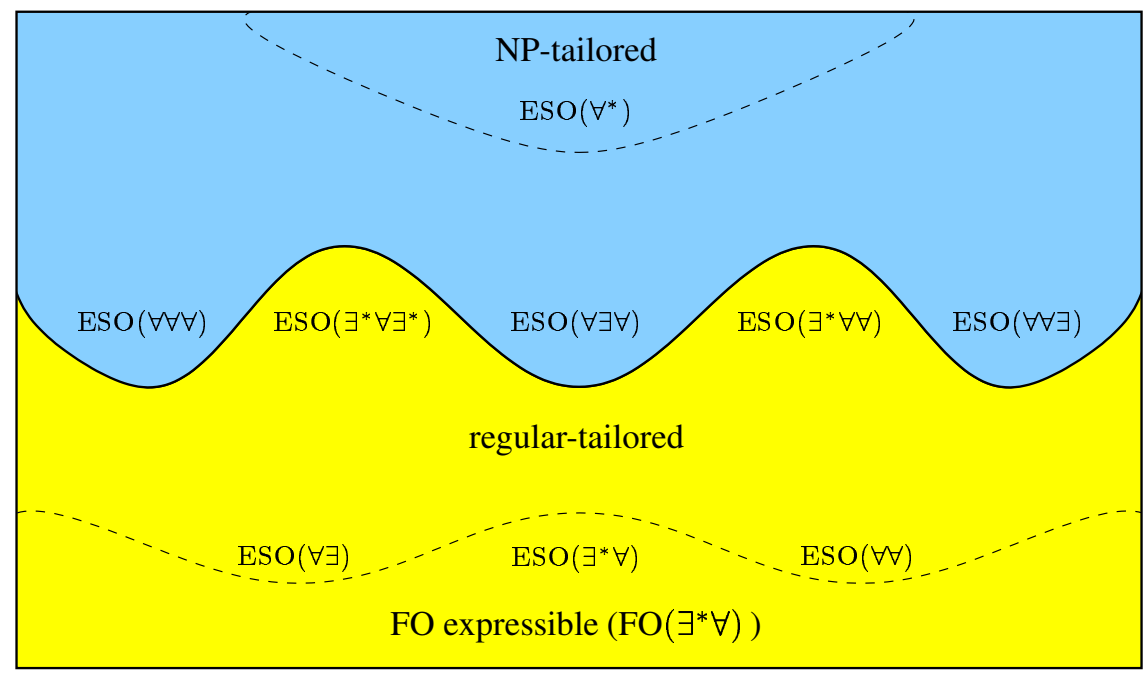

regular $\quad \square$ NP-hard

Figure 1: Complete picture of the ESO-prefix classes on finite strings

(4) We obtain the following dichotomy theorem: Let $\operatorname{ESO}(\mathcal{Q})$ be any prefix class. Then, either $\operatorname{ESO}(\mathcal{Q})$ is regular, or $\operatorname{ESO}(\mathcal{Q})$ expresses some NP-complete language (Theorem 9.3). This means that model checking for $\operatorname{ESO}(\mathcal{Q})$ is either possible by a deterministic finite automaton (and thus in constant space and linear time) or it is already NP-complete. Moreover, for all NP-complete classes $\operatorname{ESO}(\mathcal{Q})$, NP-hardness holds already for sentences whose list of second-order variables consists of a single binary predicate variable. There are no fragments of intermediate difficulty between REG and NP.

(5) The above dichotomy theorem is paralleled by the solvability of the finite satisfiability problem for ESO (and thus FO) over strings. We show that over finite strings, satisfiability of a given formula from a class $\operatorname{ESO}(\mathcal{Q})$ is decidable if and only if $\operatorname{ESO}(\mathcal{Q})$ is regular (Theorem 10.1).

(6) We give a precise characterization of those prefix classes of ESO which are equivalent to MSO over strings, i.e. of those prefix fragments that capture the class REG of regular languages (Theorem 11.4). 
This provides us with completely new logical characterizations of REG. Moreover, we establish that any regular ESO-prefix class is over strings either equivalent to full MSO, or is contained in first-order logic, in fact, in $\mathrm{FO}\left(\exists^{*} \forall\right)$ (Theorem 11.3). We further show that there is a unique minimal ESO prefix class which captures NP, namely $\operatorname{ESO}\left(\forall^{*}\right)$ (Proposition 9.1). Our proof uses results in $[32,13]$ and well-known hierarchy theorems.

(7) We give a precise characterization of those regular prefix classes of ESO which, over strings, are closed under complementation. In particular, we show that any nontrivial regular class $\operatorname{ESO}(\mathcal{Q})$ is closed under complementation iff some quantifier prefix $Q \in \mathcal{Q}$ contains either the sequence $\forall \forall$ or the sequence $\forall \exists$; this is the case iff $\operatorname{ESO}(\mathcal{Q})$ captures REG (Theorem 11.4). Moreover, it follows from our previously described results (1.-4.) that if NP $\neq$ co-NP, then no nonregular prefix class is closed under complementation. Assuming NP $\neq$ co-NP we have thus completely determined those prefix classes of ESO which are closed under complementation over strings (Theorem 11.6).

Our main results are summarized in Figure 1. In this figure, the set of all ESO-prefix classes is divided into four regions. The upper two regions contain all classes that express nonregular languages, and therefore, as we show, also NP-complete languages. The uppermost region contains those classes which capture NP, i.e., express all languages in NP. These classes are called NP-tailored. The embracing region, separated by a dashed line, are those classes which can express some NP-hard languages, but not all languages in NP. Its bottom is constituted by the minimal nonregular classes, $\operatorname{ESO}(\forall \forall \forall), \operatorname{ESO}(\forall \exists \forall)$, and $\operatorname{ESO}(\forall \forall \exists)$. The lower two regions contain all regular classes. The maximal regular standard prefix classes are $\operatorname{ESO}\left(\exists^{*} \forall \exists^{*}\right)$ and $\operatorname{ESO}\left(\exists^{*} \forall \forall\right)$. The dashed line separates the classes which capture precisely REG(called regular-tailored), from those which do not; by our results, the expressive capability of the latter classes is restricted to firstorder logic (in fact, to $\mathrm{FO}\left(\exists^{*} \forall\right)$ ). The minimal classes which capture REG are $\operatorname{ESO}(\forall \exists)$ and $\operatorname{ESO}(\forall \forall)$.

Furthermore, all nontrivial classes contained in the lowest region are (provably) not closed under complementation. All classes in the upper region of regular classes (regular-tailored) are closed under complementation, while those in the region above regular classes are not closed under complementation unless $\mathrm{NP}=$ co-NP.

Potential Applications. Monadic second-order logic over strings is currently used in the verification of hardware, software, and distributed systems. An example of a specific tool for checking specifications based on MSO is the MONA tool developed at the BRICS research lab in Denmark [3, 21, 26].

Observe that certain interesting desired properties of systems are most naturally formulated in nonmonadic second-order logic. Consider, as an unpretentious example ${ }^{2}$, the following property of a ring $P$ of processors of different types, where two types may either be compatible or incompatible with each other. We call $P$ tolerant, if for each processor $p$ in $P$ there exist two other distinct processors $\operatorname{backup}_{1}(p) \in P$ and backup $_{2}(p) \in P$, both compatible to $p$, such that the following conditions are satisfied:

1. for each $p \in P$ and for each $i \in\{1,2\}, \operatorname{backup}_{i}(p)$ is not a neighbor of $p$;

2. for each $i, j \in\{1,2\}$, backup $_{i}\left(\operatorname{backup}_{j}(p)\right) \notin\left\{p, \operatorname{backup}_{1}(p), \operatorname{backup}_{2}(p)\right\}$.

Intuitively, we may imagine that in case $p$ breaks down, the workload of $p$ can be reassigned to $\operatorname{backup}_{1}(p)$ or to $\operatorname{backup}_{2}(p)$. Condition 1 reflects the intuition that if some processor is damaged, there is some likelihood

\footnotetext{
${ }^{2}$ Our goal here is merely to give the reader some intuition about a possible type of application.
} 
that also its neighbors are (e.g. in case of physical affection such as radiation), thus neighbors should not be used as backup processors. Condition 2 states that the backup processor assignment is antisymmetric and anti-triangular; this ensures, in particular, that the system remains functional, even if two processors of the same type are broken (further processors of incompatible type might be broken, provided that broken processors can be simply bypassed for communication).

Let $T$ be a fixed set of processor types. We represent a ring of $n$ processors numbered from 1 to $n$ where processor $i$ is adjacent to processor $i+1(\bmod n)$ as a string of length $n$ from $T^{*}$ whose $i$-th position is $\tau$ if the type of the $i$-th processor is $\tau$; logically, $C_{\tau}(i)$ is then true. The property of $P$ being tolerant is expressed by the following second order sentence $\Phi$ :

$$
\begin{aligned}
\Phi: \quad \exists R_{1}, R_{2}, \forall x \exists y_{1}, y_{2} . & \operatorname{compat}\left(x, y_{1}\right) \wedge \operatorname{compat}\left(x, y_{2}\right) \wedge \\
& R_{1}\left(x, y_{1}\right) \wedge R_{2}\left(x, y_{2}\right) \wedge \\
& \bigwedge_{i=1,2} \wedge \wedge_{j=1,2}\left(\neg R_{i}\left(y_{j}, x\right) \wedge \neg R_{1}\left(y_{j}, y_{i}\right) \wedge \neg R_{2}\left(y_{j}, y_{i}\right)\right) \wedge \\
& x \neq y_{1} \wedge x \neq y_{2} \wedge y_{1} \neq y_{2} \wedge \\
& \neg \operatorname{Succ}\left(x, y_{1}\right) \wedge \neg S u c c\left(y_{1}, x\right) \wedge \neg \operatorname{Succ}\left(x, y_{2}\right) \wedge \neg \operatorname{Succ}\left(y_{2}, x\right) \wedge \\
& \left((x=\max ) \rightarrow\left(y_{1} \neq \min \wedge y_{2} \neq \min \right)\right) \wedge \\
& \left((x=\min ) \rightarrow\left(y_{1} \neq \max \wedge y_{2} \neq \max \right)\right),
\end{aligned}
$$

where compat $(x, y)$ is the abbreviation for the formal statement that processor $x$ is compatible to processor $y$ (which can be encoded as a simple boolean formula over $C_{\tau}$ atoms).

$\Phi$ is the natural second-order formulation of the tolerance property of a ring of processors. This formula is in the fragment $\operatorname{ESO}\left(\exists^{*} \forall \exists^{*}\right)$; hence, by our results, we can immediately classify tolerance as a regular property, i.e., a property that can be checked by a finite automaton.

In a similar way, one can exhibit examples of $\operatorname{ESO}\left(\exists^{*} \forall \forall\right)$ formulas that naturally express interesting properties whose regularity is not completely obvious a priori. We thus hope that our results may find applications in the field of computer aided verification.

Further Related Work. Since Büchi's logical characterization of the regular languages and Fagin's logical characterization of NP, several further logical characterizations of complexity classes or types of formal languages have been obtained.

The following are some classical results concerning general (not necessarily monadic) finite structures. Stockmeyer [48] has shown that full second-order logic captures the polynomial hierarchy (PH). Immerman [23] and Vardi [54] proved that polynomial time is captured by fixpoint logic over ordered structures, and Grädel $[17,18]$ established this for $\operatorname{ESO}\left(\forall^{*}\right.$, Horn $)$. The related result that the well-known database query language Datalog captures $\mathrm{P}$ over ordered structures is already implicit in [54, 23]. Abiteboul and Vianu have studied several other database query languages, and they showed that the classes of total and partial fixpoint queries coincide on arbitrary finite structures if and only if P = PSPACE (see [1]). Many complexity classes, including LOGSPACE and NLOGSPACE, were logically characterized by Immerman [23]. Most of these results and many others are covered by the books or surveys [25, 10, 20, 16].

Our results add to previous knowledge about the relationships between nonmonadic ESO fragments and MSO over strings. They contrast with previous results on graphs. We show that existential MSO and $\operatorname{ESO}\left(\exists^{*} \forall \exists^{*}\right)$ coincide over strings. This is not true for graphs. It was known that over finite graphs, disconnectivity is expressible in existential MSO [15], and 2-colorability or completeness of a graph are clearly 
in existential MSO; however, none of these properties is expressible in $\operatorname{ESO}\left(\exists^{*} \forall \exists^{*}\right)$, even in presence of a successor [12]. Therefore, $\operatorname{ESO}\left(\exists^{*} \forall \exists^{*}\right)$ and MSO have different expressive power over ordered graphs. Further relevant work on discussing ESO and MSO fragments over graphs and general structures can be found in $[8,46,47,42,9]$.

To our best knowledge, there has been no previous characterization of the regular languages by nonmonadic fragments of ESO. However, many papers cover either extensions or restrictions of MSO or REG.

Lynch [33], for example, has studied the logic over strings obtained from existential MSO by adding addition. He proved that model checking for this logic lies in $\operatorname{NTIME}(n)$, i.e., in nondeterministic linear time. Grandjean and Olive [19, 35] obtained interesting results related to those of Lynch. They gave logical representations of the class NLIN, i.e., linear time on random access machines, in terms of second-order logic with unary functions instead of relations (in their setting, also the input string is represented by a function).

Lautemann, Schwentick and Thérien [31] recently proved that the class CFL of context-free languages is characterized by ESO formulas of the form $\exists B \varphi$ where $\varphi$ is first-order, $B$ is a binary predicate symbol, and the range of the second-order quantifier is restricted to the class of matchings, i.e., pairing relations without crossover. Note that this is not a purely prefix-syntactic characterization of CFL. From our results and the fact that some languages which are not context-free can be expressed in the minimal nonregular ESO-prefix classes, it follows that a syntactic characterization of CFL by means of ESO-prefix classes is impossible.

Several restricted versions of REG where studied and logically characterized by restricted versions of ESO. McNaughton and Papert [34] showed that first-order logic with a linear ordering precisely characterizes the star-free regular languages. This theorem was extended by Thomas [52] to $\omega$-languages, i.e., languages with infinite words. Later several hierarchies of the star-free languages were studied and logically characterized (see, e.g. [52, 39, 40, 41]). Straubing, Thérien and Thomas [50] showed that firstorder logic with modular counting quantifiers characterize the regular languages whose syntactic monoids contain only solvable groups. These and many other related results can be found in the books and surveys $[49,52,39,40,41]$.

Structure of the paper. The rest of this paper is organized as follows. Section 2 introduces basic concepts and notation. In Section 3, we show that the classes $\operatorname{ESO}(\forall \forall \forall), \operatorname{ESO}(\forall \forall \exists)$, and $\operatorname{ESO}(\forall \exists \forall)$ all express the canonical nonregular language $L=\left\{a^{n} b^{n}\right\}$. In Section 4, we derive a new combinatorial theorem on hypergraphs, which is a crucial tool for proving that $\operatorname{ESO}\left(\exists^{*} \forall \exists^{*}\right)$ is regular. The latter result, which is the technically most demanding of this paper, is gradually established in Sections 5-7. In particular, Section 5 proves the result under the restriction to successor-free $\operatorname{ESO}\left(\forall \exists^{*}\right)$ sentences; Section 6 generalizes it to $\operatorname{ESO}\left(\forall \exists^{*}\right)$ sentences. The general result is then proved in Section 7 . The regularity of the class $\operatorname{ESO}\left(\exists^{*} \forall \forall\right)$ is shown in Section 8. For better readability, the proof in this section is given under simplifying assumptions; a full proof is in the appendix.

The problem of model checking is considered in Section 9, where we prove a dichotomy theorem for model checking. In Section 10, we determine the classes $\mathcal{Q}$ for which finite satisfiability is decidable. Section 11 identifies those classes $\operatorname{ESO}(\mathcal{Q})$ which capture REG and those which are closed under complementation. The final Section 12 addresses further research issues and concludes the paper. 


\section{Preliminaries and Notation}

We consider second-order logic with equality (unless otherwise stated explicitly) and without function symbols of positive arity. Predicates are denoted by capitals and individual variables by lower case letters; a bold face version of a letter denotes a tuple of corresponding symbols.

A prefix is any string over the alphabet $\{\exists, \forall\}$, and a prefix set is any language $\mathcal{Q} \subseteq\{\exists, \forall\}^{*}$ of prefixes. A prefix set $\mathcal{Q}$ is trivial, if $\mathcal{Q}=\emptyset$ or $\mathcal{Q}=\{\lambda\}$, i.e., it consists of the empty prefix. In the rest of this paper, we focus on nontrivial prefix sets.

A generalized prefix is any string over the extended prefix alphabet $\left\{\exists, \forall, \exists^{*}, \forall^{*}\right\}$. A prefix set $\mathcal{Q}$ is standard, if either $\mathcal{Q}=\{\exists, \forall\}^{*}$ or $\mathcal{Q}$ can be given by some generalized prefix.

For any prefix $Q$, the class $\operatorname{ESO}(Q)$ is the set of all $\Sigma_{1}^{1}$ formulas $\exists \mathbf{R} \varphi$, where $\varphi$ is a prenex first-order formula with prefix $Q$; for any prefix set $\mathcal{Q}$, the class $\operatorname{ESO}(\mathcal{Q})$ is the union $\operatorname{ESO}(\mathcal{Q})=\bigcup_{Q \in \mathcal{Q}} \operatorname{ESO}(Q)$.

For example, $\operatorname{ESO}\left(\exists^{*} \forall \exists^{*}\right)$ is the class of all formulas $\exists \mathbf{R} \exists \mathbf{y} \forall x \exists \mathbf{z} \varphi$, where $\varphi$ is quantifier-free; this is the class of ESO-prefix formulas, whose first-order part is in the well-known Ackermann class.

Recall that a literal is an atomic formula or the negation of such; equalities and inequalities are also literals. It is usual, when one deals with conjunctive normal forms, to define a clause as a set of literals interpreted as the disjunction of its members. We deal with disjunctive normal forms. Accordingly, we redefine a clause as set of literals interpreted as the conjunction of its members. A DNF formula can be seen as a set of clauses. For any formula $\Phi \in \operatorname{ESO}\left(\{\forall, \exists\}^{*}\right)$ whose quantifier-free part is a $\operatorname{DNF} \varphi=\bigvee_{i} \delta_{i}$, we denote by $\Delta(\Phi)$ (simply $\Delta$, if $\Phi$ is understood) the set of all clauses $\delta_{i}$ of $\varphi$.

Let $A=\left\{a_{1}, \ldots, a_{m}\right\}$ be a finite alphabet. A string over $A$ is a finite first-order structure $W=$ $\left\langle U, C_{a_{1}}^{W}, \ldots, C_{a_{m}}^{W}, S u c c^{W}, \min ^{W}, \max ^{W}\right\rangle$, for the vocabulary $\sigma_{A}=\left\{C_{a_{1}}, \ldots, C_{a_{m}}\right.$, Succ, $\left.\min , \max \right\}$, where

- $U$ is a nonempty finite initial segment $\{1,2, \ldots, n\}$ of the positive integers;

- each $C_{a_{i}}^{W}$ is a unary relation over $U$ (i.e., a subset of $U$ ), for $i=1, \ldots, m$ for the unary predicate $C_{a_{i}}$, also referred to as colors, such that the $C_{a_{i}}^{W}$ are pairwise disjoint and $\bigcup_{i} C_{a_{i}}^{W}=U$.

- $S u c c^{W}$ is the usual successor relation on $U$ and $\min ^{W}$ and $\max ^{W}$ are the first and the last element in $U$, respectively.

The strings $W$ for $A$ correspond to the nonempty finite words over $A$ in the obvious way; in abuse of notation, we often use $W$ in place of the corresponding word from $A^{*}$ and vice versa.

A SO sentence $\Phi$ over the vocabulary $\sigma_{A}$ is a second-order formula whose only free variables are the predicate variables of the signature $\sigma_{A}$, and where no constant symbols except min and max occur. Such a sentence defines a language over $A$, denoted $\mathcal{L}(\Phi)$, given by $\mathcal{L}(\Phi)=\{W \mid W \models \Phi\}$. We say that a language $L \subseteq A^{*}$ is expressed by $\Phi$, if $\mathcal{L}(\Phi)=L \cap A^{+}$(thus, for technical reasons, without loss of generality we disregard the empty string); $L$ is expressed by a set $S$ of sentences, if $L$ is expressed by some $\Phi \in S$. We say that $S$ captures a class $C$ of languages, if $S$ expresses all and only the languages in $C$.

Let $A$ be a finite alphabet. A sentence $\Phi$ over $\sigma_{A}$ is called regular, if $\mathcal{L}(\Phi)$ is a regular language. A set of sentences $S$ (in particular, any ESO-prefix class) is regular, if for every finite alphabet $A$, all sentences $\Phi \in S$ over $\sigma_{A}$ are regular.

Büchi [7] has shown the following fundamental theorem. Let MSO denote the fragment of second order 
logic in which all predicate variables have arity at most one, ${ }^{3}$ and let REG denote the class of regular languages.

\section{Proposition 2.1 (Büchi's Theorem) MSO captures REG.}

Note that Büchi's Theorem was independently found by B. Trakhtenbrot [53].

That MSO can express all regular languages is easy to see, since it is straightforward to describe the behavior of a finite state automaton by an existential MSO sentence. In fact, this is easily possible in monadic $\operatorname{ESO}(\forall \exists)$ as well as in monadic $\operatorname{ESO}(\forall \forall)$. Thus, we have the following lower expressiveness bound on ESO-prefix classes over strings.

Proposition 2.2 Let $\mathcal{Q}$ be any prefix set. If $\mathcal{Q} \cap\{\exists, \forall\}^{*} \forall\{\exists, \forall\}^{+} \neq \emptyset$, then $\operatorname{ESO}(\mathcal{Q})$ expresses all languages in REG.

\section{Nonregular ESO-Prefix Classes}

In this section, we present some ESO-prefix classes which are not regular. In particular, we show that $\operatorname{ESO}(\forall \forall \forall), \operatorname{ESO}(\forall \forall \exists)$, and $\operatorname{ESO}(\forall \exists \forall)$ include a well-known nonregular language. This means that whenever we have in a prefix $Q$ two universal FO quantifiers separated or followed by some other FO quantifier, then any class containing $Q$ is nonregular. As it will appear later, these three prefix-classes are the minimal nonregular standard prefix-classes of ESO.

Proposition 3.1 The language $L=\left\{a^{n} b^{n} \mid n \geq 0\right\}$ can be expressed by sentences

(i) $\exists R \forall x \forall y \exists z \cdot \varphi_{1}$,

(ii) $\exists R \forall x \forall y \forall z . \varphi_{2}$, and

(iii) $\exists R \forall x \exists y \forall z \cdot \varphi_{3}$,

where $R$ is binary and the $\varphi_{i}$ are quantifier-free.

Proof. The language $L$ is defined by the sentence

$$
\begin{aligned}
\Phi=\exists R \forall x, y \exists z \cdot & R(\min , \max ) \wedge C_{a}(\min ) \wedge \\
& {\left[R(x, y) \rightarrow\left(\neg R(y, x) \wedge\left(C_{a}(x) \leftrightarrow C_{b}(y)\right)\right)\right] \wedge } \\
& {\left[\left(R(x, y) \wedge C_{a}(x)\right) \rightarrow(\operatorname{Succ}(x, z) \wedge(z \neq y \rightarrow R(y, z)))\right] \wedge } \\
& {\left[\left(R(x, y) \wedge C_{b}(x)\right) \rightarrow(\operatorname{Succ}(z, x) \wedge R(y, z))\right] . }
\end{aligned}
$$

The formula says that $R$ is a directed graph such that an arc goes from $\min$ to $\max$, that the first letter of the word is an $a$, that $R$ is asymmetric and that arcs are between letters of different colors; the penultimate conjunct says that if an arc leads from an element $e_{a}$ colored with $C_{a}$ to an element $e_{b}$ colored with $C_{b}$, then

\footnotetext{
${ }^{3}$ Observe that we assume MSO allows one to use nullary predicate variables (i.e., propositional variables) along with unary predicate variables. Obviously, Büchi's Theorem survives.
} 
an arc must lead from $e_{b}$ to the successor $e_{a}$, unless the elements $e_{a}$ and $e_{b}$ are adjacent; the last conjunct states a similar condition for arcs from $C_{b}$ 's to $C_{a}$ 's.

To see that $\Phi$ works properly, consider first any word $W=a^{n} b^{n}$ in $L$. Then, $(W, R) \models \Phi$, where $R=\{(i, 2 n-i+1) \mid 1 \leq i \leq n\} \cup\{(2 n-i+1, i+1) \mid 1 \leq i<n\}$. Conversely, suppose that $(W, R) \models \Phi$, where $W=c_{1} c_{2} \cdots c_{m}$ and each $c_{i} \in\{a, b\}$. By induction on $i \leq\lfloor m / 2\rfloor$, show that the following holds:

$$
c_{i}=a, c_{m-i+1}=b, R(i, m-i+1), \text { and } R(m-i+1, i+1) .
$$

Thus, if $m$ is even, we have finished. Suppose then $m=2 n+1$ for some $n$, so that the given word $W$ is of the form $a^{n} c_{n+1} b^{n}$. In particular, we have $R(n, m-n+1)$ and $R(n, n+2)$. By $\Phi, R(n, n+2)$ implies $R(n+2, n+1)$, and $R(n+2, n+1) \wedge C_{b}(n+2)$ implies $C_{a}(n+1) \wedge R(n+1, n+1)$. However, $R(n+1, n+1)$ contradicts the asymmetry of $R$.

For $(i i)$, we slightly modify the previous formula $\Phi$ by turning the existential quantifier $\exists z$ into a universal quantifier $\forall z$, and by replacing the last two conjuncts with

$$
\left(R(x, y) \wedge C_{a}(x) \wedge S u c c(x, z) \wedge z \neq y\right) \rightarrow R(y, z)
$$

and

$$
\left(R(x, y) \wedge C_{b}(x) \wedge \operatorname{Succ}(z, x)\right) \rightarrow R(y, z),
$$

respectively.

For (iii), observe that with first-order quantifier prefix $\forall \exists \forall$, it is easy to say that $R$ describes a partioning of the string in 2-element sets $\left\{e_{1}, e_{2}\right\}$, such that $e_{1}$ has color $C_{a}$ and $e_{2}$ has color $C_{b}$ :

$$
\forall x \exists y \forall z\left[R(x, y) \wedge\left(C_{a}(x) \leftrightarrow C_{b}(y)\right) \wedge(R(x, z) \rightarrow R(z, x)) \wedge(R(x, z) \leftrightarrow z=y)\right]
$$

Indeed, observe that $R$ must be symmetric, and by the first and the last conjunct, we have $\forall x \exists ! y R(x, y)$. Moreover, it can be easily said with first-order prefix $\forall \exists \forall$ that a string $W$ is of the form $a^{n} b^{m}$ (say that every $C_{b}$ is followed by a $C_{b}$ ).

Corollary 3.1 The ESO-prefix classes $\operatorname{ESO}(\forall \forall \exists), \operatorname{ESO}(\forall \forall \forall)$, and $\operatorname{ESO}(\forall \exists \forall)$ express some nonregular languages.

In Section 9 we will derive by a more complicated proof even stronger results: the three ESO-prefix classes in Corollary 3.1 do not only express nonregular languages, they even express NP-complete languages.

Observe that the syntactic incomparability of ESO-prefix classes does not mean that their expressive capabilities over strings are incomparable. In particular, we show the following.

Proposition 3.2 Over strings, $\mathrm{ESO}(\forall \exists \forall)$ reduces to $\mathrm{ESO}(\forall \forall \forall)$. In other words, every language expressible in $\operatorname{ESO}(\forall \exists \forall)$ is expressible in $\operatorname{ESO}(\forall \forall \forall)$.

Proof. We begin with the following lemma.

Lemma 3.2 Let $P, Q$ be $(j+1)$-ary predicate symbols, $j \geq 0$, and let $\mathbf{x}$ be a $j$-tuple of individual variables. Then, $\forall \mathbf{x} \exists y P(\mathbf{x}, y)$ is over strings equivalent to $\exists Q \forall \mathbf{x} \forall y \forall z . \alpha$ for an appropriate quantifier-free formula $\alpha$. 
Proof of Lemma. Intuitively, $Q(\mathbf{x}, y)$ means that $P(\mathbf{x}, z)$ holds for some $z \leq y$. This can be expressed as follows:

$$
(Q(\mathbf{x}, \min ) \rightarrow P(\mathbf{x}, \min )) \wedge(\operatorname{Succ}(y, z) \rightarrow[Q(\mathbf{x}, z) \rightarrow(P(\mathbf{x}, z) \vee Q(\mathbf{x}, y))]) ;
$$

conjunct $Q(\mathbf{x}, \max )$ to this and get the desired $\alpha . \diamond$

To reduce $\operatorname{ESO}(\forall \exists \forall)$ to $\operatorname{ESO}(\forall \forall \forall)$, let

$$
\Phi=\exists \mathbf{R} \forall x \exists y \forall z \cdot \varphi
$$

where $\varphi$ is quantifier-free. Seconder-order skolemization gives an equivalent

$$
\Phi^{\prime}=\exists \mathbf{R} \exists F[\forall x \exists y F(x, y) \wedge \forall x \forall y \forall z(F(x, y) \rightarrow \varphi(x, y, z))] .
$$

Now use the lemma and then convert the resulting formula to the prenex form.

(Note that this proposition can not be applied in the proof of Proposition 3.1, since it introduces additional predicate variables in the formula.)

\section{A Combinatorial Theorem on Hypergraphs}

In this section, we prove a result on hypergraphs. This result may be of independent interest and will be used in Sections 5 and 7.

We introduce the concept of $[e]$-transversal of a (directed) hypergraph, which is a key concept in the proofs of the mains results in Sections 5 and 7. For understanding those proofs, it is necessary to be acquainted with the definitions of the present section.

An $r$-uniform directed hypergraph $H=(N, E)$ consists of an $r$-ary relation $E$ over finitely many elements of $N$, i.e., $E \subseteq 2^{N^{r}}$. (Note that other authors use the term directed hypergraph for a different concept.) $N$ is called the set of nodes and $E$ the set of (hyper)edges. Whenever we use the term hypergraph in this paper, we actually mean uniform directed hypergraphs. Directed (finite) graphs are a special case given by $r=2$.

We denote by $\operatorname{Pos}(a, e)$ the set of all positions at which $a$ occurs in edge $e$. Let, for instance $e=$ $\langle 5,6,4,5\rangle$, then $\operatorname{Pos}(5, e)=\{1,4\}, \operatorname{Pos}(6, e)=\{2\}, \operatorname{Pos}(8, e)=\emptyset$, and so on.

By abuse of notation, we often write $|H|$ instead of $|E|$ for a hypergraph $H=(N, E)$. Moreover, for two hypergraphs $H=(N, E)$ and $H^{\prime}=\left(N^{\prime}, E^{\prime}\right)$, we write $H \subseteq H^{\prime}$ iff $N \subseteq N^{\prime}$ and $E \subseteq E^{\prime}$.

Let $H=(N, E)$ a hypergraph. The degree $\operatorname{deg}_{H}(a)$ of a node $a \in N$ is the number of edges of $H$ in which $a$ occurs. This notion generalizes to sets $W$ of vertices: If $W \subseteq N$, then the $\operatorname{degree~} \operatorname{deg}_{H}(W)$ of set $W$ is the number of edges $e \in E$ that meet $W$, i.e. that have at least one component in $W$.

A transversal of a hypergraph $H=(N, E)$ is a set $T \subseteq N$ such that $T$ meets all $e \in E$. A transversal is minimal, if it is of minimal cardinality.

Let us now introduce a more sophisticated concept of transversal, the excluded edge transversal.

Definition 4.1 Let $H=(N, E)$ be a hypergraph and $e \in E$ an edge. Then, an [e]-transversal of $H$ is a subset $T$ of $N$ such that each edge $e^{\prime} \in E-\{e\}$ has at least one component $b$ so that $b \in T$ and $\operatorname{Pos}\left(b, e^{\prime}\right) \nsubseteq \operatorname{Pos}(b, e)$, i.e., $b$ either does not occur at all in $e$ or $b$ occurs in $e^{\prime}$ at least at one position where it does not occur in $e$. 
Note that each [e]-transversal of $H$ is a transversal of $(N, H-\{e\})$ but not vice-versa. Furthermore, if $T$ is an [e]-transversal of $H$, then every $U$ such that $T \subseteq U \subseteq N$ is an [e]-transversal of $H$.

An $[e]$-transversal is minimal, if it contains a minimal number of elements. We denote by $\tau_{e}(H)$ the cardinality of a minimal $[e]$-transversal of $H$.

Example 4.1 Let $H=(N, E)$ with $N=\{1,2,3,4,5\}$ and $E=\left\{e_{1}, \ldots, e_{6}\right\}$ :

\begin{tabular}{|c|c|c|c|c|}
\hline$e_{1}:$ & 1 & 1 & 2 & 4 \\
\hline$e_{2}:$ & 1 & 1 & 1 & 4 \\
\hline$e_{3}:$ & 5 & 4 & 3 & 2 \\
\hline$e_{4}:$ & 2 & 2 & 2 & 3 \\
\hline$e_{5}:$ & 1 & 2 & 2 & \\
\hline$e_{6}$ & 4 & 4 & 2 & \\
\hline
\end{tabular}

Then $T=\{1,2,4\}$ is an $\left[e_{1}\right]$-transversal of $H$, but $T^{\prime}=\{2,4\}$ and $T^{\prime \prime}=\{1,2\}$ are not, even though $T^{\prime}$ and $T^{\prime \prime}$ are both transversals of $(N, E-\{e\})$. Note that $T$ is even a minimal $\left[e_{1}\right]$-transversal of $H$. This can be seen as follows: Every $\left[e_{1}\right]$-transversal of $H$ must contain 1 to meet $e_{2}$ correctly and 4 to meet $e_{6}$ correctly. In order to meet $e_{4}$ it must contain either 2 or 3 . Therefore it must contain at least three elements. $T$ has three elements and is thus minimal. We have $\tau_{e_{1}}(H)=3$.

Definition 4.2 Let $H=(N, E)$ be a hypergraph. Then $t(H)$ is defined by

$$
t(H)=\frac{\sum_{e \in E} \tau_{e}(H)}{|H|} .
$$

Thus, $t(H)$ is the average minimal [e]-transversal size of the hypergraph $H$. The goal of this section is to show that the asymptotic growth of $t(H)$ is superlinear in $|H|$.

Let us first state two simple lemmas.

Lemma 4.1 Let $H^{\prime} \subseteq H$ and let e be an edge of $H^{\prime}$. Then $\tau_{e}\left(H^{\prime}\right) \leq \tau_{e}(H)$.

Proof. It suffices to note that every [e]-transversal of $H$ necessarily contains an $[e]$-transversal of $H^{\prime}$.

Lemma 4.2 Let $H=(N, E)$ and $H^{\prime}=\left(N^{\prime}, E^{\prime}\right)$ be two hypergraphs. If $H^{\prime} \subseteq H$ then $t\left(H^{\prime}\right) \cdot\left|H^{\prime}\right| \leq$ $t(H) \cdot|H|$.

Proof. We have

$$
t\left(H^{\prime}\right) \cdot\left|H^{\prime}\right|=\sum_{e \in E^{\prime}} \tau_{e}\left(H^{\prime}\right) \leq \sum_{e \in E^{\prime}} \tau_{e}(H) \leq \sum_{e \in E} \tau_{e}(H)=t(H) \cdot|H| .
$$

Here, the third expression follows by $H^{\prime} \subseteq H$ from Lemma 4.1.

We are now ready for the main result of this section. 
Theorem 4.3 For every positive integer $r$, there is a monotone polynomial $p$ such that every $r$-uniform hypergraph $H$ satisfies $|H|<p(t(H))$.

Proof. We prove the statement by induction on $r$.

(Induction Base) If $r=1$, then $|H|=t(H)+1$, and the statement trivially holds.

(Induction Step) Suppose that $p$ witnesses the claim for $r$, and let $H=(N, E)$ be an $r+1$-uniform hypergraph. Without loss of generality, we assume that $|H|>1$; by definition of $t(H)$, we then have $t(H) \geq 1$.

Since $t(H)$ is an average over all $\tau_{e}(H)$, there must exist an edge $e \in E$ such that $\tau_{e}(H) \leq t(H)$.

Let $T$ be a minimal $[e]$-transversal of $H$. It holds that $|T| \leq t(H)$. Since $T$ meets all edges of $H$ except possibly $e$, it holds that $\operatorname{deg}_{H}(T) \geq|H|-1 \geq|H| / 2$.

Consequently, there exists an element $a \in T$ such that $a$ occurs in at least $|H| /(2|T|)$ edges of $H$, i.e., $\operatorname{deg}_{H}(a) \geq|H| /(2|T|)$. Let $A$ be the set of all edges of $H$ containing $a$. We have $|A| \geq|H| /(2|T|)$.

The element $a$ may occur in different positions in the edges in $A$. However, since there are only $r+1$ positions, there must exist a position $i$ such that $a$ occurs in at least $|A| /(r+1) \geq|H| /(2|T|(r+1))$ elements of $A$ at position $i$. Let $B \subseteq A$ be the set of all edges in $A$ containing $a$ in the $i$-th position. Let $c:=1 / 2(r+1)$. Note that $c$ is a constant depending only on $r$ and that $|B| \geq|H| /(c|T|) \geq|H| /(c \cdot t(H))$.

Let $H^{\prime}=(N, B)$. Clearly, $H^{\prime} \subseteq H$. Retain that

$$
|H| \leq c \cdot\left|H^{\prime}\right| \cdot t(H) .
$$

Define $G:=\left(N, B_{a}\right)$ where

$$
B_{a}=\left\{\left\langle a_{1}, \ldots, a_{i-1}, a_{i+1}, \ldots, a_{r+1}\right\rangle \mid\left\langle a_{1}, \ldots, a_{r+1}\right\rangle \in B\right\} ;
$$

that is, $B_{a}$ is obtained from $B$ by dropping the $i$-th column (which uniformly contains $a$ in $B$ ).

Note that $|G|=\left|H^{\prime}\right|$ and, as easily seen, $t\left(H^{\prime}\right)=t(G)$.

Since $G$ is $r$-uniform, applying the assumption on $p$ yields

$$
\left|H^{\prime}\right|=|G|<p(t(G))=p\left(t\left(H^{\prime}\right)\right) .
$$

Furthermore,

$$
\begin{aligned}
|H| \cdot t\left(H^{\prime}\right) & \leq c \cdot\left|H^{\prime}\right| \cdot t(H) \cdot t\left(H^{\prime}\right) & & \text { by (1) } \\
& \leq c \cdot|H| \cdot t(H) \cdot t(H) & & \text { by Lemma } 4.2
\end{aligned}
$$

so that

$$
t\left(H^{\prime}\right) \leq c \cdot t(H) \cdot t(H) .
$$

Finally

$$
\begin{aligned}
|H| & \leq(c \cdot t(H)) \cdot\left|H^{\prime}\right| & & \text { by (1) } \\
& \leq(c \cdot t(H)) \cdot p\left(t\left(H^{\prime}\right)\right) & & \text { by (2) } \\
& \leq(c \cdot t(H)) \cdot p(c \cdot t(H) \cdot t(H)) & & \text { by (3) and the monotonicity of } p .
\end{aligned}
$$

It follows that the statement holds for $r+1$, which concludes the induction and the proof of the theorem.

Since any polynomial $p(n)$ is asymptotically dominated by $2^{n}$, we obtain from the previous theorem the following result.

Corollary 4.4 For each positive integer $r$, there exists a constant $c_{r} \geq 0$ such that for any $r$-uniform hypergraph $H$ with $|H| \geq c_{r}$, it holds that $t(H)>\log |H|$. 


\section{Successor-free $\operatorname{ESO}\left(\exists^{*} \forall \exists^{*}\right)$ is Regular}

In this and the following two sections, we prove that $\operatorname{ESO}\left(\exists^{*} \forall \exists^{*}\right)$ is regular. The proof is rather involved. In this section, we prove the regularity of the fragment of $\operatorname{ESO}\left(\forall \exists^{*}\right)$ that does not use Succ or min or max. In Section 6, we prove the regularity of the fragment $\operatorname{ESO}\left(\forall \exists^{*}\right)$ that does not use min or max. Finally, in Section 7, we prove the regularity of $\operatorname{ESO}\left(\exists^{*} \forall \exists^{*}\right)$.

In Subsection 5.1, we introduce concepts and notation used in the proof. In Subsection 5.2, we prove some normal form theorems. In Subsection 5.3, we introduce the semantic concept of boundedness and show that a sentence is regular if it is bounded. The main result of this section is then established in Subsection 5.4.

\subsection{Selectors, Witness Functions, Supports, and Conflicts}

Recall that, in this paper, a term is an individual variable or an individual constant. Call a clause $\varphi\left(x_{1}, \ldots, x_{n}\right)$ complete (or a complete type) with respect to a given vocabulary and a set of terms if it is a maximal syntactically consistent conjunction of literals in the given vocabulary with variables in $\left\{x_{1}, \ldots, x_{n}\right\}$.

Definition 5.1 (NF1) Let $A$ be an alphabet. $\operatorname{An} \operatorname{ESO}\left(\forall \exists^{*}\right)$ sentence $\Phi$ has the normal form 1, in short NF1, if its quantifier-free part consists of clauses complete for the vocabulary of $\Phi$ and for a set of individual variables (not necessarily all individual variables in $\Phi$ ).

Definition 5.2 Let $\Phi$ be an NF1 sentence $\exists \mathbf{R} \forall x \exists y_{1}, \ldots, y_{r} . \varphi$ with quantifier-free part $\varphi$, and let $W$ be a string with universe $U=U(W)$. A support for $(W, \Phi)$ is a pair $S=\langle\sigma, f\rangle$, where $\sigma$ and $f$ are as follows.

- $\sigma$ is a map: $U \longrightarrow \varphi$. Any such map is called a selector function for $(W, \Phi)$.

- $f$ is an $r$-tuple of functions $f_{i}: U \longrightarrow U$. Any such $r$-tuple is called a witness function for $(W, \Phi)$.

Given a support $V=\langle\sigma, f\rangle$ for $(W, \Phi)$, we introduce the following notation.

For any $a \in U$, the witness tuple for $a$ is the tuple $f(a)$, and $\operatorname{witset}(a, V)$ is the set $\left\{f_{i}(a) \mid 1 \leq i \leq r\right\}$ of witness elements for $a$.

For any $A \subseteq U$, wit $(A, V)$ is the set $\{f(a) \mid a \in A\}$ of the witness tuples for all elements in $A$, and wit $(V)$ is the set wit $(U, V)$ of all witness tuples according to $V$.

For any $\delta \in \Delta(\Phi), \operatorname{culp}(\delta, V)$ is the set $\sigma^{-1}(a)=\{a \in U \mid \sigma(a)=\delta\}$ of elements ("culprits") assigned to $\delta$ in $V$, and $w i t(\delta, V)$ is the set $\operatorname{wit}(\operatorname{culp}(\delta, V), V)$ of the witness tuples for all culprits for $\delta$.

For any $a \in U, \operatorname{lit}(a, V)$ is the collection of ground literals which results from replacing $x, y_{1}, \ldots, y_{r}$ with $a, f_{1}(a), \ldots, f_{r}(a)$ in $\sigma(a)$. If $A \subseteq U$, then $\operatorname{lit}(A, V)=\bigcup\{\operatorname{lit}(a, V): a \in A\}, \operatorname{lit}(\delta, V)=$ $\operatorname{lit}(\operatorname{culp}(\delta, V), V)$, and $\operatorname{lit}(V)=\operatorname{lit}(U, V)$.

For any $a \in U$, a free witness literal of $a$ is a literal in $\operatorname{lit}(a, V)$ that does not contain $a$. freelit $(a, V)$ is the set of free witness literals of $a$. If $A \subseteq U$, then freelit $(A, V)=\bigcup_{a \in A} \operatorname{freelit}(a, V)$ and freelit $(V)=$ freelit $(U, V)$.

Finally, $\operatorname{lit}(W)$ is the set of all ground literals true in $W$. 
Example 5.1 Consider a formula $\Phi=\exists R \forall x \exists y_{1}, y_{2}, y_{3} \cdot\left(\varphi_{1} \vee \varphi_{2} \vee \cdots\right)$, where

$$
\begin{aligned}
& \varphi_{1}=C_{a}(x) \wedge C_{a}\left(y_{1}\right) \wedge C_{b}\left(y_{2}\right) \wedge C_{b}\left(y_{3}\right) \wedge S u c c\left(x, y_{1}\right) \wedge S u c c\left(y_{2}, y_{3}\right) \wedge R\left(x, y_{2}\right) \wedge R\left(x, y_{3}\right) \ldots \\
& \varphi_{2}=C_{b}(x) \wedge C_{a}\left(y_{1}\right) \wedge C_{a}\left(y_{2}\right) \wedge C_{b}\left(y_{3}\right) \wedge S u c c\left(x, y_{1}\right) \wedge \neg R\left(y_{2}, y_{3}\right) \ldots
\end{aligned}
$$

Let $W$ be the string depicted below, and let $V=\langle\sigma, f\rangle$ be a support for $W$ and $\Phi$, such that $\sigma(2)=$ $\varphi_{1}, \sigma(11)=\varphi_{1}$, and $\sigma(24)=\varphi_{2}$, and where $f$ is defined on 2,11 , and 24 as depicted.

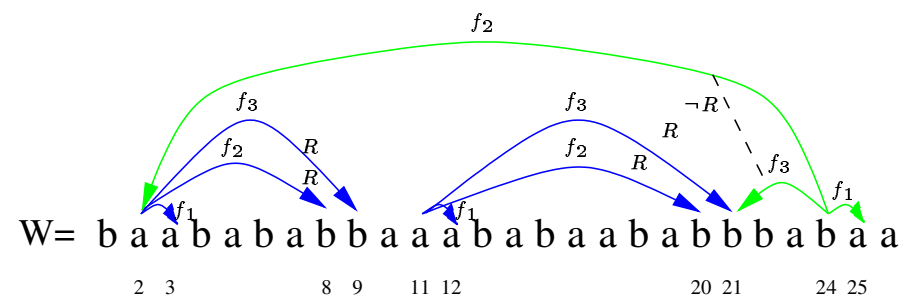

Then, $\operatorname{lit}(2, V)=\{R(2,8), R(2,9)\}, \operatorname{lit}(11, V)=\{R(11,20), R(11,21)\}$ and $\operatorname{lit}(24, V)=\{\neg R(2,21)\}$. Hence, $\operatorname{lit}(V) \supseteq\{R(2,8), R(2,9), R(11,20), R(11,21), \neg R(2,21)\}$.

The set $l i t(W)$ contains the literals $C_{b}(1), \neg C_{a}(1), C_{a}(2), \neg C_{b}(2), \ldots, C_{a}(26), \neg C_{b}(26), S u c c(1,2)$, $\operatorname{Succ}(2,3), \neg \operatorname{Succ}(1,3)$, etc; the literal $\neg R(2,21)$ belongs to freelit $(V)$.

A support $V$ for $(\Phi, W)$ is called locally consistent if, for each $a \in U$, the union $\operatorname{lit}(a, V) \cup \operatorname{lit}(W)$ is consistent. A support $V$ is called consistent, if the union $\operatorname{lit}(V) \cup \operatorname{lit}(W)$ is consistent; otherwise $V$ is inconsistent.

The following lemma is obvious.

Lemma 5.1 $W \models \Phi$ iff there exists a consistent support for $\Phi$ and $W$.

Elements $a, b$ of $W$ conflict over a support $W$ if the set $\operatorname{lit}(a, V) \cup \operatorname{lit}(b, V) \cup \operatorname{lit}(W)$ contains some atom $L$ together with its negation; such $L$ is a conflict induced by $a$ and $b$ over $V$. conf $(a, b, V)$ is the set of all conflicts induced by $a$ and $b$ over $V$. The following lemma is obvious.

Lemma 5.2 Let $V$ be a support for $(W, \Phi)$. $V$ is inconsistent if and only if there exists a pair $a, b \in U$ of elements conflicting over $V . V$ is locally inconsistent if and only if there exists an element a $\in U$ such that a conflicts with itself over $V$.

Remark 1 All these definitions remain valid for the full $\operatorname{ESO}\left(\forall \exists^{*}\right)$ (complete with Succ, $\min$ and $\max$ ).

\subsection{Further normal forms}

Definition 5.3 (NF2) An NF1 sentence $\Phi=\exists \mathbf{R} \forall x \exists y_{1}, \ldots, y_{r} \varphi$ is in normal form 2 (NF2), if and only if it fulfills the following conditions:

(a) If distinct variables $\chi, \xi$ occur in a clause, then the clause contains the inequality literal $\chi \neq \xi$.

(b) For each clause $\delta$, there exists a monadic predicate symbol $P$ in $\mathbf{R}$ such that $\delta$ contains the literal $P(x)$ and the literals $\neg P\left(y_{1}\right), \ldots, \neg P\left(y_{r}\right)$. 
Lemma 5.3 Let $G=(V, E)$ be a finite directed graph with a bound $r$ on the out-degree $\operatorname{deg}_{G}^{+}(v)=\mid\left\{v^{\prime} \in\right.$ $\left.V: v \rightarrow v^{\prime} \in E\right\} \mid$ of each node $v$. Then $G$ is $2 r+1$-colorable.

Proof. Induction on the number $n$ of nodes. The case $n=1$ is obvious. Suppose that the lemma has been proved for $n-1$. Since $r$ is the bound on the out-degree, the average in-degree is $\leq r$ and therefore the average (total) degree is $\leq 2 r$. Hence there is a node $v$ of degree $\leq 2 r$. By the induction hypothesis, the rest of the graph can be $(2 r+1)$-colored. The neighbors of $v$ use at most $2 r$ colors; an unused color can be used to color $v$.

A simple compactness argument shows that the lemma holds also for infinite graphs, but we will not use that result here.

Theorem 5.4 Every NF1 sentence $\Phi$ can be transformed into an equivalent (over strings) NF2 sentence $\Phi^{*}$.

Proof. Let $\Phi$ be as in the definition of NF1. To satisfy requirement (a), eliminate an equality $\chi=\xi$ or $\xi=\chi$, where $\chi$ precedes $\xi$ in the canonic ordering of variables, remove that literal and substitute $\chi$ for $\xi$ everywhere else in the clause.

To satisfy requirement (b), consider any string $W \models \Phi$ and any support $V$ for $(\Phi, W)$. Create a directed graph $G$ on $U(W)$ by linking every culprit $x$ to each of its witness elements. Obviously, the out-degrees are bounded by $r$; by Lemma 5.3, $G$ is $2 r+1$-colorable. Since this is true for any support $V$, the $(2 r+1)$ colorability follows from $\Phi$ itself. Introduce $2 r+1$ new monadic predicates $P_{1}, \ldots, P_{2 r+1}$ and replace each clause $\delta \in \Phi$ by a collection of new clauses augmenting $\delta$ with all possible "colorings" of all individual variables in $\delta$ by means of the predicates $P_{i}$ subject to the following restriction: $x$ has a color different from the colors of all other variables. (Coloring of a variable $\chi$ with color predicate $P_{i}$ means asserting $P_{i}(\chi)$ and $\neg P_{j}(\chi)$ for all $j \neq i$.) It is easy to see that the resulting sentence $\Phi^{*}$ is equivalent to $\Phi$.

Definition 5.4 (NF3) An NF2 sentence $\Phi=\exists \mathbf{R} \forall x \exists y_{1}, \ldots, y_{r} \varphi$ is in normal form 3 (NF3) if each clause contains all individual variables $x, y_{1}, \ldots, y_{r}$.

Theorem 5.5 For every sentence $\Phi=\exists \mathbf{R} \forall x \exists y_{1}, \ldots, y_{r} \varphi$, there exists an NF3 sentence $\Phi^{*}$ equivalent to $\Phi$ on strings of length at least $r+1$.

Proof. The goal is achieved in three steps.

Steps 1. If a clause $\delta$ of $\Phi$ does not contain all individual variables, augment it with literals $\chi \neq \xi$ where $\chi$ ranges over the variables in $\Phi$ and $\xi$ ranges over the variables missing in $\delta$. Let $\Phi_{1}$ be the result. Clearly $\Phi_{1}$ satisfies the condition (a) from the definition of NF2.

Step 2. Use the second part of the proof of Theorem 5.4 to transform $\Phi_{1}$ into $\Phi_{2}$ that is in NF2.

Step 3. If a clause of $\Phi_{2}$ is not complete with respect to the vocabulary of $\Phi$, replace it with an equivalent disjunction of complete clauses.

For reaching the objective of this section, it suffices to show that all NF3 sentences are regular.

Remark 2 This subsection also remains valid in the presence of Succ, min and $\max$. 


\subsection{Bounded sentences}

Let $K$ be a positive integer. An NF1 sentence $\Phi$ is $K$-bounded if, for each $W \models \Phi$, there exists a consistent support $V$ for $(W, \Phi)$ with $\mid$ wit $(V) \mid \leq K$, so that the total number of witness tuples is $\leq K$. $\Phi$ is bounded if it is $K$-bounded for some $K$.

Theorem 5.6 If an NF3 sentence $\Phi$ is bounded, then $\Phi$ is regular.

Proof. Suppose that an NF3 sentence $\Phi$ is $K$-bounded. We show that $\Phi$ is equivalent to a monadic second-order sentence, and thus, by Büchi's Theorem, $\Phi$ is regular.

Let $\Phi$ be of the form

$$
\exists \mathbf{R} \forall x \exists y_{1}, \ldots, y_{r} \bigvee_{\gamma \in \Delta} \gamma,
$$

where $\mathbf{R}$ is a list of predicate variables and the $\gamma$ are the clauses of $\Delta=\Delta(\Phi)$.

Note that whenever $(W, \Phi)$ has a consistent support, then there is also a consistent support $V$ for $(W, \Phi)$ such that at most $K \cdot r$ elements of $U(W)$ appear in all witness typles. Let $Z=\left\{z_{1}, \ldots, z_{K \cdot r}\right\}$ be a set of $K \cdot r$ fresh individual variables. Transform $\Phi$ to the following equivalent second-order sentence $\Phi^{\prime}$ :

$$
\exists z_{1}, \ldots, z_{K \cdot r} \exists \mathbf{R} \forall x \bigvee_{\gamma \in \Delta} \bigvee_{\zeta_{1}, \ldots, \zeta_{r} \in Z} \gamma\left[y_{1} / \zeta_{1}, \ldots, y_{r} / \zeta_{r}\right]
$$

where $y_{i} / \zeta_{i}$ means substitution of $\zeta_{i}$ for $y_{i}$. Let $\Delta^{\prime}$ be the set of all clauses in $\Phi^{\prime}$. Now it is not hard to see how all predicates of arity $>1$ can be eliminated from $\Phi^{\prime}$. For notational simplicity, we assume that $\mathbf{R}$ consists of one binary predicate $R$. Note that each $R$-literal occurring in $\Phi^{\prime}$ has all its arguments among $\{x\} \cup Z$. Replace each atom $R(\xi, \chi)$ by a new unary atom $R_{\xi, \chi}(x)$ or nullary atom $R_{\xi, \chi}$. That is, replace $R\left(z_{i}, z_{j}\right)$ by $R_{z_{i}, z_{j}}, R\left(z_{i}, x\right)$ by $R_{z_{i}, x}(x), R\left(x, z_{i}\right)$ by $R_{x, z_{i}}(x)$, and $R(x, x)$ by $R_{x, x}(x)$. A clause $\gamma \in \Delta^{\prime}$ yields a clause $\gamma^{*}$. Let $\mathbf{R}^{*}$ be the list of new monadic predicate symbols $R_{\xi, \chi}$ corresponding to the new atoms. Formula $\Phi^{\prime}$ is then equivalent to

$$
\exists z_{1}, \ldots, z_{K \cdot r} \exists \mathbf{R}^{*} \forall x\left(\psi \wedge \bigvee_{\gamma \in \Delta^{\prime}} \gamma^{*}\right),
$$

where $\forall x \psi$ asserts that the new predicates are properly correlated. $\psi$ is a conjunction of formulas like

$$
z_{2}=z_{3} \rightarrow\left(R_{x, z_{2}}(x) \leftrightarrow R_{x, z_{3}}(x)\right)
$$

or

$$
x=z_{2} \rightarrow\left(R_{z_{1}, x}(x) \leftrightarrow R_{z_{1}, z_{2}}\right) .
$$

This final formula is monadic.

\subsection{Successor-free NF1 sentences are regular}

In this subsection, we prove that every successor-free NF1 sentence $\Phi$ is regular. The crux of the proof is roughly described as follows. It is sufficient to show that $\Phi$ is bounded. To prove this, we take a string $W$ such that $\Phi \models W$ and a support $V$ for $\Phi$ and $W$ having a minimal extent (i.e, assigning - in a precise sense 
- a minimal number of witness tuples). Since the number of witness tuples (for short, witnesses) in $V$ is minimal, it is not possible to lump different witnesses together (i.e, we cannot replace the witness of one element by the witness of another element and thus decrease the number of witnesses). Since it is impossible to lump witnesses together, something must be responsible for this impossibility. Namely, for each element $a$ and each witness $w$ of another element different from its own witness $w_{0}$, there must exist at least one literal $L$ induced by $V$ that blocks the possibility of using $w$ as witness for $a$ instead of $w_{0}$. Such literals $L$ are called blockers. E.g., in Example 5.1, the literal $\neg R(2,21)$ is a blocker, because it blocks the reuse of the witnesses of position 11 for position 2 in the string $W$.

We compute a lower bound on the number of necessary blockers. We note that the set of blockers for a particular element $a$ having $w_{0}$ as witness corresponds to a $\left[w_{0}\right]$-transversal of the hypergraph $H$ formed by all witnesses. By Corollary 4.4, the size of this transversal is on average at least $\log |H|$. Moreover, it will be shown that the sets of blockers corresponding to different elements are disjoint. Therefore, $|H| \cdot \log |H|$ is a lower bound on the total number of blockers necessary to ensure the minimality of $V$. However, any support $V$ can induce only a linear number of literals (and thus blockers). By comparing the linear upper bound with the $|H| \cdot \log |H|$ lower bound, we conclude that the number of witnesses is constant; as a consequence, formula $\Phi$ is bounded.

Theorem 5.7 Every successor-free NF1 sentence is regular.

Proof. Let $\Phi=\exists \mathbf{R} \forall x \exists y_{1}, \ldots, y_{r} . \varphi$ be a successor-free NF1 sentence. By Theorem 5.5, we assume w.l.o.g. that $\Phi$ is in NF3.

We denote by $\ell$ the number of literals appearing in a clause of $\Phi$; note that since $\Phi$ is in NF3, all clauses of $\varphi$ have the same number of literals. For convenience, we write $\Delta$ as shorthand for $\Delta(\Phi)$.

We show that there exists a constant $K$ such that if $W \models \varphi$, then there exists a consistent support $V$ for $\Phi$ and $W$ such that $|w i t(V)| \leq K$. Thus $\Phi$ is bounded and by Theorem 5.6 regular.

Assume thus that $W \models \Phi$. Then let $V=\langle\sigma, f\rangle$ be a consistent support for $\Phi$ and $W$ such that the sum

$$
\sum_{\gamma \in \Delta}|w i t(\gamma, V)|
$$

is minimal over all consistent supports, i.e., for no other consistent support $V^{\prime}$ of $\Phi$ and $W$ this sum is smaller.

Let $\delta \in \Delta$ be a clause of $\varphi$, let $X=\operatorname{culp}(\delta, V)$, i.e., the set of elements in $U(W)$ which are mapped in $V$ by $\sigma$ to the clause $\delta$, and let $q=\mid$ wit $(\delta, V) \mid$.

Since $\Phi$ is in NF3, it fulfills part (b) of Definition 5.3, and for any element $a \in X$ and $b \in \operatorname{witset}(b, V)$ it holds $\sigma(b) \neq \sigma(a)$. Since all elements of $X$ have the same image $\delta$ under $\sigma$, the following property is true:

Fact $5.1 \forall a, b \in X, b \notin$ witset $(a, V)$.

We define an equivalence relation $\sim$ on $X$ as follows:

$$
\forall u, v \in X: u \sim v \text { iff } f(u)=f(v)
$$

Note that there are exactly $q=\mid$ wit $(\delta, V) \mid$ equivalence classes in $X / \sim$. Denote by $Z_{1}, \ldots, Z_{q}$ the different equivalence classes of $X / \sim$. 
For $a, b \in X$ we denote by $V[a \rightarrow \mathcal{W}(b)]$ the support obtained from $V$ by assigning to $a$ the witnesses of $b$ instead of its own witnesses. Formally, $V[a \rightarrow \mathcal{W}(b)]=\left\langle\sigma, f^{\prime}\right\rangle$ where $f^{\prime}(a)=f(b)$ and $f^{\prime}(v)=f(v)$ for each $v \in U(W) \backslash\{a\}$. Observe that literals in $\operatorname{lit}\left(V^{\prime}\right) \backslash \operatorname{lit}(V)$ where $V^{\prime}=V[a \rightarrow \mathcal{W}(b)]$ must involve $a$ and apart from $a$ only elements in witset $(b, V)$.

Claim 5.1 $\forall Z \in X / \sim \exists a \in Z \forall b \in X \backslash Z: V[a \rightarrow \mathcal{W}(b)]$ is inconsistent.

Proof of claim. Assume the claim does not hold. Then there exist a set $Z \in X / \sim$ and a function $h: Z \rightarrow X \backslash Z$ such that for each $a \in Z, V[a \rightarrow \mathcal{W}(h(a))]$ is consistent. Let $Z=\left\{c_{1}, \ldots, c_{k}\right\}$. We show that the support $V^{*}=\left\langle\sigma, f^{*}\right\rangle$, defined by

$$
V^{*}=V\left[c_{1} \rightarrow \mathcal{W}\left(h\left(c_{1}\right)\right)\right]\left[c_{2} \rightarrow \mathcal{W}\left(h\left(c_{2}\right)\right)\right] \ldots\left[c_{k} \rightarrow \mathcal{W}\left(h\left(c_{k}\right)\right)\right],
$$

is a consistent support for $\Phi$ and $W$.

To prove this, suppose $V^{*}$ is inconsistent. This inconsistency must be caused by two conflicting elements $a, b \in Z$. Indeed, let $\Lambda=\operatorname{lit}(U(W) \backslash Z, V)$; then, $\operatorname{lit}\left(V^{*}\right)=\Lambda \cup \bigcup_{a \in Z} \operatorname{lit}\left(a, V^{*}\right)$, where $\operatorname{lit}\left(a, V^{*}\right)=$ $\operatorname{lit}(h(a), V)[h(a) / a]$ is the set of literals $\operatorname{lit}(h(a), V)$ in which $h(a)$ is uniformly replaced by $a$. Since $\operatorname{lit}\left(V^{*}\right) \cup \operatorname{lit}(W)$ is inconsistent but, by choice of $h, \operatorname{lit}(V[a \rightarrow \mathcal{W}(h(a))]) \cup \operatorname{lit}(W)$ and thus its subset $\Lambda \cup \operatorname{lit}\left(a, V^{*}\right) \cup \operatorname{lit}(W)$ is consistent, it follows that for some $a, b \in Z$ it holds that $\operatorname{lit}\left(a, V^{*}\right) \cup \operatorname{lit}\left(b, V^{*}\right) \cup$ $\operatorname{lit}(W)$ is not consistent, i.e., $\operatorname{conf}\left(a, b, V^{*}\right) \neq \emptyset$. Moreover, $\operatorname{conf}\left(a, b, V^{*}\right)$ must contain two opposite literals $L$ and $L^{\prime}=\neg L$ that both either involve $a$ or involve $b$. In fact, if both $L, L^{\prime}$ would involve neither $a$ nor $b$, then $L, L^{\prime} \in \operatorname{lit}(h(a), V) \cup \operatorname{lit}(h(b), V) \cup \operatorname{lit}(W) \subseteq \operatorname{lit}(V) \cup \operatorname{lit}(W)$, which is a contradiction. Assume w.l.o.g. that both $L$ and $L^{\prime}$ involve $a$. Then $L, L^{\prime} \notin l i t\left(b, V^{*}\right)$, because by Fact 5.1, $a$ cannot occur in $f^{*}(b)=f(h(b))$ and thus $a$ does not occur in $\operatorname{lit}\left(b, V^{*}\right)$. Hence $L, L^{\prime} \in \operatorname{lit}\left(a, V^{*}\right) \cup \operatorname{lit}(W)$, and thus $\operatorname{lit}\left(a, V^{*}\right) \cup \operatorname{lit}(W)$ is inconsistent. Since $\operatorname{lit}\left(a, V^{*}\right) \cup \operatorname{lit}(W) \subseteq \operatorname{lit}(V[a \rightarrow \mathcal{W}(h(a))]) \cup \operatorname{lit}(W)$, this implies that $V[a \rightarrow \mathcal{W}(h(a))]$ is inconsistent. However, $V[a \rightarrow \mathcal{W}(h(a))]$ is asserted to be consistent; it follows that $V^{*}$ is consistent.

But by the definition of $\sim$ and $V^{*}$, we have $\left|w i t\left(\delta, V^{*}\right)\right|=\mid$ wit $(\delta, V) \mid-1$ and thus $\sum_{\gamma \in \Delta} \mid$ wit $\left(\gamma, V^{*}\right) \mid$ $<\sum_{\gamma \in \Delta} \mid$ wit $(\gamma, V) \mid$, which contradicts our minimality assumption on $V$. The claim is proved.

Choose for each $Z_{i}$ an element $a_{i} \in Z_{i}$ such that for each $b \in X \backslash Z_{i}, V_{i}=V\left[a_{i} \rightarrow \mathcal{W}(b)\right]$ is inconsistent. Let $w_{i}:=f\left(a_{i}\right)$, for each $1 \leq i \leq q$. Note that due to Fact A, for $1 \leq i, j \leq q$ it holds that $a_{i}$ does not occur in $w_{j}$.

It is easy to see that $V_{i}$ is locally consistent. Thus, a conflict in $V_{i}$ must involve a literal $L^{\prime}$ in

$$
\operatorname{lit}\left(V_{i}\right) \backslash \operatorname{lit}(V) \subseteq \operatorname{lit}\left(a_{i}, V_{i}\right) .
$$

Since $V_{i}$ is locally consistent, the literal $L$ which is the opposite of $L^{\prime}$ is in $\operatorname{lit}(V)$. Since $L^{\prime}$ is a new literal, it involves $a_{i}$. It is easy to see that $L^{\prime}$ cannot be unary or an equality (otherwise, it would belong to $V$ ). Since $L^{\prime} \notin \operatorname{lit}\left(a_{i}, V\right)$, it follows that some $b$ occurs in $w_{j}$ in a position where it does not occur in $w_{i}$. Notice also that both $L^{\prime}$ and $L$ contain apart from $a_{i}$ only elements from $w_{j}$

Fix such a literal $L$ and an element $b$ as described for $a_{i}$ and $w_{j}$ and refer to them as $\operatorname{blocker}\left(a_{i}, w_{j}\right)$ and an $\operatorname{dart}\left(a_{i}, w_{j}\right)$, respectively.

Let, for each $1 \leq i \leq q$,

$$
\begin{aligned}
& B\left(a_{i}\right)=\left\{\operatorname{blocker}\left(a_{i}, w_{j}\right) \mid 1 \leq j \leq q, j \neq i\right\}, \text { and } \\
& D\left(a_{i}\right)=\left\{\operatorname{dart}\left(a_{i}, w_{j}\right) \mid 1 \leq j \leq q, j \neq i\right\} .
\end{aligned}
$$


(Note that $D\left(a_{i}\right) \cap$ witset $\left(a_{j}, V\right) \neq \emptyset$, for all $1 \leq i \neq j \leq q$.) For $1 \leq i \leq q$, we have

$$
r \cdot\left|B\left(a_{i}\right)\right| \geq\left|D\left(a_{i}\right)\right| ;
$$

indeed, every element $e \in D\left(a_{i}\right)$ must occur in at least one blocker $\left(a_{i}, w_{j}\right) \in B\left(a_{i}\right)$, and at most $r$ elements $e \in D\left(a_{i}\right)$ can occur in a single blocker $\left(a_{i}, w_{j}\right)$. Moreover, from Fact A and taking into account the constants occurring in $B\left(a_{i}\right)$, it follows that

$$
B\left(a_{i}\right) \cap B\left(a_{j}\right)=\emptyset, \quad \text { for all } 1 \leq i \neq j \leq q .
$$

Consequently,

$$
\left|\bigcup_{i=1}^{q} B\left(a_{i}\right)\right|=\sum_{i=1}^{q}\left|B\left(a_{i}\right)\right| \geq \frac{1}{r} \sum_{i=1}^{q}\left|D\left(a_{i}\right)\right| .
$$

Let $H$ be the hypergraph $H=\left(U(W),\left\{w_{i} \mid 1 \leq i \leq q\right\}\right)$. Then, it is easy to see that for $1 \leq i \leq q, D\left(a_{i}\right)$ is a $\left[w_{i}\right]$-transversal of $H$. Hence, we have $\left|D\left(a_{i}\right)\right| \geq \tau_{w_{i}}(H)$ and thus

$$
\left|\bigcup_{i=1}^{q} B\left(a_{i}\right)\right|=\sum_{i=1}^{q}\left|B\left(a_{i}\right)\right| \geq \frac{1}{r} \sum_{i=1}^{q}\left|D\left(a_{i}\right)\right| \geq \frac{1}{r} \sum_{i=1}^{q} \tau_{w_{i}}(H)=\frac{t(H) \cdot|H|}{r}=\frac{t(H) \cdot q}{r} .
$$

Denote by $\operatorname{vert}(H)$ the set of all elements $b \in U(W)$ occurring in some edge of $H$ and let $\operatorname{lit}(H, V)=$ $\bigcup_{b \in \operatorname{vert}(H)} \operatorname{lit}(b, V)$.

We show the following:

- For all $1 \leq i, j \leq q$ such that $i \neq j$, it holds that blocker $\left(a_{i}, w_{j}\right)$ belongs to either $\operatorname{lit}(H, V)$ or freelit $(V)$.

To verify this, first observe from the properties of $L_{i, j}$ that blocker $\left(a_{i}, w_{j}\right) \in \operatorname{lit}\left(U(W)-\left\{a_{i}\right\}, V\right)$. It follows that if blocker $\left(a_{i}, w_{j}\right) \notin \operatorname{freelit}(V)$, then blocker $\left(a_{i}, w_{j}\right) \in \operatorname{lit}(b, V)$ for some $b \in U(W) \backslash\left\{a_{i}\right\}$ which occurs in $w_{j}$. This means blocker $\left(a_{i}, w_{j}\right) \in \operatorname{lit}(H, V)$, however.

Now let us determine how many blockers blocker $\left(a_{i}, w_{j}\right)$ must be at least in freelit $(V)$.

First observe that

$$
|\operatorname{lit}(H, V)| \leq \ell \cdot q \cdot r .
$$

(Recall that $\ell$ is the number of literals in a clause.)

It follows that

$$
\left|\bigcup_{i=1}^{q} B\left(a_{i}\right) \cap \operatorname{freelit}(V)\right| \geq \frac{t(H) \cdot q}{r}-\ell \cdot q \cdot r
$$

thus,

$$
\frac{t(H) \cdot q}{r}-\ell \cdot q \cdot r \leq|\operatorname{freelit}(V)| .
$$

Assume now without loss of generality that the $\delta \in \Delta$ we have chosen is such that $q=|\operatorname{wit}(\delta, V)|$ is maximal over all clauses $\delta \in \Delta$. Then,

$$
\mid \text { freelit }(V)|\leq| \Delta \mid \cdot q \cdot \ell .
$$


From these bounds on $\mid$ freelit $(V) \mid$, we derive that for maximal $q$, we have

$$
\frac{t(H) \cdot q}{r}-\ell \cdot q \cdot r \leq|\Delta| \cdot q \cdot \ell,
$$

whence

$$
t(H)-\ell \cdot r^{2} \leq|\Delta| \cdot \ell \cdot r .
$$

If $q>c_{r}$, then $|H|>c_{r}$, and since $H$ is $r$-uniform, by Corollary 4.4, we have $t(H)>\log |H|=\log q$. From this and the previous inequation we obtain $(\log q)-\ell \cdot r^{2} \leq|\Delta| \cdot \ell \cdot r$, and thus $\log q \leq \ell \cdot r \cdot(r+|\Delta|)$, from which it follows that

$$
q \leq 2^{\ell \cdot r \cdot(r+|\Delta|)} .
$$

Let $k_{0}=\max \left\{c_{r}, 2^{\ell \cdot r \cdot(r+|\Delta|)}\right\}$. It holds that $q \leq k_{0}$.

Now let $K=k_{0} \cdot|\Delta|$. Note that $K$ is a constant that depends only on formula $\Phi$.

Since $q$ is maximal, we have

$$
|w i t(V)| \leq \sum_{\gamma \in \Delta}|w i t(\gamma, V)| \leq|\Delta| \cdot q \leq k_{0} \cdot \Delta=K,
$$

which means that $|w i t(V)| \leq K$. This shows that $\Phi$ is bounded.

\section{$6 \operatorname{ESO}\left(\forall \exists^{*}\right)$ is Regular}

In this section, we show how Theorem 5.7 can be lifted to the case where the successor predicate is present.

Now, we assume that the (predefined) Succ predicate can occur in an NF1 sentence, but min and max may not occur; we will deal with the constants min and max in the next section.

\subsection{Generalized definitions}

In this subsection, we generalize the basic concepts and definitions from Section 5.1 in order to take the successor into account.

Let $\Phi=\exists \mathbf{R} \forall x \exists y_{1}, \ldots, y_{r} \varphi$ be a sentence (with Succ) in NF3. In the presence of successor, the witnesses $y_{1}, \ldots, y_{r}$ of a clause $\delta \in \Delta(\Phi)$ are split into two parts: the $y_{i}$ which are (directly or transitively) connected to $x$ via Succ, which we call the local witnesses of $x$, and all other $y_{i}$, which we call the remote witnesses of $x$.

Accordingly, if we have a consistent support $V=\langle\sigma, f\rangle$ for $\Phi$ and a string $W$, then $f(a)$, witset $(a, V)$, and $\operatorname{wit}(a, V)$ for any $a \in U(W)$ are split into a local and a remote part, which is designated by index $l$ and $r$, respectively.

More precisely, we have:

- $f_{l}(a)$ and $f_{r}(a)$ are the projections of $f(a)$ to the components which hold the local (resp. remote) witness elements of $a$ according to $\sigma(a)$; in particular, $f_{r}(a)=f(a)$ if $\delta$ has no local witnesses.

- witset $_{l}(a, V)$ (resp. witset $\left.(a, V)\right)$ is the set of all elements occurring in $f_{l}(a)$ (resp. $f_{r}(a)$ ).

- $\operatorname{wit}_{l}(A, V)=\left\{f_{l}(a) \mid a \in A\right\}$ and witt $_{r}(A, V)=\left\{f_{r}(a) \mid a \in A\right\}$, for any $A \subseteq U(W)$. 
- $\operatorname{wit}_{l}(\delta, V)=\operatorname{wit}_{l}(\operatorname{culp}(\delta), V)$ and wit $_{r}(\delta, V)=$ wit $_{r}(\operatorname{culp}(\delta), V)$, for every $\delta \in \Delta(\Phi)$;

- freelit $(a, V)$ is the set of all literals $L \in \operatorname{lit}(a, V)$ such that only elements from witset $_{r}(a, V)$ occur in $L$. In other words, neither $a$ nor any local witness of $a$ occurs in $L$.

Notice that $\operatorname{lit}(W)$ now also contains all interpreted literals for $S u c c$ which are true in $W$. The definition of local consistency and consistency of a support $V$ remains unchanged. We observe that the Lemmas 5.1 and 5.2 hold in the generalized setting.

\subsection{Fourth Normal Form}

For lifting the proof that $\operatorname{ESO}\left(\forall \exists^{*}\right)$ sentences are regular to the case with successor, we have to deal with local and remote witness elements in an appropriate way.

Notice that all local witness elements are uniquely determined by the culprit. Therefore, only remote witness elements can be reused. As we will show, whenever $W \models \Phi$, then a consistent support $V$ for $\Phi$ and $W$ exists which uses only a constant number of remote witness elements (where the constant depends only on the formula $\Phi^{\prime}$ ).

In order to deal with local witness elements, it is convenient to introduce a further normal form.

Definition 6.1 An $\operatorname{ESO}\left(\forall \exists^{*}\right)$ sentence $\Phi$ in NF3 has the normal form 4 (NF4), if it satisfies the following condition:

(c) For each clause $\delta \in \Delta(\Phi)$ and each local witness variable $y_{i}$, there exists a monadic predicate symbol $Q$ in $\mathbf{R}$ such that $Q\left(y_{i}\right)$ is a conjunct of $\delta$ while for each variable $y_{j}, 1 \leq j \neq i \leq r$ occurring in $\delta$, $\neg Q\left(y_{j}\right)$ is a conjunct of $\delta$.

Informally, condition (c) in the NF4 allows us to avoid locality conflicts when reusing witnesses; it prevents that local witness elements of $a \in \operatorname{culp}(\delta, V)$ are witness elements of any different $b \in \operatorname{culp}(\delta, V)$.

Theorem 6.1 For every NF1 sentence $\Phi=\exists \mathbf{R} \forall x \exists y_{1}, \ldots, y_{r} . \varphi$, where $\varphi$ is quantifier-free, there exists a sentence $\Phi^{*}$ in NF4 such that for each string $W$ of length at least $r+1, W \models \Phi$ iff $W \models \Phi^{*}$.

Proof. This can be established by following the proof of Theorem 5.5, by doing a little more coloring in the formula in the proof of Theorem 5.4. In the reduction to the graph coloring there, we add for each node $b$ in the graph $G$ on each occurrence of $b$ as a local witness element $r-1$ extra edges, directed from $b$ to all witness fellows for this occurrence; i.e., if $b \in$ witset $_{l}(a, V)$, then we add arcs to every node $b^{\prime} \in \operatorname{witset}(a, V) \backslash\{b\}$.

Since an element $b$ may only occur a bounded number of times as a local witness $(\leq c \cdot r$ for some constant $c$ ), the graph $G$ that we construct this way has out-degrees bounded by $c^{\prime} \cdot r^{2}$ for some constant $c^{\prime}$, and hence $G$ is $2 c^{\prime} \cdot r^{2}+1$-colorable for some constant $c^{\prime}$. Proceed then by asserting the coloring in the usual way.

We now have the following lemma, which generalizes Fact 5.1 in the proof of Theorem 5.7.

Lemma 6.2 Let $X=\operatorname{culp}(\delta, V)$ for some consistent support $V$ for a string $W$ and a $\delta \in \Delta(\Phi)$ where $\Phi$ is a formula in NF4. Then, the following holds:

$$
\forall a, b \in X: a \notin \operatorname{witset}(b, V) \wedge\left[a \neq b \Rightarrow \text { witset }_{l}(a, V) \cap \operatorname{witset}(b, V)=\emptyset\right] .
$$


Proof. From condition $(b)$ of NF4 ( $a$ and $b$ have the same color, and every element in witset $(b, V)$ has a color different from $b$ 's color), it follows $a \notin$ witset $(b, V)$. Suppose that for some $a, b \in X$ an element $c \in$ witset $_{l}(a, V) \cap$ witset $(b, V)$ exists. Suppose $c$ is colored red. By the coloring technique (condition (c) of NF4), all local witnesses elements of $a$ have a different color, and all remote witness elements have a color different from all local witness colors. Since $b \in X$ and $c \in \operatorname{witset}(b, X), c$ must be for $b$ at the same local witness position as for $a$. But this means that $b=a$, contradiction to the assumption. This proves the lemma.

\subsection{Bounded sentences with successor}

The concept of boundedness is generalized in the following way. An NF1 sentence $\Phi$ (with Succ) is bounded, if there exists a constant $K$ such that for each $W$ verifying $W \models \Phi$, there exists a consistent support $V$ for $\Phi$ and $W$ such that $\left|w_{i} t_{r}(V)\right| \leq K$, i.e, the number of remote witness parts in $V$ is at most $K$.

Theorem 5.6 is adapted as follows:

Theorem 6.3 Every NF4 formula $\Phi$ which is bounded is regular.

Proof. The proof is analogous to the proof of Theorem 5.6, but slightly more involved.

Let as in the proof of Theorem 5.6 be $Z=\left\{z_{1}, \ldots, z_{k}\right\}$ the fresh variables for the remote witness elements. Transform $\Phi$ to $\Phi^{\prime}$ :

$$
\exists z_{1}, \ldots, z_{k} \exists \mathbf{R} \forall x \exists y_{1}, \ldots, y_{r} \bigvee_{\gamma \in \Delta(\Phi)} \bigvee_{\zeta_{1}, \ldots, \zeta_{r} \in Z} \gamma\left[\vartheta_{1}, \ldots, \vartheta_{r}\right]
$$

where $\vartheta_{i}$ stands for $y_{i} / \zeta_{i}$, if $y_{i}$ is a remote witness variable for $\gamma$, and for $y_{i} / y_{i}$ (i.e., replace $y_{i}$ by itself) if $y_{i}$ is a local witness variable for $\gamma$.

The replacement of binary atoms $R(\xi, \chi)$ by new monadic atoms $R_{\xi, \chi}(x)$ is done in a similar way, but for each local witness $y_{i}$ from $\xi$, $\chi$, we must record in $R_{\xi, \chi}$ the local position of $y_{i}$ to $x$ w.r.t. Succ; this can be handled if we assume w.l.o.g. that $y_{1}, y_{3}, \ldots, y_{2 i+1}, \ldots$ (odd index) is the first, second, $i$-th successor of $x$ and $y_{2}, y_{4}, \ldots, y_{2 i}, \ldots$ (even index) is the first, second, $i$-th element before $x$.

For example, $R_{y_{2}, z_{1}}(x)$ then tells that the predecessor of $x$ is in relation $R$ to element $z_{1}$, and $R_{y_{3}, x}(x)$ that the second element after $x$ is in relation $R$ to $x$.

The formula $\psi$ ensures the compatibility of the monadic predicates that we have introduced, and again consists of a conjunction of FO formulas for each pair of new predicates $R_{\xi, \chi}$ and $R_{\xi^{\prime}, \chi^{\prime}}$.

For the previous two predicates, that formula would look like:

$$
\begin{aligned}
\forall x, y_{1}, \ldots, y_{r} \forall u, v_{1}, \ldots, v_{r}([ & \operatorname{Succ}\left(y_{1}, x\right) \\
\operatorname{Succ}\left(v_{1}, v_{3}\right) & \left.\left.\wedge\left(y_{2}=v_{3}\right) \wedge\left(z_{1}=x\right)\right] \rightarrow\left[R_{y_{2}, z_{1}}(x) \leftrightarrow R_{y_{3}, x}(u)\right]\right)
\end{aligned}
$$

(Variables $u, v_{1}, \ldots, v_{r}$ replace $x, y_{1}, \ldots, y_{r}$ for accessing the $R_{y_{3}, x}$.)

Repeating this process again gives a monadic formula $\Phi^{*}$, and proves the theorem. 


\subsection{NF1 sentences with successor are regular}

We now prove the following generalization of Theorem 5.7.

Theorem 6.4 Every NF1 sentence with possible occurrences of Succ is regular.

Proof. The proof is similar to the proof of Theorem 5.7, but now we have to deal with possible inconsistencies due to the successor.

Let $\Phi=\exists \mathbf{R} \forall x \exists y_{1}, \ldots, y_{r} \varphi$ be an NF1 sentence. By Theorem 6.1, we assume w.l.o.g. that $\Phi$ is in NF4.

We show that there exists a constant $K$ such that if $W \models \varphi$, then there exists a consistent support $V$ for $\Phi$ and $W$ such that $\mid$ wit $_{r}(V) \mid \leq K$. Thus $\Phi$ is bounded and by Theorem 6.3 regular.

Assume thus that $W \models \Phi$. Then let $V=\langle\sigma, f\rangle$ be a consistent support for $\Phi$ and $W$ such that the sum

$$
\sum_{\gamma \in \Delta}\left|w i t_{r}(\gamma, V)\right|
$$

is minimal over all consistent supports, i.e., for no other consistent support $V^{\prime}$ of $\Phi$ and $W$ this sum is smaller.

Let $\delta \in \Delta$ be a clause of $\varphi$, let $X=\operatorname{culp}(\delta, V)$, i.e., the set of elements in $U(W)$ which are mapped in $V$ by $\sigma$ to the clause $\delta$, and let $q=\mid$ wit $(\delta, V) \mid$.

By Lemma 6.2, we have the following:

Fact 6.1 $\forall a, b \in X: a \notin \operatorname{witset}(b, V) \wedge\left[a \neq b \Rightarrow \operatorname{witset}_{l}(a, V) \cap \operatorname{witset}(b, V)=\emptyset\right]$.

We define an equivalence relation $\sim$ on $X$ as follows:

$$
\forall a, b \in X: a \sim b \text { iff } f_{r}(a)=f_{r}(b)
$$

i.e., the elements $a$ and $b$ which have the same remote witness parts are in the same class. Note that there are exactly $q=\mid$ wit $_{r}(\delta, V) \mid$ equivalence classes in $X / \sim$. Denote by $Z_{1}, \ldots, Z_{q}$ the different equivalence classes of $X / \sim$.

For $a, b \in X$ we denote by $V\left[a \rightarrow \mathcal{W}_{r}(b)\right]$ the support obtained from $V$ by assigning to $a$ the remote witnesses of $b$ instead of its own remote witnesses. Formally, if $V=\langle\sigma, f\rangle$, then $V\left[a \rightarrow \mathcal{W}_{r}(b)\right]=\left\langle\sigma, f^{\prime}\right\rangle$ such that $f^{\prime}(v)=f(v)$, for every $v \in U(W) \backslash\{a\}$, and $f^{\prime}(a)$ is the (unique) tuple such that $f_{l}^{\prime}(a)=f_{l}(a)$ and $f_{r}^{\prime}(a)=f_{r}(b)$.

Compared to $V$, any new literal $L$ in $\operatorname{lit}\left(V\left[a \rightarrow \mathcal{W}_{r}(b)\right]\right)$ must involve elements from $\operatorname{witset}_{l}(a, V) \cup$ $\{a\}$ and also elements from $f_{r}(b)$, i.e., witset $t_{r}(b, V)$. To see this, note by the definition of $V^{\prime}$, clearly all new literals must be from $\operatorname{lit}\left(a, V^{\prime}\right)$; since $\operatorname{lit}\left(a, V^{\prime}\right)$ results from $l i t(b, V)$ by substituting $a$ and wit $_{l}(a, V)$ for $b$ and $w_{i t}(b, V)$, and since, by Fact 6.1, neither $a$ nor any local witness of $a$ is a witness of $b$, any literal $L \in \operatorname{lit}\left(a, V^{\prime}\right)$ which does neither contain $a$ nor any element of $\operatorname{witset}_{l}(a, V)$ belongs to $\operatorname{lit}(b, V)$, and thus $L$ is not new. Hence, all new literals of $V^{\prime}$ w.r.t. $V$ must contain an element of witset $_{l}(a) \cup\{a\}$, and apart from $a$ also some element in witset $_{r}(b, V)$.

Claim 6.1 $\forall Z \in X / \sim \exists a \in Z \forall b \in X \backslash Z: V\left[a \rightarrow \mathcal{W}_{r}(b)\right]$ is inconsistent. 
Proof of claim. Assume the claim does not hold. Then there exist a set $Z \in X / \sim$ and a function $h: Z \rightarrow X \backslash Z$ such that for each $a \in Z, V\left[a \rightarrow \mathcal{W}_{r}(h(a))\right]$ is consistent. Let $Z=\left\{c_{1}, \ldots, c_{k}\right\}$. We show that the support $V^{*}=\left\langle\sigma, f^{*}\right\rangle$, defined by

$$
V^{*}=V\left[c_{1} \rightarrow \mathcal{W}_{r}\left(h\left(c_{1}\right)\right)\right]\left[c_{2} \rightarrow \mathcal{W}_{r}\left(h\left(c_{2}\right)\right)\right] \ldots\left[c_{k} \rightarrow \mathcal{W}_{r}\left(h\left(c_{k}\right)\right)\right],
$$

is a consistent support for $\Phi$ and $W$.

To prove this, suppose $V^{*}$ is inconsistent. This inconsistency must be caused by two conflicting elements $a, b \in Z$. Indeed, let $\Lambda=\operatorname{lit}(U(W) \backslash Z, V)$; then, $\operatorname{lit}\left(V^{*}\right)=\Lambda \cup \bigcup_{e \in Z} \operatorname{lit}\left(e, V^{*}\right)$, where $\operatorname{lit}\left(a, V^{*}\right)=$ $\operatorname{lit}(h(a), V)[h(a) / a]$ is the set of literals $\operatorname{lit}(h(a), V)$ in which $h(a)$ is uniformly replaced by $a$. Since $\operatorname{lit}\left(V^{*}\right) \cup \operatorname{lit}(W)$ is inconsistent but, by choice of $h, \operatorname{lit}\left(V\left[a \rightarrow \mathcal{W}_{r}(h(a))\right]\right) \cup \operatorname{lit}(W)$ and thus its subset $\Lambda \cup \operatorname{lit}\left(a, V^{*}\right) \cup \operatorname{lit}(W)$ is consistent, it follows that for some $a, b \in Z$ it holds that $\operatorname{lit}\left(a, V^{*}\right) \cup \operatorname{lit}\left(b, V^{*}\right) \cup$ $\operatorname{lit}(W)$ is not consistent, i.e., $\operatorname{conf}\left(a, b, V^{*}\right) \neq \emptyset$.

Let $\{L, \neg L\} \in \operatorname{conf}\left(a, b, V^{*}\right)$ be a conflict of $a$ and $b$. Since every $V\left[c_{i} \rightarrow \mathcal{W}_{r}\left(h\left(c_{i}\right)\right)\right]$ is locally consistent, also $V^{*}$ is locally consistent, and thus we conclude that $a \neq b$ and $L, \neg L \notin \operatorname{lit}(W)$.

Moreover, both $L$ and $L^{\prime}=\neg L$ must contain an element from either $S_{a}=\operatorname{witset}_{l}(a, V) \cup\{a\}$ or from $S_{b}=$ witset $_{l}(b, V) \cup\{b\}$; if they would contain neither, then $L, L^{\prime} \in \operatorname{lit}(V)$ would hold, which contradicts the consistency of $V$. Suppose then that $L, L^{\prime}$ contain an element from $S_{a}$. Then, $L, L^{\prime} \notin \operatorname{lit}\left(b, V^{*}\right)$, since, by Fact 6.1, no element from $S_{a}$ can be a witness element of $b$. This is a contradiction. An analogous contradiction arises for assuming that $L, L^{\prime}$ contain an element from $S_{b}$. It follows that $V^{*}$ is consistent.

But by the definition of $\sim$ and $V^{*}$, we have $\left|w i t\left(\delta, V^{*}\right)\right|=\mid$ wit $(\delta, V) \mid-1$ and thus $\sum_{\gamma \in \Delta} \mid$ wit $\left(\gamma, V^{*}\right) \mid$ $<\sum_{\gamma \in \Delta} \mid$ wit $(\gamma, V) \mid$, which contradicts our minimality assumption on $V$. The claim is proved.

Choose for each $Z_{i}$ an element $a_{i} \in Z_{i}$ such that for each $b \in X \backslash Z_{i}, V\left[a_{i} \rightarrow \mathcal{W}_{r}(b)\right]$ is inconsistent. Let $w_{i}:=f\left(a_{i}\right)$, for each $1 \leq i \leq q$. Note that due to Fact 6.1 , for every $1 \leq i \neq j \leq q$, no element from witset $_{l}(a, V) \cup\{a\}$ occurs in $f(b)$, i.e., in $\operatorname{witset}(b, V)$.

The inconsistency of $V\left[a_{i} \rightarrow \mathcal{W}_{r}\left(a_{j}\right)\right]$ implies that there is a pair of opposite literals $L_{i, j}, L^{\prime}=\neg L_{i, j} \in$ $\operatorname{lit}\left(V\left[a_{i} \rightarrow \mathcal{W}_{r}\left(a_{j}\right)\right]\right) \cup \operatorname{lit}(W)$. Since $\Phi$ has NF4, it follows that either

(i) the predicate symbol of $L_{i, j}$ is $S u c c$, or

(ii) the predicate symbol of $L_{i, j}$ is some predicate $P$ of arity $>1$ different from Succ and equality.

Indeed, all monadic literals in $\operatorname{lit}\left(V\left[a_{i} \rightarrow \mathcal{W}_{r}\left(a_{j}\right)\right]\right)$ occur in $\operatorname{lit}(V)$, and by Fact $6.1 a_{i}$ and its local witnesses are disjoint from $a_{j}$ and its witnesses, which makes an equality conflict impossible.

Fact 6.1 tells us that for a particular $j$, only for a small number of $i$ an $L_{i, j}$ as in $(i)$ is possible. Namely, an $L_{i, j}$ as in $(i)$ implies that the contiguous segment $S e g$ constituted by witset $_{l}\left(a_{i}, V\right) \cup\left\{a_{i}\right\}$ is in $\operatorname{lit}\left(a_{i}, V\left[a_{i} \rightarrow \mathcal{W}_{r}\left(a_{j}\right)\right]\right)$ adjacent to some maximal contiguous segment $S^{\prime} g^{\prime}$ constituted by elements in witset $_{r}(b, V)$; note that an overlap between $S e g$ and $S_{e g}$ is not possible. Since the number of maximal contiguous segments in witset $_{r}(b, V)$ is clearly bounded by $r$, for at most $2 r$ elements $a_{i}$ statement $(i)$ applies. In the other cases, $(i i)$ must be true.

Suppose that $(i i)$ is true. Then, the following properties of $L_{i, j}, L_{i, j}^{\prime}$ can be derived:

- both $L_{i, j}$ and $L_{i, j}^{\prime}$ involve some element from $S_{a_{i}}=$ witset $_{l}\left(a_{i}\right) \cup\left\{a_{i}\right\}$. For, otherwise $L_{i, j}$ and $L_{i, j}^{\prime}$ would already have existed in $\operatorname{lit}(V) \cup \operatorname{lit}(W)$. Similarly,

- at least one of $L_{i, j}, L_{i, j}^{\prime}$ must be from $\operatorname{lit}\left(a_{i}, V\left[a_{i} \rightarrow \mathcal{W}_{r}\left(a_{j}\right)\right]\right)$. 
- $L_{i, j}, L_{i, j}^{\prime} \notin \operatorname{lit}(W)$. Immediate.

- not both $L_{i, j}$ and $L_{i, j}^{\prime}$ appear in $\operatorname{lit}\left(a_{i}, V\left[a_{i} \rightarrow \mathcal{W}_{r}\left(a_{j}\right)\right]\right)$. This follows from Fact 6.1 and the local consistency of $V . \operatorname{lit}\left(a_{j}, V\right)$.

Assume then w.l.o.g. that $L_{i, j} \notin \operatorname{lit}\left(a_{i}, V\left[a_{i} \rightarrow \mathcal{W}_{r}\left(a_{j}\right)\right]\right)$ and $L_{i, j}^{\prime} \in \operatorname{lit}\left(a_{i}, V\left[a_{i} \rightarrow \mathcal{W}_{r}\left(a_{j}\right)\right]\right)$. Since $L_{i, j} \in \operatorname{lit}\left(V\left[a_{i} \rightarrow \mathcal{W}_{r}\left(a_{j}\right)\right]\right) \backslash \operatorname{lit}(W)$, it follows that $L_{i, j} \in \operatorname{lit}\left(U(W) \backslash\left\{a_{i}\right\}, V\right)$ and thus $L_{i, j} \in \operatorname{lit}(V)$. On the other hand, $L_{i, j}^{\prime} \notin \operatorname{lit}(V)$, for otherwise $V$ would be inconsistent.

Let $w_{j}=f_{r}\left(a_{j}\right)$, for $j=1, \ldots, q$. We have the further properties of $L_{i, j}$ and $L_{i, j}^{\prime}$.

- $L_{i, j}$ and $L_{i, j}^{\prime}$ must contain some element $b$ from witset $_{r}\left(a_{j}, V\right)$ from some position of $w_{j}$ at which in $w_{i}$ no $b$ is present. To see this, assume the contrary. Then since $\sigma\left(a_{i}\right)=\sigma\left(a_{j}\right)=\delta, L_{i, j}^{\prime} \in \operatorname{lit}\left(a_{i}, V\right)$ follows and thus $L_{i, j}, L_{i, j}^{\prime} \in \operatorname{lit}(V)$. Contradiction. (Observe that from NF4 of $\Phi$ and condition (a) of Definition 5.3, each element $v \in U(W)$ can occur at most once in any $w_{k}$, and hence $\left|\operatorname{Pos}\left(v, w_{k}\right)\right| \leq$ 1.)

- $L_{i, j}, L_{i, j}^{\prime}$ contain apart from elements in $S_{a_{i}}$ only elements from witset $_{r}\left(a_{j}\right)$. (Indeed, $L_{i, j}^{\prime}=\neg L_{i, j}$ is in $\operatorname{lit}\left(a_{i}, V\left[a_{i} \rightarrow \mathcal{W}_{r}\left(a_{j}\right)\right]\right)$. (ii).

Call a pair $a_{i}, a_{j}$ Succ-consistent, if $V\left[a_{i} \rightarrow \mathcal{W}_{r}\left(a_{j}\right)\right]$ does not have any inconsistency $L_{i, j}, L_{i, j}^{\prime}$ of type

For each $1 \leq i, j \leq q$ such that $a_{i}, a_{j}$ are Succ-consistent, fix a literal $L_{i, j}$ and element $b$ as described and refer to them as $\operatorname{blocker}\left(a_{i}, w_{j}\right)$ and $\operatorname{dart}\left(a_{i}, w_{j}\right)$, respectively.

Let, for each $1 \leq i \leq q$,

$$
\begin{aligned}
& B\left(a_{i}\right)=\left\{\operatorname{blocker}\left(a_{i}, w_{j}\right) \mid 1 \leq j \neq i \leq q, a_{i}, a_{j} \text { are Succ-consistent }\right\}, \text { and } \\
& D\left(a_{i}\right)=\left\{\operatorname{dart}\left(a_{i}, w_{j}\right) \mid 1 \leq j \neq i \leq q, a_{i}, a_{j} \text { are Succ-consistent }\right\} .
\end{aligned}
$$

Notice that $\operatorname{blocker}\left(a_{i}, w_{j}\right)$ contains only elements from witset $_{l}(a, V) \cup\left\{a_{i}\right\}$ and from $w_{j}$ (i.e., witset $_{r}\left(a_{j}\right)$ ), and at least some from witset $_{l}(a, V) \cup\left\{a_{i}\right\}$ and does contain $\operatorname{dart}\left(a_{i}, w_{j}\right)$.

For $1 \leq i \leq q$, we have

$$
r \cdot\left|B\left(a_{i}\right)\right| \geq\left|D\left(a_{i}\right)\right|
$$

indeed, every element $e \in D\left(a_{i}\right)$ must occur in at least one blocker $\left(a_{i}, w_{j}\right) \in B\left(a_{i}\right)$, and at most $r$ elements $e \in D\left(a_{i}\right)$ can occur in a single blocker $\left(a_{i}, w_{j}\right)$. Moreover, from Fact 6.1 and the elements occurring in $B\left(a_{i}\right)$, it follows that

$$
B\left(a_{i}\right) \cap B\left(a_{j}\right)=\emptyset, \quad \text { for all } 1 \leq i \neq j \leq q .
$$

Consequently,

$$
\left|\bigcup_{i=1}^{q} B\left(a_{i}\right)\right|=\sum_{i=1}^{q}\left|B\left(a_{i}\right)\right| \geq \frac{1}{r} \sum_{i=1}^{q}\left|D\left(a_{i}\right)\right| .
$$

Let $H$ be the hypergraph $H=\left(U(W),\left\{w_{i} \mid 1 \leq i \leq q\right\}\right)$, and $H_{i} \subseteq H$ the hypergraph $H_{i}=\left\{w_{j} \mid a_{i}, a_{j}\right.$ are Succ-consistent \}, for $1 \leq i \leq q$,

It is easily verified that for every $1 \leq i \leq q$, the set $D\left(a_{i}\right)$ is a $\left[w_{i}\right]$-transversal of $H_{i}$.

Every $\left[w_{i}\right]$-transversal $T$ of $H_{i}$ can be extended to a $\left[w_{i}\right]$-transversal of $H$. (Indeed, consider $w_{j} \in H \backslash H_{i}$. Since $w_{i}=f_{r}\left(a_{i}\right) \neq f_{r}\left(a_{k}\right)=w_{j}$, there is a position where $w_{i}$ and $w_{j}$ differ; pick an element $b_{j}$ in that 
position in $w_{j}$. By adding all $b_{j}$ 's to $T$, we obtain a $\left[w_{i}\right]$-transversal of $H$.) Thus if we set $d_{i}=|H|-\left|H_{i}\right|$, we obtain the following inequation:

$$
\tau_{w_{i}}\left(H_{i}\right)+d_{i} \geq \tau_{w_{i}}(H)
$$

Indeed, we need to add at most $d_{i}$ elements to a minimal $\left[w_{i}\right]$-transversal of $H_{i}$ to obtain a $\left[w_{i}\right]$-transversal of $H$.

By the above considerations on the predicate in literal $L_{i, j}$, for every $w_{j}$ at most $2 r a_{i}$ exist such that a Succ-conflict between $a_{i}$ and $a_{j}$ is present in $V\left[a_{i} \rightarrow \mathcal{W}_{r}\left(a_{j}\right)\right]$. Hence, we obtain

$$
\begin{aligned}
\sum_{w_{i} \in H}\left|D\left(a_{i}\right)\right| & \geq \sum_{w_{i} \in H} \tau_{w_{i}}\left(H_{i}\right) \\
& \geq \sum_{w_{i} \in H}\left(\tau_{w_{i}}(H)-d_{i}\right) \\
& \geq\left(\sum_{w_{i} \in H} \tau_{w_{i}}(H)\right)-2 \cdot q \cdot r \\
& =t(H) \cdot|H|-2 \cdot q \cdot r .
\end{aligned}
$$

Thus, we obtain from (4) and (6) the following bound on the number of blocking literals:

$$
\left|\bigcup_{i=1}^{q} B\left(a_{i}\right)\right| \geq \frac{t(H) \cdot q}{r}-2 \cdot q .
$$

We show the following:

- For every $1 \leq i \neq j \leq q$ such that $a_{i}, a_{j}$ are Succ-consistent, it holds that either

$(\alpha)$ blocker $\left(a_{i}, w_{j}\right) \in \operatorname{freelit}(V)$, or

$(\beta)$ there exist $b, b^{\prime} \in U(W)$ such that $b^{\prime}$ occurs in blocker $\left(a_{i}, w_{j}\right)$, $b^{\prime} \in$ witset $_{l}(b) \cup\{b\}$, and blocker $\left(a_{i}, w_{j}\right) \in \operatorname{lit}(b, V)$.

To verify this, recall that blocker $\left(a_{i}, w_{j}\right) \in \operatorname{lit}(V)$; hence, if $\operatorname{blocker}\left(a_{i}, w_{j}\right) \notin \operatorname{freelit}(V)$, then some $b \in U(W)$ must exist such that $\operatorname{blocker}\left(a_{i}, w_{j}\right) \in \operatorname{lit}(b, V) \backslash$ freelit $(V)$. Thus, some $b^{\prime} \in$ witset $_{l}(b) \cup\{b\}$ exists such that $b^{\prime}$ occurs in blocker $\left(a_{i}, w_{j}\right)$.

Let us check how many blockers can be covered by $(\beta)$, for all $1 \leq i \neq j \leq q$. The number of different $b^{\prime}$ is by Fact 6.1 at most by $q(r+1)$, and thus the number of different $b$ is at most $q(r+1)(2 r+1)$, as $b$ must be within distance $r$ to $b^{\prime}$. Hence, at most $q(r+1)(2 r+1) \cdot \ell$ many blocker $\left(a_{i}, w_{j}\right)$ can be covered by $(\beta)$; all others must be covered by $(\alpha)$. It follows that

$$
\left|\bigcup_{i=1}^{q} B\left(a_{i}\right) \cap \operatorname{freelit}(V)\right| \geq \frac{t(H) \cdot q}{r}-2 \cdot q-q(r+1)(2 r+1) \cdot \ell .
$$

Thus, we obtain the inequation

$$
q \cdot\left(\frac{t(H)}{r}-c_{0}\right) \leq \mid \text { freelit }(V) \mid,
$$

where $c_{0}=(2+(r+1)(2 r+1) \cdot \ell)$ depends only on $\Phi$. 
On the other hand, assume w.l.o.g. that the $\delta \in \Delta$ we have chosen is such that $q=\mid$ wit $_{r}(\delta, V) \mid$ is maximal over all clauses $\delta \in \Delta$. Then,

$$
|\operatorname{freelit}(V)| \leq|\Delta| \cdot q \cdot \ell .
$$

From (8) and (9), we derive that for maximal $q$, we have

$$
\frac{t(H) \cdot q}{r}-c_{0} \leq|\Delta| \cdot \ell
$$

whence

$$
t(H) \cdot q-c_{0} \cdot r \leq|\Delta| \cdot \ell \cdot r
$$

Observe that $H$ is $r^{\prime}$-uniform for some $r^{\prime} \leq r$. If $q>c_{r^{\prime}}$, then $|H|>c_{r^{\prime}}$, and since $H$ is $r^{\prime}$-uniform, by Corollary 4.4, we have $t(H)>\log |H|=\log q$. From this and (10) we obtain $(\log q)-c_{0} \cdot r \leq|\Delta| \cdot \ell \cdot r$, and thus $\log q \leq r \cdot\left(c_{0}+|\Delta| \cdot \ell\right)$, from which it follows that

$$
q \leq 2^{r \cdot\left(c_{0}+|\Delta| \cdot \ell\right)} .
$$

Let $k_{0}=\max \left\{c_{r}, 2^{r \cdot\left(c_{0}+|\Delta| \cdot \ell\right)}\right\}$ and $K=k_{0} \cdot|\Delta|$. Then, $q \leq k_{0}$, and both $k_{0}$ and $K$ are constants only depending on formula $\Phi$.

Since $q$ is maximal, we have

$$
\mid \text { wit }_{r}(V)\left|\leq \sum_{\gamma \in \Delta}\right| \text { wit }_{r}(\gamma, V)|\leq| \Delta \mid \cdot q \leq k_{0} \cdot \Delta=K,
$$

i.e., $\mid$ wit $_{r}(V) \mid \leq K$. This shows that $\Phi$ is bounded, which proves the theorem.

\section{$7 \operatorname{ESO}\left(\exists^{*} \forall \exists^{*}\right)$ is Regular}

The goal of this section is to finally show that the full class $\operatorname{ESO}\left(\exists^{*} \forall \exists^{*}\right)$ is regular. To this aim, we first define some automata-theoretic concepts.

Definition 7.1 A simple nondeterministic transducer $(S N T)$ is a tuple $T=\left(A, B, Q, E, q_{i n}, Q_{a}\right)$, where

- $A$ and $B$ are finite alphabets, called the input alphabet and the output alphabet of $T$, respectively;

- $Q$ is a finite set of states;

- $E \subseteq Q \times A \times B \times Q$ is a finite set of transitions, such that for each letter $a \in A$ and each state $q \in Q$, there exists some letter $b \in B$ and a state $q^{\prime} \in Q$ such that the tuple $\left(q, a, b, q^{\prime}\right) \in E$.

- $q_{\text {in }} \in Q$ is the initial state.

- $Q_{a} \subseteq Q$ is the set of accepting states.

A run $\overline{\mathrm{R}}$ of $T$ on input string $W \in A^{*}$ is a sequence of length $|W|$ of tuples $t_{i}=\left(q_{i}, a_{i}, b_{i}, q_{i}^{\prime}\right) \in E$, $1 \leq i \leq|W|$, such that the following conditions are satisfied: 
- $q_{1}=q_{i n}$

- for $1 \leq i \leq|W|, a_{i}=W_{i}$ is the $i$-th letter of $W$,

- for $1 \leq i \leq|W|-1, q_{i+1}=q_{i}^{\prime}$,

- $q_{|W|}^{\prime} \in Q_{a}$.

The output string $\overline{\mathrm{R}}(W)$ of such a run consists of the string $b_{1} b_{2} \ldots b_{|W|}$ in $B^{*}$.

The SNT $T$ associates with any input string $W \in A^{*}$ a set of output strings $T(W) \subseteq B^{*}$, namely $T(W)=\{r(W) \mid \overline{\mathrm{R}}$ is a run of $T$ on input $W\}$.

Definition 7.2 Let $L$ and $L^{\prime}$ be languages over alphabets $A$ and $B$, respectively. Then, $L$ is $S N T$ reducible to $L^{\prime}$, denoted by $L \preceq L^{\prime}$, iff there exists an SNT $T$ such that for each string $W \in A^{*}$ it holds that $W \in L$ iff $T(W) \cap L^{\prime} \neq \emptyset$.

The following proposition is a simple special case of more general results on transductions which can be found e.g. in [11, 4, 22] (in particular, see [22, Theorem 11.2, p. 276]).

Proposition 7.1 The class of regular languages is closed under $S N T$-reductions, i.e., if $L \preceq L^{\prime}$ and $L^{\prime}$ is regular, then also $L$ is regular.

Theorem 7.1 The class $\operatorname{ESO}\left(\exists^{*} \forall \exists^{*}\right)$ is regular.

Proof. Let $\Phi$ be an $\operatorname{ESO}\left(\exists^{*} \forall \exists^{*}\right)$ formula of the form $\exists \mathbf{P} \exists z_{1}, \ldots, z_{k} \forall x \exists y_{1}, \ldots, y_{r} . \varphi$ over an alphabet $A$, where $\varphi$ is quantifier-free.

Denote $C=\left\{\min , \max , z_{1}, \ldots, z_{k}\right\}$ and Terms $=C \cup\left\{x, y_{1}, \ldots, y_{r}\right\}$. Without loss of generality, we assume that $\varphi$ is a disjunction of complete types and that for all distinct $\chi, \xi \in$ Terms, each clause of $\varphi$ asserts $\chi \neq \xi$ and mentions all elements of Terms (cf. the proof of Theorem 5.4). Moreover, we assume w.l.o.g. that each clause $\delta$ of $\varphi$ contains for each term $\gamma \in$ Terms only one positive literal of the form $C_{a}(\gamma)$, where $C_{a}$ is in the signature (specifying the color of $x$ ). This literal is referred to as the color qualification of $x$ in $\delta$.

Let $L=\mathcal{L}(\Phi)$ be the language defined by $\Phi$ on $A$. Let $B=A \cup A \times C$ be an extension of the alphabet $A$. For any letter $e=(a, s) \in B$ such that $a \in A$ and $s \in C$, we will refer to $s$ as the label of $e$.

Let $T$ be an SNT with input alphabet $A$ and output alphabet $B$ operating as follows. For any string $W \in A^{*}, T$ rejects $W$ if $|W|<\mid$ Terms $\mid=k+r+3$; otherwise, $T$ has all runs $\overline{\mathrm{R}}$ which satisfy the following properties.

Denote the output for the letter $W_{i} \in A$ of $W$ by $\overline{\mathrm{R}}(W)_{i}, 1 \leq i \leq|W|$, and denote the output for $W$ by $\overline{\mathrm{R}}(W)$.

- The first letter $W_{1}$ of $W$ is transformed to $\overline{\mathrm{R}}(W)_{1}=\left(W_{1}, \min \right)$, i.e., the label $\min$ is attached to the first letter.

- The last letter $W_{|W|}$ of $W$ is transformed to $\overline{\mathrm{R}}(W)_{|W|}=\left(W_{|W|}, \max \right)$, i.e., the label max is attached to the last letter. 
- There are exactly $k$ distinct positions $1<i_{1}, \ldots, i_{k}<|W|$ such that $\overline{\mathrm{R}}(W)_{i_{1}}=\left(W_{i}, z_{1}\right), \overline{\mathrm{R}}(W)_{i_{2}}=$ $\left(W_{i}, z_{2}\right), \ldots, \overline{\mathrm{R}}(W)_{i_{k}}=\left(W_{i}, z_{k}\right)$. In other terms, exactly $k$ positions different from the first and the last of $W$ are labeled respectively with $z_{1}, \ldots, z_{k}$.

- For all positions $i \notin\left\{1, i_{1}, \ldots, i_{k},|W|\right\}$, it holds that $\overline{\mathrm{R}}(W)_{i}=W_{i}$.

The different nondeterministic runs of $T$ produce the set $T(W)$ consisting of all output strings fulfilling the above conditions. It is obvious that such a transducer $T$ exists.

Let $\Phi^{\prime}$ be the NF1 sentence (with possible occurrence of Succ) over alphabet $B$ and the corresponding signature of the form

$$
\exists \mathbf{P} \forall x \exists y_{1}, \ldots, y_{r} \exists z_{\text {min }}, z_{\text {max }}, z_{1}, \ldots, z_{k} \cdot \varphi^{\prime},
$$

where $\varphi^{\prime}$ is obtained from $\varphi$ as follows:

1. Eliminate the constant $\min$ by replacing $\min$ everywhere with the variable $z_{\min }$ and replace the color qualification $C_{a}(\min )$ of $\min$ in each clause $\delta$ by $C_{(a, \min )}\left(z_{\min }\right)$;

2. eliminate the constant $\max$ by replacing $\max$ everywhere with the variable $z_{\max }$ and replace the color qualification $C_{a}(\max )$ of $\max$ in each clause $\delta$ by $C_{(a, \max )}\left(z_{\max }\right)$;

3. adjust all color qualifications of the $z_{i}$ variables as follows. For each clause $\delta$, and each $1 \leq i \leq k$, replace the color qualification $C_{a}\left(z_{i}\right)$ in $\delta$ by $C_{\left(a, z_{i}\right)}\left(z_{i}\right)$;

4. transform the so obtained formula into a disjunction of complete types.

Let $L^{\prime}=\mathcal{L}\left(\Phi^{\prime}\right)$ be the language defined by $\Phi^{\prime}$. Since $\Phi^{\prime}$ is in NF1, $L^{\prime}$ is regular.

Claim 7.1 For each $W \in A^{*}, W \in L$ iff $T(W) \cap L^{\prime} \neq \emptyset$.

Proof of Claim. Assume first $W \in L$. Then, $W \models \Phi$, and hence there exist relations $\mathbf{P}$ and elements $a_{1}, \ldots, a_{k}$ in $W$ such that

$$
\left(W, \overline{\mathbf{R}}, a_{1} \ldots, a_{k}\right) \models \forall x \exists y_{1}, \ldots, y_{r} \varphi\left(z_{1}, \ldots, z_{k}\right) .
$$

Let $\overline{\mathrm{R}}$ be the run of $T$ which for $1 \leq i \leq k$ outputs $\overline{\mathrm{R}}(W)_{a_{i}}=\left(W_{i}, z_{i}\right)$. By the construction of $\Phi^{\prime}$, it is obvious that $\overline{\mathrm{R}}(W)$ satisfies $\Phi^{\prime}$, hence $\overline{\mathrm{R}}(W) \in L^{\prime}$. Since $\overline{\mathrm{R}}(W) \in T(W)$, it follows that $T(W) \cap L^{\prime} \neq \emptyset$.

Conversely, assume that $T(W) \cap L^{\prime} \neq \emptyset$. Then there is a run $\overline{\mathrm{R}}$ of $T$ such that $\overline{\mathrm{R}}(W) \in L^{\prime}$, and hence there exist relations $\mathbf{P}$ on $W$ such that

$$
(\overline{\mathrm{R}}(W), \mathbf{P}) \models \forall x \exists y_{1}, \ldots, y_{r} \exists z_{\min }, z_{\max }, z_{1}, \ldots, z_{k} \cdot \varphi^{\prime} .
$$

Let $a_{1}, \ldots, a_{k}$ be the positions of $\overline{\mathrm{R}}(W)$ marked $z_{1}, \ldots, z_{k}$, respectively. Moreover let $a_{\min }=1$, and $a_{\max }=|W|$. By the construction of $\Phi^{\prime}$, it is obvious that

$$
\left(\overline{\mathrm{R}}(W), \mathbf{P}, a_{\min }, a_{\max }, a_{1}, \ldots, a_{k}\right) \models \forall x \exists y_{1}, \ldots, y_{r} \cdot \varphi^{\prime}\left(z_{\min }, z_{\max }, z_{1}, \ldots, z_{k}\right),
$$

and thus

$$
\left(\overline{\mathrm{R}}(W), a_{\min }, a_{\max }\right) \models \exists \mathbf{P} \exists z_{1}, \ldots, z_{k} \forall x \exists y_{1}, \ldots, y_{r} \cdot \varphi^{\prime}\left(z_{\min }, z_{\max }\right),
$$


whence

$$
W \models \exists \mathbf{P} \exists z_{1}, \ldots, z_{k} \forall x \exists y_{1}, \ldots, y_{r} \cdot \varphi,
$$

and hence $W \models \Phi$, which proves that $W \in L . \quad \diamond$

In summary, we have shown that $L \preceq L^{\prime}$ via $T$. Since $L^{\prime}$ is regular, by Proposition 7.1, so is $L$.

Corollary 7.2 Over strings, $\operatorname{ESO}\left(\exists^{*} \forall \exists^{*}\right)$ is equivalent to MSO and existential MSO.

As already mentioned in the Introduction, equivalence of $\operatorname{ESO}\left(\exists^{*} \forall \exists^{*}\right)$ and existential MSO does not hold on finite ordered graphs. Indeed the property of disconnectivity of a finite ordered graph is expressible in MSO, while it is not expressible in $\operatorname{ESO}\left(\exists^{*} \forall \exists^{*}\right)$ [12]. On the other hand, both existential MSO and $\mathrm{ESO}\left(\exists^{*} \forall \exists^{*}\right)$ can express NP-complete graph properties.

\section{$8 \operatorname{ESO}\left(\exists^{*} \forall \forall\right)$ is Regular}

In this section, we show that $\operatorname{ESO}\left(\exists^{*} \forall \forall\right)$ is the second maximal standard ESO-prefix class which is regular. There are no further such classes, and thus we obtain a complete characterization of those ESO-prefix classes which are regular.

Theorem 8.1 Over strings, every $\operatorname{ESO}(\exists * \forall)$ sentence is equivalent to an MSO sentence.

Theorem 8.1 together with Büchi's Theorem (Proposition 2.1) implies the desired result.

Corollary 8.2 The class $\operatorname{ESO}\left(\exists^{*} \forall\right)$ is regular.

In the rest of this section, we prove Theorem 8.1 under simplifying assumptions. A compact full proof is given in the appendix.

Let $\Phi$ be a $\operatorname{ESO}\left(\exists^{*} \forall \forall\right)$ sentence $\exists \mathbf{R} \exists \mathbf{y} \forall x_{1}, x_{2} . \varphi$ where $\varphi$ is quantifier free.

Lemma 8.3 $\Phi$ is equivalent to a disjunction of $\operatorname{ESO}\left(\exists^{*} \forall \forall\right)$ sentences $\exists \mathbf{R} \exists \mathbf{y} \forall x_{1} x_{2} \varphi_{i}$ such that each $\varphi_{i}$ fixes (that is uniquely determines) the quantifier-free type of $\mathbf{y}$.

(A quantifier-free type of $\mathbf{y}$ is a complete type of $\mathbf{y}$ on $=$ and the relations of a string.)

Proof. Let $\psi_{1}(\mathbf{y}), \ldots, \psi_{m}(\mathbf{y})$ be the quantifier-free types of $\mathbf{y}$ consistent with $\varphi$. The desired $\varphi_{i}=\psi_{i} \wedge \varphi$.

In the rest of the proof, we assume, without loss of generality, that $\varphi$ fixes the quantifier-free type of $\mathbf{y}$, and that $\min$ and $\max$ do not occur in $\varphi$ (they can be defined using additional variables $y_{\min }$ and $y_{\max }$ in $\mathbf{y}$ and adding $\neg \operatorname{Succ}\left(x_{1}, y_{\min }\right)$ and $\neg S u c c\left(y_{\max }, x_{1}\right)$ to $\left.\varphi\right)$.

Let $y_{1}, \ldots, y_{n}$ be the constituents of $\mathbf{y}$. Without loss of generality, $\varphi$ implies that all $n+2$ individual variables take different values. Indeed, if $\varphi$ implies that two distinct constituents of $\mathbf{y}$ have the same values, then one of the constituents can be eliminated.

Without loss of generality, restrict attention to strings of length $\geq n+2$. If $\psi\left(\mathbf{y}, x_{1}, x_{2}\right)$ is quantifier free, let $\dot{\forall} x_{1} x_{2} \psi\left(x_{1}, x_{2}\right)$ assert that $\psi\left(\mathbf{y}, x_{1}, x_{2}\right)$ holds for all values $x_{1}, x_{2}$ such that 


$$
x_{1} \neq x_{2}, \quad \bigwedge_{i} x_{1} \neq y_{i}, \quad \bigwedge_{i} x_{2} \neq y_{i}
$$

Call this universal quantifier strict.

Lemma 8.4 $\Phi$ is equivalent to a sentence of form $\exists \mathbf{R} \exists \mathbf{y} \forall \dot{\forall} x_{1}, x_{2} \psi$ where $\psi$ is quantifier-free.

Proof. Replace $\forall x_{1} x_{2} \varphi\left(\mathbf{y}, x_{1}, x_{2}\right)$ with $\dot{\forall} x_{1} x_{2} \psi\left(\mathbf{y}, x_{1}, x_{2}\right)$, where $\psi$ is the conjunction of formulas

$$
\bigwedge_{i, j} \varphi\left(\mathbf{y}, y_{i}, y_{j}\right), \bigwedge_{i} \varphi\left(\mathbf{y}, x_{1}, y_{i}\right), \quad \bigwedge_{i} \varphi\left(\mathbf{y}, y_{i}, x_{2}\right), \quad \varphi\left(\mathbf{y}, x_{1}, x_{2}\right) .
$$

We therefore will assume that the universal quantifier in $\Phi$ is strict.

We illustrate the rest of the proof on the example where $\mathbf{R}$ contains only unary predicates and one binary predicate $E$. Thus $\Phi=\exists \mathbf{U} \exists E \exists \mathbf{y} \dot{\forall} x_{1} x_{2} \varphi_{1}$ where $\mathbf{U}$ is a tuple of unary predicates.

Lemma 8.5 Without loss of generality, $\varphi$ contains no E-atoms with at most one universal variable.

Proof. We illustrate the proof on the example where $\mathbf{y}$ contains only one constituent $y$. In that case, there are seven possible $E$-atoms with at most one universal variable:

$$
E\left(x_{1}, x_{1}\right), E\left(x_{2}, x_{2}\right), E\left(x_{1}, y\right), E\left(x_{2}, y\right), E\left(y, x_{1}\right), E\left(y, x_{2}\right), E(y, y)
$$

Using fresh unary predicate variables $E^{*, *}, E^{*, y}, E^{y, *}$ and a nullary predicate variable $E^{y, y}$ replace the seven atoms in $\varphi$ with atoms

$$
E^{*, *}\left(x_{1}\right), \quad E^{*, *}\left(x_{2}\right), \quad E^{*, y}\left(x_{1}\right), \quad E^{*, y}\left(x_{2}\right), \quad E^{y, *}\left(x_{1}\right), \quad E^{y, *}\left(x_{2}\right), \quad E^{y, y}
$$

respectively. Let $\psi$ be the resulting formula and let

$$
\Psi=\exists \mathbf{U} \exists E \exists \mathbf{P} \exists y \dot{\forall} x_{1} x_{2} \psi
$$

We check that $\Psi$ is equivalent to $\Phi$. First suppose that a string $W$ models $\Phi$ and fix the values of all existential variables so that the expanded structure $W^{*}$ models $\dot{\forall} x_{1} x_{2} \psi$. Let $b$ be the value of $y$. Set

$$
\begin{aligned}
E^{*, *}(x) & :=E(x, x) \\
E^{*, y}(x) & :=E(x, b) \\
E^{y, *}(x) & :=E(b, x) \\
E^{y, y} & :=E(b, b)
\end{aligned}
$$

Clearly $W^{*} \models \exists P Q \exists E \exists \mathbf{y} \forall x_{1} x_{2} \psi$ and therefore $W \models \Psi$.

Second suppose that a string $W$ models $\Psi$ and fix the values of all existential variables so that the expanded structure $W^{*}$ models $\dot{\forall} x_{1} x_{2} \varphi$. Let $b$ be the value of $y$. Notice that $\psi$ says nothing about $E$ on pairs $(x, x),(b, x),(x, b)$. Redefine $E$ on these pairs with respect to the following recipe:

$$
\begin{aligned}
E(x, x) & :=E^{*, *}(x) \\
E(x, b) & :=E^{*, y}(x) \\
E(b, x) & :=E^{y, *}(x) \\
E(b, b) & :=E^{y, y}
\end{aligned}
$$

The modified $W^{*}$ satisfies $\varphi$. Hence $W \models \Phi$. 
Remark 3 The mnemonic names $E^{*, *}, E^{*, y}$ and $E^{y, *}$ ease transition to the case when $\mathbf{y}$ has several constituents or $\mathbf{R}$ has several predicates of arity $>1$. If $\mathbf{y}$ has two constituents $y_{1}, y_{2}$ but the binary predicate $E$ still is the only non-unary predicate, the new unary predicates are

$$
E^{*, *}, E^{*, y_{1}}, E^{*, y_{2}}, E^{y_{1}, *}, E^{y_{2}, *}
$$

and the new nullary predicates

$$
E^{y_{1}, y_{1}}, E^{y_{1}, y_{2}}, E^{y_{2}, y_{1}}, E^{y_{2}, y_{2}} .
$$

If $\mathbf{y}$ has only one constituent $y$ and a ternary predicate $R$ is the only non-unary predicate in $\mathbf{R}$, then the new unary predicates are

$$
R^{*, *, *}, R^{y, *, *}, R^{*, y, *}, R^{*, *, y}, R^{y, y, *}, R^{y, *, y}, R^{*, y, y}
$$

and the only new nullary predicate is $R^{y, y, y}$.

In the rest of the proof, we assume that $\varphi$ does not contain $E$-atoms with at most one universal variable. The only $E$-atoms that can appear in $\varphi$ are $E\left(x_{1}, x_{2}\right)$ and $E\left(x_{2}, x_{1}\right)$.

Let $P$ and $Q$ be fresh nullary predicate variables, and let $\varphi^{\prime}\left(\mathbf{y}, x_{1}, x_{2}\right)$ (respectively $\left.\varphi^{\prime \prime}\left(\mathbf{y}, x_{1}, x_{2}\right)\right)$ be obtained from $\varphi\left(\mathbf{y}, x_{1}, x_{2}\right)$ be replacing $E\left(x_{1}, x_{2}\right)$ with $P$ (respectively $Q$ ) and replacing $E\left(x_{2}, x_{1}\right)$ with $Q$ (respectively $P$ ).

Lemma 8.6 The formula $\exists E \dot{\forall} x_{1} x_{2} \varphi\left(\mathbf{y}, x_{1}, x_{2}\right)$ is logically equivalent to the MSO formula

$$
\dot{\forall} x_{1} x_{2} \exists P, Q\left[\left(\varphi^{\prime}\left(\mathbf{y}, x_{1}, x_{2}\right) \wedge \varphi^{\prime \prime}\left(\mathbf{y}, x_{2}, x_{1}\right)\right] .\right.
$$

Proof. Let $\alpha(\mathbf{y})=\dot{\forall} x_{1} x_{2} \varphi\left(\mathbf{y}, x_{1}, x_{2}\right)$ and $\beta(\mathbf{y})$ be the MSO formula above.

First we suppose that $(M, E) \models \alpha(\mathbf{b})$, where $M$ is any model. We check that $M \models \beta(\mathbf{b})$. Let $a_{1}, a_{2}$ be distinct elements of $M$ that do not occur in $\mathbf{b}$, and set $P:=E\left(a_{1}, a_{2}\right), Q:=E\left(a_{2}, a_{1}\right)$. Since $(M, E) \models \varphi\left(\mathbf{b}, a_{1}, a_{2}\right), M \models \varphi^{\prime}\left(\mathbf{b}, a_{1}, a_{2}\right)$. Since $(M, E) \models \varphi\left(\mathbf{b}, a_{2}, a_{1}\right), M=\varphi^{\prime \prime}\left(\mathbf{b}, a_{2}, a_{1}\right)$. Thus $M \vDash \exists P Q\left[\varphi^{\prime}\left(\mathbf{b}, a_{1}, a_{2}\right) \wedge \varphi^{\prime \prime}\left(\mathbf{b}, a_{1}, a_{2}\right)\right]$. Since $a_{1}, a_{2}$ were arbitrary distinct elements outside of $\mathbf{b}$, $M \models \beta(\mathbf{b})$.

Second suppose that $M \models \beta(\mathbf{b})$ and order the elements of $M$. For all elements $a_{1}<a_{2}$ in $M$ that do not occur in $\mathbf{b}$, choose (the values of) $P$ and $Q$ such that $M \models \varphi^{\prime}\left(\mathbf{b}, a_{1}, a_{2}\right) \wedge \varphi^{\prime \prime}\left(\mathbf{b}, a_{2}, a_{1}\right)$. Set $E\left(a_{1}, a_{2}\right):=P$ and $E\left(a_{2}, a_{1}\right):=Q$. Define the remaining values of $E$ arbitrarily.

We check that $(M, E) \models \alpha(\mathbf{b})$. Indeed, let $a_{1}<a_{2}$ be distinct elements of $M$ outside of $\mathbf{b}$, and set $P:=E\left(a_{1}, a_{2}\right), Q:=E\left(a_{2}, a_{1}\right)$. By the definition of $E, M \models \varphi^{\prime}\left(\mathbf{b}, a_{1}, a_{2}\right) \wedge \varphi^{\prime \prime}\left(\mathbf{b}, a_{2}, a_{1}\right)$. It follows that $(M, E)$ models both $\varphi\left(\mathbf{b}, a_{1}, a_{2}\right)$ and $\varphi\left(\mathbf{b}, a_{2}, a_{1}\right)$. Thus $(M, E) \models \alpha(\mathbf{b})$. Hence $M \models \exists E \alpha(\mathbf{b})$.

It follows that the sentence $\Phi$ is equivalent to an MSO sentence. This establishes Theorem 8.1 (see appendix for a full proof).

By Corollary 8.2 and the results in the previous sections, we thus obtain the following exhaustive characterization of the regular ESO-prefix classes.

Theorem 8.7 (i) $\operatorname{ESO}\left(\exists^{*} \forall \exists^{*}\right)$ and $\operatorname{ESO}\left(\exists^{*} \forall \forall\right)$ are alle the maximal regular standard $\mathrm{ESO}$-prefix classes.

(ii) The unique maximal (general) regular ESO-prefix class is given by $\operatorname{ESO}\left(\exists^{*} \forall \exists^{*}\right) \cup \operatorname{ESO}\left(\exists^{*} \forall \forall\right)=$ $\operatorname{ESO}\left(\exists^{*} \forall\left(\forall \cup \exists^{*}\right)\right)$.

(iii) There are three minimal nonregular ESO-prefix classes, which are the standard ESO-prefix classes $\operatorname{ESO}(\forall \forall \forall), \operatorname{ESO}(\forall \forall \exists)$, and $\operatorname{ESO}(\forall \exists \forall)$. 


\section{A Dichotomy Theorem for Model Checking}

In this section, we establish a result which is rather unexpected: For any ESO-prefix class, model checking is either possible by a DFA (and thus in constant space), or it is NP-complete. This shows that there is a (provably) huge gap in the computational complexity of different ESO-prefix classes.

Theorem 9.1 Model checking for $\operatorname{ESO}(Q)$ (i.e., given a string $W$, decide whether $W \models \Phi$ where $\Phi$ is fixed) is NP-complete, for every $Q \in\{\forall \forall \forall, \forall \forall \exists, \forall \exists \forall\}$. Moreover, NP-hardness holds for sentences whose list of second-order variables consists of a single binary predicate variable.

Proof. Clearly, the problem is in NP. For the hardness part, we show that SAT can be reduced to model checking for $\Sigma_{1}^{1}(Q)$.

We first show this for $Q=\forall \forall \forall$, and then by adaptations of the proof for the other prefixes. For making the proof more intelligible, we first show that SAT is expressible by formulas with monadic and two predicate variables $R, R^{\prime}$; later, we will show how to get rid of all predicate variables except for $R$.

We choose a string encoding of SAT instances, which are collections of clauses $\mathcal{C}=\left\{C_{1}, \ldots C_{m}\right\}$ on propositional variables $p_{1}, \ldots, p_{n}$, as follows.

The alphabet is $A=\{0,1,+,-,[],,()$,$\} . We encode the variables p_{i}, 1 \leq i \leq n$, by binary strings of length $\lceil\log n\rceil$. Each string $\left\langle p_{i}\right\rangle$ encoding $p_{i}$ is enclosed by parentheses ' (',')', The polarity of a literal $p_{i} / \neg p_{i}$ is represented by the letters ' + ' or '-', respectively, which immediately follows the closing parenthesis ') ' of the encoding of $p_{i}$. A clause is encoded as a sequence of literals which is enclosed in square brackets ' [',']'. We assume w.l.o.g. that $\mathcal{C} \neq \emptyset$ and that each clause $C_{i} \in \mathcal{C}$ contains at least one literal.

For example, the clause set $\mathcal{C}=\{\{p, q, \neg r\},\{\neg p, \neg q, r\}\}$ is encoded by the following string:

$$
[(00)+(01)+(10)-][(00)-(01)-(10)+] .
$$

Here, the propositional variables $p, q, r$ are encoded by the binary strings $00,01,10$, respectively.

This encoding is somewhat redundant but very intuitive. It is evident that such an encoding can be obtained from any standard representation of SAT in logspace.

In what follows, we will use the formulas

$$
\begin{aligned}
\operatorname{eqcol}(x, y) & =\bigvee_{\ell \in A}\left(C_{\ell}(x) \wedge C_{\ell}(y)\right) \\
\operatorname{varenc}(x) & =C_{(}(x) \vee C_{0}(x) \vee C_{1}(x) \vee C_{)}(x)
\end{aligned}
$$

which state that the string has at positions $x$ and $y$ the same letter from $A$ and that $x$ is a letter of a variable encoding, respectively.

Consider the $\Sigma_{1}^{1}(\forall \forall \forall)$ formula

$$
\Phi=\exists G, V, R, R^{\prime} \forall x \forall y \forall z \cdot \varphi,
$$

where $G$ and $V$ are unary, $R^{\prime}$ and $R$ are binary, and $\varphi$ is the conjunction of the following quantifier-free formulas:

$$
\varphi_{G}=\varphi_{G, 1} \wedge \varphi_{G, 2} \wedge \varphi_{G, 3}
$$


where

$$
\begin{aligned}
\varphi_{G, 1}= & \left(C_{[}(x) \rightarrow \neg G(x)\right) \wedge\left(C_{]}(x) \rightarrow G(x)\right), \\
\varphi_{G, 2}= & \left(\operatorname{Succ}(x, y) \wedge \neg C_{[}(y) \wedge \neg C_{)}(y)\right) \rightarrow(G(y) \leftrightarrow G(x)), \\
\varphi_{G, 3}= & \left(C_{)}(y) \wedge \operatorname{Succ}(x, y) \wedge S u c c(y, z)\right) \rightarrow \\
& \left(G(y) \leftrightarrow\left[G(x) \vee\left(V(y) \wedge C_{+}(z)\right) \vee\left(\neg V(y) \wedge C_{-}(z)\right)\right]\right),
\end{aligned}
$$

and

$$
\begin{aligned}
\varphi_{V}= & \left(C_{)}(x) \wedge C_{)}(y) \wedge R(x, y)\right) \rightarrow(V(x) \leftrightarrow V(y)) \\
\varphi_{R}= & {[R(x, y) \rightarrow(\operatorname{eqcol}(x, y) \wedge \operatorname{varenc}(x))] \wedge } \\
& {\left[\left(C_{(}(x) \wedge C_{(}(y)\right) \rightarrow R(x, y)\right] \wedge } \\
& {\left[\left(\neg C_{(}(x) \wedge \operatorname{Succ}(z, x)\right) \rightarrow\left(R(x, y) \leftrightarrow\left(R^{\prime}(z, y) \wedge \operatorname{eqcol}(x, y)\right)\right)\right] } \\
\varphi_{R^{\prime}}= & S u c c(z, y) \rightarrow\left[R^{\prime}(x, y) \leftrightarrow\left(R(x, z) \wedge \neg C_{)}(z)\right)\right]
\end{aligned}
$$

The intuition behind this formula is as follows. The predicate $V$ assigns a truth value to each occurrence of a variable in the represented clause set $\mathcal{C}$, which is given by the value of $V$ at the closing parenthesis of this occurrence. The clause set $\mathcal{C}$ is satisfiable, precisely if there exists such a $V$ assigning every occurrence of the same variable the same truth value, such that every clause is satisfied. This property is checked by the use of $G, R$, and $R^{\prime}$.

The predicate $G$ is used for checking whether each clause $C \in \mathcal{C}$ is satisfied by the assignment $V$. To this end, the predicate $G$ is set to false at the ' [' marking the beginning of $C$, and set to true at the '] ' marking the end of $C$ by formula $\varphi_{G, 1}$; the formulas $\varphi_{G, 2}$ and $\varphi_{G, 3}$ propagate the value of $G$ from a position $x$ in the clause representation to the successor position $y$, where the value switches from false to true if $y$ marks the sign of a literal which is satisfied by $V$; the conjunct $\neg C_{\text {[ }}$ in $\varphi_{G}$, prohibits the transfer of $G$ from the end of $C$ to the beginning of the next clause, for which $G$ must be initialized to false.

The predicate $R$ is used to identify the closing parentheses ')' of the representations of occurrences of the same variables. For positions $x$ and $y$ at which the string $W$ has letter ') ', the predicate $R(x, y)$ is true precisely if $x$ and $y$ mark the end of the same variable name. This is used in the formula $\varphi_{V}$, which then simply states that $V$ assigns every occurrence of a variable $p$ in $\mathcal{C}$ the same truth value.

The purpose of the formulas $\varphi_{R}$ and $\varphi_{R^{\prime}}$ is to ensure that $R(x, y)$ has for positions $x$ and $y$ which mark the ends of occurrences of the same variable the desired meaning. This is accomplished in an inductive way. $R(x, y)$ intuitively expresses the following: $x$ and $y$ have the same color and must be part of the encodings $o_{x}=(\cdots)$ and $o_{y}=(\cdots)$ of variable occurrences in $\mathcal{C}$; moreover, $x$ and $y$ are at the same distance from the beginnings of these encodings. By reference to the predicate $R^{\prime}, R(x, y)$ furthermore expresses that these properties also hold for the pair $\left(x^{-}, y^{-}\right.$), where $x^{-}$(resp., $y^{-}$) is the predecessor of $x$ in $o_{x}$ (resp., of $y$ in $o_{y}$ ). The predicate $R^{\prime}$ is an auxiliary predicate since we may not introduce additional first-order variables, which are needed in the natural statement of the inductive property of $R$.

The formulas $\varphi_{R}$ and $\varphi_{R^{\prime}}$ thus effect that if $R(x, y)$ is true for positions $x$ and $y$ with letter ') ' in the string, then $x$ and $y$ mark the end of the same variable. 
We establish the following fact. Let for each $x \in W$ such that $W \models \operatorname{varenc}(x)$ be $b v(x)=\max \{y \mid$ $\left.y \leq x, W \models C_{(}(y)\right\}$ the closest ' (' in $W$ preceding or identical to $x$. Moreover, denote by reldist $(x)$ the distance between $b v(x)$ and $x$ and by $\operatorname{preds}^{v}(x)=\{y \in W \mid b v(x) \leq y<x\}$ the set of all elements $y$ preceding $x$ in the encoding of the variable to which $x$ contributes.

Fact 9.1 Suppose that for relations $R, R^{\prime}$ we have $\left(W, R, R^{\prime}\right) \models \forall x \forall y \forall z \cdot \varphi_{R} \wedge \varphi_{R^{\prime}}$. Then, for every elements $x, y \in W, R(x, y)$ holds iff

1. $W \models \operatorname{eqcol}(x, y)$,

2. $W \models \operatorname{varenc}(x)$,

3. $\operatorname{reldist}(x)=\operatorname{reldist}(y)$, and

4. for all $x^{\prime} \in \operatorname{preds}^{v}(x), y^{\prime} \in \operatorname{preds}^{v}(y)$ it holds that reldist $\left(x^{\prime}\right)=\operatorname{reldist}\left(y^{\prime}\right)$ implies eqcol $\left(x^{\prime}, y^{\prime}\right)$.

Proof. For every elements $x, y \in W$ such that $W \models \neg e q \operatorname{col}(x, y)$ or $W \models \neg \operatorname{varenc}(x)$, we clearly have $\neg R(x, y)$ by formula $\varphi_{R}$. Thus, it suffices to consider all elements $x, y \in W$ such that $W \models e q \operatorname{col}(x, y) \wedge$ $\operatorname{varenc}(x)$, (and thus $W \models \operatorname{varenc}(y)$ ).

For such $x, y$, we prove the claimed equivalence by induction on $n=\max \{\operatorname{reldist}(x), \operatorname{reldist}(y)\}$.

For $n=0$, it is easy to see that the equivalence holds.

Consider thus $n>0$. Then, we have reldist(x), reldist $(y)>0$ and $W \models \neg C_{(}(x)$. Suppose that $R(x, y)$ holds. Then, by part (14) of formula $\varphi_{R}$, for the predecessor $x^{-}$of $x$ in $W, R^{\prime}\left(x^{-}, y\right)$ holds (note that $x^{-}$must exist). By the formula $\varphi_{R^{\prime}}$, we have that for the predecessor $y^{-}$of $y$ in $W, R\left(x^{-}, y^{-}\right)$is true. From the induction hypothesis, we thus easily conclude that 3. and 4. hold if $R(x, y)$ holds.

Conversely, suppose that 1. -4 . hold. Then, by the induction hypothesis, we have that $R\left(x^{-}, y^{-}\right)$holds where $x^{-}$and $y^{-}$are as previously. Since clearly $\left.W \not \models C\right)\left(y^{-}\right)$, by $\varphi_{R^{\prime}}$ we have that $R^{\prime}\left(x^{-}, y\right)$ is true, and by part (14) of formula $\varphi_{R}$ we thus have that $R(x, y)$ is true. This concludes the induction step and thus the proof of Fact 9.1.

On the other hand, for every word $W$ encoding a SAT instance, we can define relations $R$ and $R^{\prime}$ such that $\left(W, R, R^{\prime}\right) \models \forall x, y, z \cdot \varphi_{R} \wedge \varphi_{R^{\prime}}$. Indeed, include in $R$ all tuples $(x, y)$ which satisfy 1. - 4. in Fact 9.1, and include in $R^{\prime}$ all tuples $\left(x, y^{+}\right)$such that $(x, y)$ is in $R, W \models S u c c\left(y, y^{+}\right)$, and $W \models \neg C_{)}(y)$. For these $R$ and $R^{\prime}$ we can check that $\left(W, R, R^{\prime}\right) \models \forall x, y, z \cdot \varphi_{R} \wedge \varphi_{R^{\prime}}$.

Claim 9.1 For a string $W$ encoding a clause set $\mathcal{C}$, it holds that $\mathcal{C}$ is satisfiable iff $W \models \Phi$.

Proof of claim. Suppose that $\mathcal{C}$ is satisfiable. Then, there exists an assignment $V$ of truth values to all occurrences of propositional atoms in $\mathcal{C}$ such that all occurrences of the same variables receive the same value and every clause is satisfied. We thus can readily define relations $V, G, R$ and $R^{\prime}$ such that $\left(W, G, V, R, R^{\prime}\right) \models \forall x \forall y \forall z \varphi$, and thus $W \models \Phi$; on the segment encoding a clause $C \in \mathcal{C}$ in $W, G$ is false until the end marker') ' of the first encoding of a variable in $C$ such that $V$ makes the corresponding literal true, and $G$ is true from there onwards.

On the other hand, suppose that $W \models \Phi$. Hence, there exist relations $G, V, R$, and $R^{\prime}$ such that $(W, G$, $\left.V, R, R^{\prime}\right) \models \forall x \forall y \forall z \varphi$. Define a truth assignment $\tau$ to the variables $p$ in $\mathcal{C}$ by $\tau(p)=V(x)$, where $x \in W$ is any end marker' ')' of the encoding of an occurrence of $p$ in $\mathcal{C}$. 
From Fact 9.1, it follows that if $R(x, y)$ holds for elements $x, y \in W$ such that $W \models C_{)}(x) \wedge C_{)}(y)$, then $x$ and $y$ mark the ends of the occurrences of the same variable. Hence, by formula $\varphi_{V}$, the end markers $x_{1}(p), \ldots, x_{k_{p}}(p)$ of the encodings of all occurrences of variable $p$ in $\mathcal{C}$ have the same value in $V$. From formula $\varphi_{G}$, it thus follows that for every clause $C \in \mathcal{C}$, there must exist a literal $L \in C$ such that $\tau$ makes $L$ true. Hence, $\mathcal{C}$ is satisfiable. This proves the claim.

This concludes the proof for the class $\operatorname{ESO}(Q)$ where $Q=\forall \forall \forall$. For the remaining two classes $(Q=\forall \forall \exists$, $Q=\forall \exists \forall)$, we slightly adjust $\Phi$. Let $C_{+}^{\prime}$ be a fresh monadic predicate variable, and define

$$
\varphi_{+}=\operatorname{Succ}(x, y) \rightarrow\left(\left(C_{+}^{\prime}(x) \leftrightarrow C_{+}(x)\right)\right.
$$

intuitively, $\varphi_{+}$states that $C_{+}^{\prime}$ is a left shift of $C_{+}$by one position in the string (where the value of $C_{+}^{\prime}(\max )$ is not specified).

Modify $\varphi$ now to a formula $\varphi^{\prime}$ as follows.

- add $\varphi_{+}$as a conjunct;

- replace $\varphi_{G, 3}$ by the following formula $\varphi_{G, 3}^{\prime}$ :

$$
\begin{aligned}
\left.\varphi_{G, 3}=(C)(y) \wedge S u c c(x, y)\right) \rightarrow \\
\\
{\left[G(y) \leftrightarrow\left(G(x) \vee\left(V(y) \wedge C_{+}^{\prime}(y)\right) \vee\left(\neg V(y) \wedge \neg C_{+}^{\prime}(y)\right)\right)\right] ; }
\end{aligned}
$$

- rewrite $\varphi_{R}$ to $\varphi_{R}^{\prime}$ by moving $\operatorname{Succ}(z, x)$ in the third conjunct from the premise to consequent, i.e.,

$$
\begin{aligned}
\varphi_{R}^{\prime}= & {[R(x, y) \rightarrow(\operatorname{eqcol}(x, y) \wedge \operatorname{varenc}(x))] \wedge } \\
& {\left[\left(C_{(}(x) \wedge C_{(}(y)\right) \rightarrow R(x, y)\right] \wedge } \\
& {\left[\left(\neg C_{(}(x) \wedge(x \neq \min )\right) \rightarrow\right.} \\
& \left.\left(\operatorname{Succ}(z, x) \wedge\left(R(x, y) \leftrightarrow\left(R^{\prime}(z, y) \wedge \operatorname{eqcol}(x, y)\right)\right)\right)\right] ;
\end{aligned}
$$

- and, replace $\varphi_{R^{\prime}}$ by the formula

$$
\varphi_{R^{\prime}}^{\prime}=(x \neq \min ) \rightarrow\left[\operatorname{Succ}(z, x) \wedge\left(R^{\prime}(y, x) \leftrightarrow\left(R(y, z) \wedge \neg C_{)}(z)\right)\right)\right] .
$$

Observe that in the formula $\varphi^{\prime}$, the variable $z$ only occurs in $\varphi_{R}^{\prime}$ and in $\varphi_{R^{\prime}}^{\prime}$.

Let

$$
\begin{aligned}
\Phi_{\forall \forall \exists} & =\exists C_{+}^{\prime}, G, V, R, R^{\prime} \forall x \forall y \exists z \cdot \varphi^{\prime}, \\
\Phi_{\forall \exists \forall} & =\exists C_{+}^{\prime}, G, V, R, R^{\prime} \forall x \exists z \forall y . \varphi^{\prime} .
\end{aligned}
$$

Then, for every string $W$ which encodes a clause set $\mathcal{C}$, it holds that

$$
W \models \Phi \quad \Leftrightarrow \quad W \models \Phi_{\forall \forall \exists} \quad \Leftrightarrow \quad W \models \Phi_{\forall \exists \forall}
$$


Indeed, the formula $\forall x \forall y \exists z \varphi^{\prime}(x, y, z)$ is true in an expanded structure $\left(W, C_{+}^{\prime}, G, V, R, R^{\prime}\right)$, iff for all elements $a, b \in W, \varphi^{\prime}(a, b, f(a))$ is satisfied, where $f(a)$ is the predecessor of $a$ in $W$, if $a \neq m i n$, and $f(\min )$ is arbitrary (observe that then $R(a, b)$ is false if $a=\min$ or $b=\min$ ). Therefore, the existential quantifier on $z$ only depends on $x$ and can be moved before $\forall y$.

Thus, NP-hardness of model-checking holds for the prefix-classes $\operatorname{ESO}(\forall \forall \forall), \operatorname{ESO}(\forall \exists \forall)$, and $\operatorname{ESO}(\forall \forall \exists)$.

To conclude the proof of the theorem, we show first how we can get rid of the binary predicate variable $R^{\prime}$, and then how we can eliminate all monadic predicate variables.

Getting rid of $R^{\prime}$. Recall that $\Phi=\exists G, V, R, R^{\prime} \forall x \forall y \forall z . \varphi$. For any relations $G, V, R, R^{\prime}$ on a string $W$ such that $\left(W, G, V, R, R^{\prime}\right) \models \forall x \forall y \forall z . \varphi$, we observe the following:

1. For each elements $a, b \in U(W), R(a, b)$ and $R^{\prime}(a, b)$ never hold simultaneously; therefore, there is enough "space" in the relation $R$ for packing the tuples of $R^{\prime}$ into $R$ and discarding $R^{\prime}$, provided we can distinguish both groups of tuples from each other.

2. From Fact 9.1, it follows, in particular, that for elements $a, b \in W, R(a, b)$ holds only if $\operatorname{reldist}(a) \equiv$ reldist $(b)(\bmod 2)$. On the other hand, as easily seen, $R^{\prime}(a, b)$ only holds if $\operatorname{reldist}(a) \not \equiv \operatorname{reldist}(b)$ (mod 2). This allows us to distinguish possible tuples in $R$ from possible tuples in $R^{\prime}$. Thus, if we set $R:=R \cup R^{\prime}$, any tuple $(a, b)$ of the new $R$ stems from the old $R$, if $\operatorname{reldist}(a) \equiv \operatorname{reldist}(b)(\bmod 2)$, and from $R^{\prime}$, if reldist $(a) \not \equiv$ reldist $(b)(\bmod 2)$.

3. The property $\operatorname{reldist}(x) \equiv \operatorname{reldist}(y)(\bmod 2)$ is clearly a regular property. It can be logically defined via a monadic predicate $P$ such that

$$
P(x) \longleftrightarrow(\operatorname{reldist}(x) \equiv 0(\bmod 2))
$$

In fact, if predicate $P$ is available, then $\operatorname{reldist}(x) \equiv \operatorname{reldist}(y)(\bmod 2)$ if and only if $P(x) \leftrightarrow P(y)$.

Based on these observations, it is clear that the following formula $\Phi^{*}$ is equivalent to $\Phi$ over SAT instances:

$$
\Phi^{*}=\exists P, G, V, R \forall x \forall y \forall z\left(\varphi_{P}(x, y) \wedge \varphi^{*}(x, y, z)\right),
$$

where $\varphi_{P}(x, y)$ defines the predicate $P$ by

$$
\varphi_{P}(x, y)=\left(C_{(}(x) \rightarrow P(x)\right) \wedge[(\operatorname{Succ}(x, y) \wedge \operatorname{varenc}(x) \wedge \operatorname{varenc}(y)) \rightarrow(P(y) \leftrightarrow \neg P(x))],
$$

and $\varphi^{*}$ results from $\varphi$ by replacing any atom $R(\chi, \xi)$ with $R(\chi, \xi) \wedge(P(\chi) \leftrightarrow P(\xi))$ and replacing any atom $R^{\prime}(\chi, \xi)$ with $R(\chi, \xi) \wedge \neg(P(\chi) \leftrightarrow P(\xi))$.

From the sentences $\Phi_{\forall \forall \exists}$ and $\Phi_{\forall \exists \forall}$, we can remove $R^{\prime}$ in an analogous manner, obtaining sentences $\Phi_{\forall \forall \forall}^{*}$ and $\Phi_{\forall \exists \forall}^{*}$.

Getting rid of monadic predicate variables. Note that the only monadic predicate variables in the formulas $\Phi^{*}, \Phi_{\forall \forall \exists}^{*}$, and $\Phi_{\forall \exists \forall}^{*}$ are $C_{+}^{\prime}, G, V$, and $P$. Moreover, for every choice of relations $(G, V, P, R)$ witnessing that $W \models \Phi^{*}$ (resp., $\left(C_{+}^{\prime}, G, V, P, R\right)$ witnessing that $W \models \Phi_{\forall \forall \exists}^{*}$ or $W \models \Phi_{\forall \exists \forall}^{*}$ ), it holds that min and $\max$ do not occur in any tuple of $R$, if we strip off possible such tuples arising from the packing of $R^{\prime}$ into $R$ by adding $R^{\prime}(x, y) \models \operatorname{varenc}(x) \wedge \operatorname{varenc}(y)$ in the very beginning. Thus, it is possible to pack the monadic predicates into $R$ as follows. 
Replace in $\Phi^{*}, \Phi_{\forall \forall \exists}^{*}$, and $\Phi_{\forall \exists \forall}^{*}$ uniformly $C_{+}^{\prime}(\chi)$ by $R(\min , \chi), G(\chi)$ by $R(\max , \chi), V(\chi)$ by $R(\chi, \min )$, and $P(\chi)$ by $R(\chi, \max )$. By doing this and some minor adjustments, we obtain formulas $\Phi^{\dagger}, \Phi_{\forall \forall \exists}^{\dagger}$, and $\Phi_{\forall \exists \forall}^{\dagger}$ which contain $R$ as single predicate variable and which are over SAT instances equivalent to $\Phi^{*}, \Phi_{\forall \forall \exists}^{*}$, and $\Phi_{\forall \exists \forall}^{*}$, respectively.

Corollary 9.2 Let $\mathcal{Q} \subseteq\{\exists, \forall\}^{*}$ such that $\mathcal{Q} \cap \exists^{*} \forall\left(\exists^{+} \forall\{\exists, \forall\}^{*} \cup \forall\{\exists, \forall\}^{+}\right) \neq \emptyset$. Then, model checking for $\operatorname{ESO}(\mathcal{Q})$ is NP-complete.

From Theorems 8.7 and 9.1 we obtain the following dichotomy theorem.

Theorem 9.3 (Dichotomy Theorem for Model Checking) Let $\mathcal{Q} \subseteq\{\forall, \exists\}^{*}$ be any prefix set. Then,

(i) either $\operatorname{ESO}(\mathcal{Q})$ expresses only regular languages, or $\operatorname{ESO}(\mathcal{Q})$ expresses some $\mathrm{NP}$-complete languages;

(ii) model-checking for $\operatorname{ESO}(\mathcal{Q})$ is either NP-complete, or is feasible by a finite-state automaton (and thus in constant space and linear time);

(iii) if $\operatorname{ESO}(\mathcal{Q})$ expresses any nonregular language, then it also expresses an NP-complete language.

Observe that this dichotomy is provably strict and does not, like other dichotomy theorems, e.g. Schaefer's result on SAT [44], rely on widely believed assumptions such as $\mathrm{P} \neq \mathrm{NP}$. Furthermore, not every ESOprefix class which expresses some NP-complete languages can express all of NP. In fact, there is a unique minimal ESO-prefix class which captures NP.

Proposition 9.1 The unique minimal ESO-prefix class capturing NP over strings is $\mathrm{ESO}\left(\forall^{*}\right)$.

Proof. (Sketch) As shown in [32,13], this class captures NP over arbitrary finite ordered structures. Any (syntactically) smaller class $C$ must have a constant bound $k$ on the number of universal FO-quantifiers. But then model-checking for $C$ is feasible in $\operatorname{NTIME}\left(n^{k}\right)$ on a RAM, since all "existential data" can be guessed nondeterministically. (On a TM, the exponent may be slightly higher but still constant because it may be necessary to check the consistency between several consecutive guesses.) By well-known time hierarchy theorems, it follows that $C$ cannot express all of NP.

\section{Finite Satisfiability}

In this section, we prove some results concerning the satisfiability of $\operatorname{ESO}(\mathcal{Q})$ sentences $\Phi$ over strings, i.e., deciding whether $\mathcal{L}(\Phi) \neq \emptyset$. Recall from the introduction that the satisfiability problem for $\operatorname{ESO}(\mathcal{Q})$ is a reformulation of the satisfiability problem for $\mathrm{FO}(\mathcal{Q})$.

It turns out that the separation of regular and nonregular ESO-prefix classes also gives us a precise characterization of those classes for which satisfiability over strings is decidable. In fact, the regular and the decidable classes coincide. 
Theorem 10.1 Let $\mathcal{Q}$ be any prefix set. Then, over finite strings, the satisfiability problem for $\operatorname{ESO}(\mathcal{Q})$ is decidable if and only if $\operatorname{ESO}(\mathcal{Q})$ is regular.

Proof. The proofs of Theorem 7.1 (regularity of $\operatorname{ESO}\left(\exists^{*} \forall \exists^{*}\right)$ ) and Theorem 8.1 (regularity of $\operatorname{ESO}\left(\exists^{*} \forall \forall\right)$ ) are constructive, in the sense that a finite state automaton recognizing $\mathcal{L}(\Phi)$ can be effectively constructed from a given sentence $\Phi$. Hence, from Theorem 8.7 and the fact that the emptiness problem of finite automata is decidable, it follows that for every regular class $\operatorname{ESO}(\mathcal{Q})$, satisfiability over finite strings is decidable.

Conversely, it suffices to show that satisfiability over finite strings is undecidable for all classes $\operatorname{ESO}(Q)$ where $Q \in\{\forall \forall \forall, \forall \forall \exists, \forall \exists \forall\}$. This can be established by a simple encoding of the question whether a given domino problem has a periodic solution, which is up to minor adaptations identical to the encoding of domino problems into formulas of the Kahr-Moore-Wang class in the proof of [5, Theorem 3.1.9]. For readers' convenience, we describe the reduction here.

A domino system is a triple $\mathcal{D}=\langle D, H, V\rangle$ where $D$ is a finite set, whose elements are called dominoes, and $H, V \subseteq D \times D$ are binary relations. $\mathcal{D}$ tiles $\mathbb{N}^{+} \times \mathbb{N}^{+}$, where $\mathbb{N}^{+}$denotes the positive integers, if there is a tiling $\tau: \mathbb{N}^{+} \times \mathbb{N}^{+} \rightarrow D$ such that, for each $i, j \in \mathbb{N}^{+}$, it holds that

(i) If $\tau(i, j)=d$ and $\tau(i+1, j)=d^{\prime}$, then $\left(d, d^{\prime}\right) \in H$; and

(ii) If $\tau(i, j)=d$ and $\tau(i, j+1)=d^{\prime}$, then $\left(d, d^{\prime}\right) \in V$.

A domino system admits a periodic tiling of $\mathbb{N}^{+} \times \mathbb{N}^{+}$, if there are integers $h, v \in \mathbb{N}^{+}$such that for all $i, j \in \mathbb{N}^{+}, \tau(i, j)=\tau(i+h, j)$ and $\tau(i, j)=\tau(i, j+v)$. We note the following lemma.

Lemma 10.2 Whether a given domino system $\mathcal{D}$ admits a periodic tiling of $\mathbb{N}^{+} \times \mathbb{N}^{+}$is undecidable.

This is an immediate consequence of the stronger result that the classes of domino systems which do not admit tilings of $\mathbb{N}^{+} \times \mathbb{N}^{+}$and which admit periodic tilings of $\mathbb{N}^{+} \times \mathbb{N}^{+}$are recursively inseparable (see [5, Theorem 3.1.7]).

We describe the encoding of domino systems into formulas from $\operatorname{ESO}(\forall \exists \forall)$; for the other classes, it is similar. For each domino $d \in D$, use a binary predicate variable $P_{d}$, and let $\mathbf{P}$ denote the tuples of all these variables. Define the formula $\Phi$ as $\exists \mathbf{P} \forall x \exists x^{\prime} \forall y . \varphi\left(x, x^{\prime}, y\right)$, where

$$
\begin{aligned}
\varphi\left(x, x^{\prime}, y\right)= & \varphi_{s}\left(x, x^{\prime}\right) \wedge \bigwedge_{d \neq d^{\prime}} \neg\left(P_{d}(x, y) \wedge P_{d^{\prime}}(x, y)\right) \wedge \\
& \bigvee_{\left(d, d^{\prime}\right) \in H}\left(P_{d}(x, y) \wedge P_{d^{\prime}}\left(x^{\prime}, y\right)\right) \wedge \bigvee_{\left(d, d^{\prime}\right) \in V}\left(P_{d}(y, x) \wedge P_{d^{\prime}}\left(y, x^{\prime}\right)\right)
\end{aligned}
$$

and

$$
\varphi_{s}\left(x, x^{\prime}\right)=\operatorname{Succ}\left(x, x^{\prime}\right) \vee\left(x=\max \wedge x^{\prime}=\min \right)
$$

Intuitively, the formula $\varphi_{s}$ expands a given finite string periodically, by considering the first position in the string as the successor of the last position. The second conjunct of $\varphi$ states that at most one domino can be at any position, while the last two conjuncts express that some domino must be there and the tiling is admissible.

It holds that $\Phi$ is satisfiable on finite strings over any fixed alphabet $\mathrm{A}$, if and only if $\mathcal{D}$ admits a periodic tiling of $\mathbb{N}^{+} \times \mathbb{N}^{+}$. 
Indeed, if $\tau$ is a periodic tiling of $\mathbb{N}^{+} \times \mathbb{N}^{+}$with horizontal and vertical periods $h$ and $v$, respectively, then consider any string $W$ on $A$ whose length $|W|$ is the least common multiple of $h$ and $v$. Define for all $P_{d}$ in $\mathbf{P}$ relations on $W$ by $P_{d}(i, j) \leftrightarrow \tau(i, j)=d$. Then, $(W, \mathbf{P}) \models \forall x \exists x^{\prime} \forall y . \varphi\left(x, x^{\prime}, y\right)$, and hence $\Phi$ is satisfiable on finite strings over $A$.

Conversely, if for relations $\mathbf{P}$ on a string $W$ over $A$ it holds that $(W, \mathbf{P}) \models \forall x \exists x^{\prime} \forall y . \varphi\left(x, x^{\prime}, y\right)$, then the map $\tau: \mathbb{N}^{+} \times \mathbb{N}^{+} \rightarrow D$ defined by $\tau(i, j)=d$ iff $(W, \mathbf{P}) \models P_{d}\left(i^{\prime}, j^{\prime}\right)$, where $i^{\prime}=i-1(\bmod |W|)+1$ and $j^{\prime}=j-1(\bmod |W|)+1$, is a periodic tiling of $\mathbb{N}^{+} \times \mathbb{N}^{+}$.

From this theorem we immediately get a corollary on the finite satisfiability of first order formulas over strings. Note that, as already mentioned in the introduction, in the context of satisfiability, $\operatorname{FO}(\mathcal{Q})$ formulas may contain free predicate variables besides those occurring in the signature of the input string (i.e., $S u c c$, $C_{a}$, etc.).

\section{Corollary 10.3 Over finite strings, for any prefix set $\mathcal{Q}$ either}

$(i)$ the satisfiability problem for $F O(\mathcal{Q})$ is decidable and $\operatorname{ESO}(\mathcal{Q})$ defines only regular languages, or

$($ ii) the satisfiability problem for $F O(\mathcal{Q})$ is undecidable and $\operatorname{ESO}(\mathcal{Q})$ defines some NP-complete language.

\section{Capturing REG and Closure under Complementation}

In this section, we investigate ESO-prefix classes which capture the class of regular languages, and which are closed under complementation.

The following lemma is well-known; for an illustrative proof, see e.g. [27].

Lemma 11.1 Every formula $\exists \mathbf{R} \exists y_{1}, \ldots, y_{k} . \varphi$, where $\varphi$ is quantifier-free, is equivalent to a formula $\exists y_{1}, \ldots, y_{k} . \psi$, where $\psi$ is quantifier-free.

A similar but weaker lemma holds if we allow one universal FO quantifier before the quantifier-free part.

Lemma 11.2 Over strings, every formula $\Phi=\exists \mathbf{R} \exists y_{1}, \ldots, y_{k} \forall x . \varphi$, where $k \geq 0$ and $\varphi$ is quantifier-free, is equivalent to a finite disjunction of first-order formulas $\exists y_{1}, \ldots, y_{k} \forall x . \psi$, where $\psi$ is quantifier-free.

Proof. (Sketch) The proof follows the lines of the proof of Theorem 8.1. $\Phi$ can be assumed to be a disjunction of formulas $\Phi^{\prime}=\exists \mathbf{R} \exists y_{1}, \ldots, y_{k^{\prime}} \forall x \varphi^{\prime}$, where $k^{\prime} \leq k$ and $\varphi^{\prime}$ fixes the quantifier-free type of the variables $y_{i}$ such that they are all different from each other and from $\min$ and $\max$. It suffices to prove the claim for a formula $\Phi$ of this form to obtain the result.

Predicates $R \in \mathbf{R}$ of arity $>1$ can be replaced in $\Phi$ by monadic predicates, so that we may assume that all predicates in $\Phi$ have arity $\leq 1$. Moreover, the quantifier-free part of $\Phi$ can be rewritten to a DNF $\bigvee_{j} \delta_{j}$ of types $\delta_{j}$ each of which extends the quantifier-free type of the variables $y_{i}$ by fixing the location of $x$ w.r.t. the variables $y_{i}$ and $\min$ and $\max$ such that if $x=y_{i}$ for some $y_{i}$ (resp., $x=\min , x=\max$ ) occurs in $\delta$, then $x$ does not occur in any other literal of $\delta$.

Remove then all clauses $\delta_{i}$ which contain an opposite pair of literals, remove from the remaining types all literals $R(x), \neg R(x)$ where $R$ is any (monadic) predicate from $\mathbf{R}$, and remove atoms $R(c)$ where $c \in$ 
$\left\{\min , \max , y_{1}, \ldots, y_{k}\right\}$ by using fresh nullary predicate variables. It is not hard to see that the resulting formula (which contains no predicate variables of arity $>0$ ) is equivalent to a disjunction of formulas $\exists y_{1}, \ldots, y_{k} \forall x . \psi$, where $\psi$ is quantifier-free. This proves the lemma.

Theorem 11.3 Over strings, every language expressible in $\operatorname{ESO}\left(\exists^{*} \forall\right)$ is expressible in $\mathrm{FO}\left(\exists^{*} \forall\right)$.

Proof. By Lemma 11.2, it remains to show that any disjunction $\varphi=\bigvee_{i=1}^{k} \varphi_{i}$ of FO sentences $\varphi_{i}=$ $\exists \mathbf{y}_{i} \forall x . \psi_{i}$, where $\psi_{i}$ is quantifier-free, is over strings equivalent to some $\operatorname{FO}\left(\exists^{*} \forall\right)$ sentence $\psi$. Let $\mathbf{z}=$ $z_{1}, \ldots, z_{k}$ be fresh FO variables, and suppose the $\mathbf{y}_{i}$ are pairwise disjoint; the desired $\psi$ is

$$
\psi=\exists \mathbf{z} \exists \mathbf{y}_{1} \ldots \exists \mathbf{y}_{k} \forall x\left[\bigvee_{i} \psi_{i} \wedge \bigvee_{i}\left(z_{i}=\min \right) \wedge \bigwedge_{i}\left(\left(\min \neq \max \wedge z_{i}=\min \right) \rightarrow \psi_{i}\right)\right]
$$

Intuitively, each $z_{i}$ serves as a switch; if $\min \neq \max$ and $z_{i}=\min$, then the formula $\varphi_{i}$ is selected as a witness for the truth of $\varphi$. This is checked by the right conjunct of $\psi$. The case where $\min =\max$ is trivial.

By exploiting the previous lemmata, the following proposition is easily proved.

Proposition 11.1 Let $A=\{a, b\}$. Then, (i) $L_{1}=a^{*}$ is not expressible in $\operatorname{ESO}\left(\exists^{*}\right)$; (ii) $L_{2}=a^{*} b^{*}$ is not expressible in $\operatorname{ESO}\left(\exists^{*} \forall\right)$; (iii) $L_{3}=\{a, b\}^{+}\{b a, a b\}\{a, b\}^{+}$is not expressible in $\operatorname{ESO}(\{\exists, \forall\})$.

Proof. Lemma 11.1, part $(i)$ is easy; suppose a formula $\exists \mathbf{y} \cdot \varphi(\mathbf{y})$, where $\varphi$ is quantifier-free, expresses $L_{1}$. Then, for every $W \in a^{*}$, there is some tuple a for $\mathbf{y}$ on $W$ such that $W \models \varphi(\mathbf{a})$. For $W$ large enough, choose an element $b$ in $W$ which is not coincident and not adjacent to $\min , \max$ and any element in $\mathbf{a}$, and change the color of $x$. The resulting string $W^{\prime} \models \varphi(\mathbf{a})$; this contradicts that $\exists \mathbf{y} \varphi(\mathbf{y})$ expresses $L_{1}$.

Part (ii) is shown analogously. Suppose $L_{2}$ is expressible in $\operatorname{ESO}\left(\exists^{*} \forall\right)$. Then, from Lemma 11.2, it follows that $L_{2}$ is expressed by a finite disjunction $\varphi=\bigvee_{i=1}^{d} \varphi_{i}$ of formulas $\varphi_{i}=\exists y_{1}, \ldots, y_{k} \forall x \psi_{i}$, for some $k \geq 0$, where each $\varphi_{i}$ is quantifier-free.

Hence, for any string of form $W=a^{n} b^{n}$, there is some $\varphi_{i}$ such that $W \models \varphi_{i}$, i.e., there are elements $a_{1}, \ldots, a_{k}$ in $W$ such that $\left(W, a_{1}, \ldots, a_{k}\right) \models \forall x \psi_{i}$. For $W$ large enough (choose $\left.n>3 k+2\right)$, there are elements $x_{a}$ and $x_{b}$ of colors $C_{a}$ and $C_{b}$ in $W$, respectively, such that $x_{a}$ and $x_{b}$ are not adjacent or coincident to any of $\min , a_{1}, \ldots, a_{k}, \max$. Exchange the colors of $x_{a}$ and $x_{b}$, and let $W^{\prime}$ be the resulting string. It is easy to see that $\left(W^{\prime}, a_{1}, \ldots, a_{k}\right) \models \forall x . \psi_{i}$, and thus $W^{\prime} \models \varphi$. This contradicts that $\varphi$ expresses $L_{2}$.

To prove part $(i i i)$, suppose first $L_{3}$ is expressed in $\operatorname{ESO}(\exists)$. By Lemma 11.1, $L_{3}$ is expressed by a sentence $\exists x \varphi(x)$, where $\varphi$ is quantifier-free. Consider the string $W=a a b a$, which is in $L_{3}$; it is easily seen that if $W \models \exists x \varphi(x)$, then either $a^{4} \models \exists x \varphi(x)$ or $a b^{2} a \models \exists x \varphi(x)$; this contradicts that $\exists x . \varphi(x)$ expresses $L_{3}$.

Suppose then $L_{3}$ is expressible in $\operatorname{ESO}(\forall)$. By Lemma 11.2, $L_{3}$ is expressed by a disjunction $\varphi=$ $\bigvee_{i=1}^{n} \forall x \varphi_{i}(x)$ of universal formulas $\forall x \varphi_{i}(x)$, where $\varphi_{i}(x)$ is quantifier-free. Consider $W=a^{2} b a a^{2}$. Then, $W \models \forall x \varphi_{i}(x)$ for some $i$. Change the color of the third letter to $C_{a}$. Then, for the resulting $W^{\prime}=a^{6}$, we have $W^{\prime} \models \forall x \varphi_{i}(x)$, since the third and fourth letter of $W^{\prime}$ are indiscernible for $\varphi_{i}$ and $\varphi_{i}$ is true for the fourth letter of $W$. This contradicts that $\varphi$ expresses $L_{3}$.

We thus establish the following result. 
Theorem 11.4 Let $\mathcal{Q}$ be any nontrivial prefix set such that $\operatorname{ESO}(\mathcal{Q})$ is regular. Then, the following statements are equivalent:

(i) $\operatorname{ESO}(\mathcal{Q})$ is closed under complementation;

(ii) $\operatorname{ESO}(\mathcal{Q})$ captures REG;

(iii) $\mathcal{Q} \cap \exists^{*} \forall\{\forall, \exists\}^{+} \neq \emptyset$.

Proof. $\quad(i) \Rightarrow(i i) \quad$ Suppose that $\mathcal{Q}$ is closed under complementation. By Proposition 2.2, for establishing (ii) it suffices to show that $\mathcal{Q}$ contains some prefix $Q$ which contains $\forall \exists$ or $\forall \forall$ as a substring. We show that such a $Q$ must exist in $\mathcal{Q}$.

Suppose first that $\mathcal{Q}$ contains any prefix $Q^{\prime}$ such that $\left|Q^{\prime}\right|>1$. Observe that the complement $\overline{L_{2}}=$ $\{a, b\}^{*} b a\{a, b\}^{*}$ of the language $L_{2}$ in part (ii) of Proposition 11.1 is clearly expressible both in $\operatorname{ESO}(\exists \exists)$ and in $\operatorname{ESO}(\exists \forall)$, while $L_{2}$ is neither expressible in $\operatorname{ESO}(\exists \exists)$ nor in $\operatorname{ESO}(\exists \forall)$. Since $\mathcal{Q}$ is closed under complementation, it follows that in this case, $\mathcal{Q}$ contains some $Q$ having $\forall \exists$ or $\forall \forall$ as substring.

Suppose now that every prefix $Q \in \mathcal{Q}$ has length $\leq 1$. We derive a contradiction. Since $\mathcal{Q}$ is nontrivial, it follows that either $\exists$ or $\forall$ must belong to $\mathcal{Q}$, Suppose $\forall \in \mathcal{Q}$. Since the complement $\overline{L_{3}}=\{\lambda, a, b\} \cup$ $\{a, b\}\left(a^{*} \cup b^{*}\right)\{a, b\}$ of the language $L_{3}$ in part (iii) of Proposition 11.1 is easily expressed in $\operatorname{ESO}(\forall)$ and $\operatorname{ESO}(\mathcal{Q})$ is, by hypothesis, closed under complementation, it follows that some prefix $Q^{\prime}$ such that $\left|Q^{\prime}\right|>1$ must belong to $\mathcal{Q}$; this is a contradiction. Hence, $\forall \notin \mathcal{Q}$, and consequently $\{\exists\} \subseteq \mathcal{Q} \subseteq\{\lambda, \exists\}$. Clearly, the complement $\overline{L_{1}}=\{a, b\}^{*} b\{a, b\}^{*}$ of the language $L_{1}$ in part $(i)$ of Proposition 11.1 is expressible in $\operatorname{ESO}(\exists)$, while $L_{1}$ is not; this is a contradiction. This proves $(i) \Rightarrow(i i)$.

$(i i) \Rightarrow(i i i) \quad$ Suppose that $\operatorname{ESO}(\mathcal{Q})$ captures REG, and assume that $\mathcal{Q} \cap \exists^{*} \forall\{\exists, \forall\}^{+}=\emptyset$. Then, since $\operatorname{ESO}(\mathcal{Q})$ is regular, from Theorem 8.7 it follows $\mathcal{Q} \subseteq \exists^{*} \forall$. By part $(i i)$ of Proposition 11.1, $a^{*} b^{*}$ is not expressible in $\operatorname{ESO}\left(\exists^{*} \forall\right)$; hence $\operatorname{ESO}(\mathcal{Q})$ does not capture REG, which is a contradiction.

$(i i i) \Rightarrow(i) \quad$ From Proposition 2.2 and the hypothesis, it follows that $\operatorname{ESO}(\mathcal{Q})$ captures REG; it is wellknown that REG is closed under complementation.

Corollary 11.5 $\operatorname{ESO}\left(\exists^{*} \forall\right)$ is the unique maximal regular ESO-prefix class which does not capture REG.

Note that Theorem 11.3, Corollary 11.5 and the results in [24] imply that model checking for regular ESO-prefix classes which do not capture REG is in low levels of $A C^{0}$.

From the dichotomy theorem for model checking (Theorem 9.3), we thus obtain the following dichotomy theorem for closure under complementation.

Theorem 11.6 (Dichotomy Theorem for Closure under Complementation) Let $\mathcal{Q} \subseteq\{\exists, \forall\}^{*}$ be any nontrivial prefix set. Then, under the assumption $\mathrm{NP} \neq \mathrm{co}-\mathrm{NP}$, the following statements are equivalent:

(i) $\operatorname{ESO}(\mathcal{Q})$ is closed under complementation.

(ii) $\operatorname{ESO}(\mathcal{Q})$ captures REG.

(iii) $\mathcal{Q} \cap \exists^{*} \forall\{\forall, \exists\}^{+} \neq \emptyset$ and $\mathcal{Q} \cap \exists^{*} \forall\left(\exists^{+} \forall\{\exists, \forall\}^{*} \cup \forall\{\exists, \forall\}^{+}\right)=\emptyset$. 


\section{Conclusion and Further Work}

In this paper, we have investigated the expressive power and complexity of the ESO-prefix classes over strings. We succeeded to settle some of the major question on this issue, giving a complete picture of the regular ESO-prefix classes, their relationship to MSO, the complexity of ESO prefix classes, and a characterization of those classes which are closed under complementation.

Some of the results were rather unexpected and required novel and involved combinatorial proof arguments. In particular, the fact that $\operatorname{ESO}\left(\exists^{*} \forall \exists^{*}\right)$, i.e., the ESO-standard prefix class in which the first-order parts of sentences are from the Ackermann class, is astonishing, and the proof of this nontrivial result appears to be rather difficult.

Our results show several dichotomy properties for ESO-prefix classes over strings. In particular, model checking for a class $\operatorname{ESO}(\mathcal{Q})$ is either feasible by a finite state automaton (and therefore in constant space), or it is NP-complete; a regular $\operatorname{ESO}(\mathcal{Q})$ is either equivalent to $\mathrm{MSO}$, or its expressive power is first-order (in fact, restricted to $\Sigma_{2}^{0}$ ); and, either $\operatorname{ESO}(\mathcal{Q})$ is not closed under complementation (assuming NP $\neq$ co-NP), or it captures the class REG of regular languages. In summary, with respect to any of these criteria, each ESO-prefix class $\operatorname{ESO}(\mathcal{Q})$ exhibits either a very good or an extremely bad behavior. Moreover, this matches observations on similar good/bad dichotomies of prefix classes which have been noted earlier in different contexts, e.g., 0-1 laws for ESO-prefix classes [30]. For a discussion, see Fagin's [16, p.20].

Three particular related research issues are currently under our investigation:

- The scope of the present paper are finite strings. However, infinite strings or $\omega$-words are another important area of research. In particular, Büchi has shown that an analogue of his theorem (Proposition 2.1) also holds for $\omega$-words [6]. For an overview of this and many other important results on $\omega$-words, we refer the reader to the excellent survey paper [51]. In this context, we have started to investigate which of the results of the present paper survive for $\omega$-words. For some results, such as Theorem 8.1 this is obviously the case since no finiteness assumption on the input word structures was made in the proof. For the generalization of other results, such as Theorem 7.1, further research is needed.

- We currently study which of our results survive in case a predefined linear order $<$ is available on the word structures, and in case the successor relation Succ is replaced by such a linear order. While for full ESO, Succ and < are freely interchangeable, because eiter predicate can be defined in terms of the other, this is not so for many of the limited ESO-prefix classes. However, preliminary results suggest that most of the results in this paper carry over to the $<$ case.

- A further issue is the extension of our results to the fragments $\Sigma_{k}^{1}(\mathcal{Q}), k>1$ of second-order logic, and to all of $\mathrm{SO}(\mathcal{Q})$.

Acknowledgements. The authors thank E. Grädel and E. Rosen for comments concerning decidability aspects of FO-prefix classes over finite strings; they proposed Corollary 10.3 and suggested a reduction from domino problems for proving the undecidability part. We also thank $\mathrm{H}$. Veith for useful comments.

\section{Appendix}

Theorem 8.1 Over strings, every $\operatorname{ESO}\left(\exists^{*} \forall \forall\right)$ sentence is equivalent to an MSO sentence. 
Proof. Let $\Phi$ be a formula $\exists \mathbf{R} \exists \mathbf{y} \forall x_{1}, x_{2} . \varphi$, where $\mathbf{y}=y_{1}, \ldots, y_{n}$ is a vector of FO variables and $\varphi$ is quantifier-free. Without loss of generality, we assume that $\min$ and $\max$ do not occur in $\varphi$ (they can be defined using additional variables $y_{\min }$ and $y_{\max }$ in $\mathbf{y}$ and adding $\neg \operatorname{Succ}\left(x_{1}, y_{\min }\right)$ and $\neg \operatorname{Succ}\left(y_{\max }, x_{1}\right)$ to $\varphi$ ).

Clearly, it suffices to consider only strings $W$ such that $|U(W)| \geq n+2$. Therefore, by a similar argument as in Lemma 8.4, we may assume without loss of generality that $\varphi$ is a DNF formula

$$
\varphi\left(\mathbf{y}, x_{1}, x_{2}\right)=\left(x_{1}=x_{2}\right) \vee \bigvee_{i, j}\left(x_{i}=y_{j}\right) \vee \bigvee_{\delta_{k}} \delta_{k},
$$

such that every clause $\delta_{k}$ is a complete type over $T=\left\{x_{1}, x_{2}, y_{1}, \ldots, y_{n}\right\}$ which includes for every pair $\xi, \chi$ of different variables in $T$ the literal $\xi \neq \chi$.

We show that all predicates in $R \in \mathbf{R}$ of arity $>1$ can be removed from $\Phi$ by introducing new monadic predicates $\mathbf{R}^{*}$.

First, we can easily remove all occurrences of atoms $R\left(\chi_{1}, \ldots, \chi_{m}\right)$ from $\varphi$ such that at most one of $x_{1}, x_{2}$ occurs in them as follows. Replace $R\left(\chi_{1}, \ldots, \chi_{m}\right)$,

- if $x_{1}$ occurs in it, by a unary atom $R^{\chi_{1}^{\prime}, \ldots, \chi_{m}^{\prime}}\left(x_{1}\right)$, where $\chi_{i}^{\prime}=*$ and if $\chi_{i}=x_{1}$ and $\chi_{i}^{\prime}=\chi_{i}$ otherwise, for $i=1, \ldots, m$;

- if $x_{2}$ occurs in it, by a unary atom $R^{\chi_{1}^{\prime}, \ldots, \chi_{m}^{\prime}}\left(x_{2}\right)$, where $\chi_{i}^{\prime}=*$ and if $\chi_{i}=x_{2}$ and $\chi_{i}^{\prime}=\chi_{i}$ otherwise, for $i=1, \ldots, m$;

- otherwise, by a nullary predicate $R^{\chi_{1}, \ldots, \chi_{m}}$.

For example, $R\left(y_{1}, x_{2}, y_{2}, x_{2}\right)$ is replaced by $R_{y_{1}, * y_{2}, *}\left(x_{2}\right)$, and $R\left(y_{1}, y_{3}\right)$ by $R_{y_{1}, y_{3}}\left(x_{1}\right)$. Intuitively, $R_{\chi_{1}^{\prime}, \ldots, \chi_{m}^{\prime}}\left(x_{i}\right)$ represents the atom $R\left(\chi_{1}, \ldots, \chi_{m}\right)\left[* / x_{i}\right]$.

Let $\mathbf{R}^{*}$ be the list of all predicates variables that we have introduced for all $R \in \mathbf{R}$ of arity $>1$, and let

$$
\Phi^{*}=\exists \mathbf{R}^{*} \exists \mathbf{R} \exists \mathbf{y} \forall x_{1}, x_{2} \cdot \varphi^{*},
$$

where $\varphi^{*}=\bigvee_{\delta \in \Delta(\Phi)} \delta^{*}$ and $\delta^{*}$ is the clause obtained from $\delta$ by the above replacements. Notice that in $\delta^{*}$, every literal with a predicate $R \in \mathbf{R}$ of arity $>1$ contains both $x_{1}$ and $x_{2}$.

It holds that over any string $W$ such that $|U(W)| \geq n+2, \Phi$ is equivalent to $\Phi^{*}$. To see this, observe that if $\Phi^{*}$ is true, then any interpreted atom $R^{\chi_{1}^{\prime}, \ldots, \chi_{m}^{\prime}}(e)$ occurring in some satisfied interpreted clause $\delta^{*}\left(\mathbf{y}, a_{1}, a_{2}\right)$ of $\varphi$, represents an interpreted atom $R\left(a_{1}, \ldots, a_{m}\right)$. By the inequality literals in $\delta^{*}\left(\mathbf{y}, a_{1}, a_{2}\right)$, different atoms $R^{\chi_{1}^{\prime}, \ldots, \chi_{m}^{\prime}}(e)$ occurring in satisfied clauses $\delta^{*}\left(\mathbf{y}, a_{1}, a_{2}\right)$ represent different $R$-atoms; moreover, no conflict between any $R$-atom represented by such an $R^{\chi_{1}^{\prime}, \ldots, \chi_{m}^{\prime}}(e)$ or by a nullary predicate $R^{\chi_{1}, \ldots, \chi_{m}}$ and any actual interpreted $R$-literal is present which occurs in some satisfied interpreted clause $\delta^{*}$.

It remains to remove all occurrences of atoms $R\left(\chi_{1}, \ldots, \chi_{m}\right)$ from $\Phi^{*}$ where $R \in \mathbf{R}$ and both $x_{1}, x_{2}$ occur among $\chi_{1}, \ldots, \chi_{m}$.

For each pair $\gamma^{*}, \delta^{*} \in \Delta\left(\Phi^{*}\right)$ such that the conjunction

$$
C_{\gamma^{*}, \delta^{*}}\left(\mathbf{y}, x_{1}, x_{2}\right)=\gamma^{*}\left(\mathbf{y}, x_{1}, x_{2}\right) \wedge \delta^{*}\left(\mathbf{y}, x_{2}, x_{1}\right)
$$

does not contain a pair of opposite literals, let $d_{\gamma^{*}, \delta^{*}}\left(\mathbf{y}, x_{1}, x_{2}\right)$ be the conjunction which results from $C$ after removal of all literals which involve a $R \in \mathbf{R}$ of arity $>1$; denote by $\Gamma$ the collection of all such $d_{\gamma^{*}, \delta^{*}}$. (Notice that for different $\gamma^{*}, \delta^{*}$, the clauses $d_{\gamma^{*}, \delta}$ are not necessarily different.) 
Let $\mathbf{R}^{1}$ be the monadic relations in $\mathbf{R}$, and let

$$
\Phi^{\prime}=\exists \mathbf{R}^{*} \mathbf{R}^{1} \exists \mathbf{y} \forall x_{1}, x_{2} \cdot \varphi^{\prime},
$$

where $\varphi^{\prime}=\bigvee_{\alpha \in \Gamma} \alpha\left(\mathbf{y}, x_{1}, x_{2}\right)$; notice that $\Phi^{\prime}$ is a monadic SO formula.

Claim 12.1 Over any string $W$ such that $|U(W)| \geq n+2, W \models \Phi^{\prime} \leftrightarrow \Phi^{*}$.

Hence, $W \models \Phi^{\prime} \leftrightarrow \Phi$, which proves the result.

To prove the claim, suppose first that $W \models \Phi^{*}$. Then, relations $\mathbf{R}^{*}, \mathbf{R}$ and a tuple $\mathbf{b}$ of elements in $W$ exists such that for every pair $a_{1}, a_{2}$ of elements in $W$, disjuncts $\gamma, \delta \in \Delta(\Phi)$ exist such that

$$
\left(W, \mathbf{R}^{*}, \mathbf{R}, \mathbf{b}\right) \models \gamma^{*}\left(\mathbf{b}, a_{1}, a_{2}\right) \wedge \delta^{*}\left(\mathbf{b}, a_{2}, a_{1}\right),
$$

and thus

$$
\left(W, \mathbf{R}^{*}, \mathbf{R}, \mathbf{b}\right) \models d_{\gamma^{*}, \delta^{*}}\left(\mathbf{b}, a_{1}, a_{2}\right),
$$

where $d_{\gamma^{*}, \delta^{*}} \in \Gamma$. This means $\left(W, \mathbf{R}^{*}, \mathbf{R}^{1}, \mathbf{b}\right) \models \forall x_{1}, x_{2} . \varphi^{\prime}$, and hence $W \models \Phi^{\prime}$.

Now suppose $W \models \Phi^{\prime}$. Then, for suitable relations $\mathbf{R}^{*}, \mathbf{R}^{1}$ on $W$ and a tuple $\mathbf{b}$ of elements in $W$, we have

$$
\left(W, \mathbf{R}^{*}, \mathbf{R}^{1}, \mathbf{b}\right) \models \forall x_{1}, x_{2} \cdot \varphi^{\prime},
$$

and thus for each pair $a_{1}, a_{2}$ of elements in $W$, there is some clause $d_{\gamma^{*}, \delta^{*}} \in \Gamma$, denoted by $\sigma_{a_{1}, a_{2}}$, such that $\left(W, \mathbf{R}^{*}, \mathbf{R}^{\prime}, \mathbf{b}\right) \models \sigma_{a_{1}, a_{2}}\left(a_{1}, a_{2}\right)$.

We define extensions for the $R \in \mathbf{R}$ of arity $>1$ as follows. For elements $a_{1}, a_{2} \in U(W)$ such that $a_{1} \leq a_{2}$ in $W$ according to Succ, we have $\sigma_{a_{1}, a_{2}}=d_{\gamma^{*}, \delta^{*}}$ for some $\gamma^{*}, \delta^{*} \in \Delta\left(\Phi^{*}\right)$. Let $C_{\gamma^{*}, \delta^{*}}\left(\mathbf{b}, x_{1}, x_{2}\right)$ be the conjunction from which $d_{\gamma^{*}, \delta^{*}}$ results in the construction of $\Phi^{\prime}$. Then, $C\left(\mathbf{b}, a_{1}, a_{2}\right)$ does not contain any pair of opposite literals. Include in $R$ every tuple $\left(e_{1}, \ldots, e_{m}\right)$ such that a positive literal $R\left(e_{1}, \ldots, e_{m}\right)$ occurs in $C\left(\mathbf{b}, a_{1}, a_{2}\right)$. Notice that if $\left(e_{1}, \ldots, e_{m}\right)$ is added to $R$, then there are no elements $a_{1}^{\prime}<a_{2}^{\prime}$ such that $a_{1}^{\prime} \neq a_{1}$ or $a_{2}^{\prime} \neq a_{2}$ for which $\neg R\left(e_{1}, \ldots, e_{m}\right)$ occurs in the satisfied conjunction $C_{\gamma^{*^{\prime}}, \delta^{*^{\prime}}}\left(\mathbf{b}, a_{1}^{\prime}, a_{2}^{\prime}\right)$, where $\sigma_{a_{1}^{\prime}, a_{2}^{\prime}}=d_{\gamma^{*^{\prime}}, \delta^{* \prime}}$, since the literals involving both $a_{1}^{\prime}$ and $a_{2}^{\prime}$ are different from those involving both $a_{1}$ and $a_{2}$. Then,

$$
\left(W, \mathbf{R}^{*}, \mathbf{R}, \mathbf{b}\right) \models \gamma^{*}\left(\mathbf{b}, a_{1}, a_{2}\right) \wedge \delta^{*}\left(\mathbf{b}, a_{2}, a_{1}\right) ;
$$

consequently,

$$
\left(W, \mathbf{R}^{*}, \mathbf{R}, \mathbf{b}\right) \models \forall x_{1}, x_{2} \bigvee_{\delta^{*} \in \Delta\left(\Phi^{*}\right)} \delta^{*}\left(\mathbf{b}, x_{1}, x_{2}\right),
$$

which means $W \models \Phi^{*}$. This proves the equivalence of $\Phi^{*}$ and $\Phi^{\prime}$ over $W$ and concludes the proof of the theorem.

\section{References}

[1] S. Abiteboul, R. Hull, and V. Vianu. Foundations of Databases. Addison-Wesley, 1995.

[2] M. Ajtai. $\Sigma_{1}^{1}$ formulae on finite structures. Annals of Pure and Applied Logic, 24:1-48, 1983.

[3] D. Basin and N. Klarlund Hardware verification using monadic second-order logic. Proc. 7th Intl. Conf. on Computer Aided Verification (CAV'95), LNCS 939, pp. 31-41, 1995. 
[4] J. Berstel. Transductions and Context-Free Languages. Teubner, Stuttgart, 1979.

[5] E. Börger, E. Grädel, and Y. Gurevich. The Classical Decision Problem. Springer, Berlin Heidelberg, 1997.

[6] J. R. Büchi. On a Decision Method in Restricted Second-Order Arithmetic. In E. Nagel et al. (eds), Proc. International Congress on Logic, Methodology and Philosophy of Science, pages 1-11, Stanford, CA, 1960. Stanford University Press.

[7] J. R. Büchi. Weak second-order arithmetic and finite automata. Z. Math. Logik Grundlagen Math., 6:66-92, 1960.

[8] M. de Rougemont. Second Order and Inductive Definability on Finite Structures. Zeitschrift für Mathematische Logik und Grundlagen der Mathematik, 33:47-63, 1987.

[9] A. Durand, C. Lautemann, and T. Schwentick. Fragments of Binary NP. Journal of Logic and Computation, 1998 (to appear). TR Nr. 1/96, Johannes Gutenberg-Universität Mainz, Germany.

[10] H.-D. Ebbinghaus and J. Flum. Finite Model Theory. Perspectives in Mathematical Logic. Springer, 1995.

[11] S. Eilenberg. Automata, Languages, and Machines. Academic Press, New York, 1974.

[12] T. Eiter and G. Gottlob. On the Expressiveness of Frame Satisfiability and Fragments of Second Order Logic. Journal of Symbolic Logic, 1998 (to appear). Technical Report CD-TR 96/92, Institut für Informationssysteme, TU Wien, 1996.

[13] T. Eiter, G. Gottlob, and Y. Gurevich. Normal Forms for Second-Order Logic over Finite Structures, and Classification of NP Optimization Problems. Annals of Pure and Applied Logic, 78:111-125, 1996.

[14] R. Fagin. Generalized First-Order Spectra and Polynomial-Time Recognizable Sets. In R. M. Karp, editor, Complexity of Computation, pages 43-74. AMS, 1974.

[15] R. Fagin. Generalized Monadic Spectra. Zeitschrift für Mathematische Logik und Grundlagen der Mathematik, 21:89-96, 1975.

[16] R. Fagin. Finite-model theory—a personal perspective. Theoretical Computer Science, 116(1):3-31, 1993.

[17] E. Grädel. The Expressive Power of Second-Order Horn Logic. In Proceedings STACS-91, LNCS 480, pages 466-477, 1991.

[18] E. Grädel. Capturing Complexity Classes with Fragments of Second Order Logic. Theoretical Computer Science, 101:35-57, 1992.

[19] E. Grandjean. Universal quantifiers and time complexity of random access machines. Mathematical Systems Theory, 13:171-187, 1985.

[20] Y. Gurevich. Logic and the Challenge of Computer Science. In E. Börger, editor, Trends in Theoretical Computer Science, chapter 1. Computer Science Press, 1988.

[21] J.G. Henriksen, J. Jensen, M. Jørgensen, N. Klarlund, B. Paige, T. Rauhe, and A. Sandholm Mona: Monadic Second-order Logic in Practice. in Proceedings of Tools and Algorithms for the Construction and Analysis of Systems, First Intl. Workshop, TACAS'95, Springer LNCS 1019, pp. 89-110, 1996.

[22] J. Hopcroft and J. Ullman. Introduction to Automata Theory, Languages, and Computation. Addison-Wesley, Reading, MA, 1979.

[23] N. Immerman. Relational Queries Computable in Polynomial Time. Information and Control, 68:86-104, 1986.

[24] N. Immerman. Languages that Capture Complexity Classes. SIAM Journal of Computing, 16:760-778, 1987.

[25] N. Immerman. Descriptive Complexity Theory. Springer, 1998 (to appear). 
[26] N. Klarlund. Mona \& Fido: The Logic-Automaton Connection in Practice. In Proc. Conference on Computer Science Logic (CSL '97), LNCS. Springer, 1998 (to appear).

[27] P. Kolaitis and C. Papadimitriou. Some Computational Aspects of Circumscription. Journal of the ACM, 37(1):1$15,1990$.

[28] P. Kolaitis and C. H. Papadimitriou. Why Not Negation By Fixpoint? Journal of Computer and System Sciences, 43:125-144, 1991.

[29] P. Kolaitis and M. N. Thakur. Approximation Properties of NP Minimization Classes. Journal of Computer and System Sciences, 50:391-411, 1995.

[30] P. Kolaitis and M. Y. Vardi. 0-1 Laws and Decision Problems for Fragments of Second-Order Logic. Information and Computation, 87:302-338, 1990.

[31] C. Lautemann, T. Schwentick, and D. Thérien. Logics for context-free languages. In Proc. 1994 Annual Conference of the EACSL, pages 205-216, 1995.

[32] D. Leivant. Descriptive Characterizations of Computational Complexity. Journal of Computer and System Sciences, 39:51-83, 1989.

[33] J. F. Lynch. The quantifier structure of sentences that characterize nondeterministic time complexity. Computational Complexity, 2:40-66, 1992.

[34] R. McNaughton and S. Papert. Counter-Free Automata. MIT Press, Cambridge, Massachusetts, 1971.

[35] F. Olive. A Conjunctive Logical Characterization of Nondeterministic Linear Time. In Proc. Conference on Computer Science Logic (CSL '97), LNCS. Springer, 1998 (to appear).

[36] L. Pacholski and W. Szwast. On the 0-1Law for the Existential Second-Orde Minimal Gödel Sentences With Equality. In Proceedings Sixth Annual Symposium on Logic in computer Science LICS-91, pages 290-285. IEEE Computer Science Press, 1991.

[37] A. Panconesi and D. Ranjan. Quantifiers and Approximation. Theoretical Computer Science, 107:145-163, 1993.

[38] C. H. Papadimitriou and M. Yannakakis. Optimization, Approximation, and Complexity Classes. Journal of Computer and System Sciences, 43:425-440, 1991.

[39] J.-E. Pin. Varieties of Formal Languages. North Oxford, London and Plenum, New York, 1986.

[40] J.-E. Pin. Logic On Words. Bulletin of the EATCS, 54:145-165, 1994.

[41] J.-E. Pin. Semigroups and Automata on Words. Annals of Mathematics and Artificial Intelligence, 16:343-384, 1996.

[42] E. Rosen. An Existential Fragment of Second Order Logic. Revised manuscript, December 1996.

[43] D. Saccá. The Expressive Powers of Stable Models for Bound and Unbound DATALOG Queries. Journal of Computer and System Sciences, 54(3):441-464, 1997.

[44] T. J. Schaefer. The Complexity of Satisfiability Problems. In Proceedings of the Tenth ACM Symposium on the Theory of Computing (STOC-78), pages 216-226, San Diego, California, 1978.

[45] J. Schlipf. The expressive powers of logic programming semantics. Journal of Computer and System Sciences, 51(1):64-86, 1995. Abstract in Proc. PODS 90, pp. 196-204.

[46] T. Schwentick. Graph Connectivity and Monadic NP. In Proceedings IEEE FOCS '94, pages 614-622, 1994.

[47] T. Schwentick. Graph Connectivity, Monadic NP and Built-in Relations of Moderate Degree. In Proceedings 22nd International Colloquium on Automata, Languages, and Programming (ICALP '95), pages 405-416, 1995. 
[48] L. J. Stockmeyer. The Polynomial-Time Hierarchy. Theoretical Computer Science, 3:1-22, 1977.

[49] H. Straubing. Finite Automata, Formal Logic, and Circuit Complexity. Birkhäuser, Berlin, Boston, Basel, 1994.

[50] H. Straubing, D. Thérien, and W. Thomas. Regular Languages Defined with Generalized Quantifiers. Information and Computation, 118:289-301, 1995.

[51] W. Thomas. Automata on Infinite Objects. In J. van Leeuwen, editor, Handbook of Theoretical Computer Science, volume B, chapter 4. Elsevier Science Publishers B.V. (North-Holland), 1990.

[52] W. Thomas. Languages, Automata, and Logic. In G. Rozenberg and A. Salomaa, editors, Handbook of Formal Language Theory, volume III, pages 389-455. Springer, 1996.

[53] B. A. Trakhtenbrot. Finite Automata and the Logic of Monadic Predicates. Dokl. Akad. Nauk SSSR, 140:326$329,1961$.

[54] M. Vardi. Complexity of Relational Query Languages. In Proceedings 14th STOC, pages 137-146, San Francisco, 1982. 